Cochrane Database of Systematic Reviews

\title{
Surgical treatment for tubal disease in women due to undergo in vitro fertilisation (Review)
}

Johnson N, van Voorst S, Sowter MC, Strandell A, Mol BWJ

Johnson N, van Voorst S, Sowter MC, Strandell A, Mol BWJ.

Surgical treatment for tubal disease in women due to undergo in vitro fertilisation.

Cochrane Database of Systematic Reviews 2010, Issue 1. Art. No.: CD002125.

DOI: 10.1002/14651858.CD002125.pub3.

www.cochranelibrary.com 
TABLE OF CONTENTS 1

ABSTRACT

PLAIN LANGUAGE SUMMARY

BACKGROUND

OBJECTIVES

METHODS

RESULTS

Figure 1.

Figure 2.

Figure 3.

Figure 4.

Figure 5.

Figure 6.

DISCUSSION

AUTHORS' CONCLUSIONS

ACKNOWLEDGEMENTS

REFERENCES

CHARACTERISTICS OF STUDIES

DATA AND ANALYSES

Analysis 1.1. Comparison 1 Laparoscopic surgery on the fallopian tube (all types) VERSUS No surgery on the fallopian tube (all types), Outcome 1 Ongoing pregnancy rate.

Analysis 1.2. Comparison 1 Laparoscopic surgery on the fallopian tube (all types) VERSUS No surgery on the fallopian tube (all types), Outcome 2 Clinical pregnancy rate.

Analysis 1.3. Comparison 1 Laparoscopic surgery on the fallopian tube (all types) VERSUS No surgery on the fallopian tube (all types), Outcome 3 Pregnancy rate - any definition.

Analysis 1.4. Comparison 1 Laparoscopic surgery on the fallopian tube (all types) VERSUS No surgery on the fallopian tube (all types), Outcome 4 Ectopic pregnancy rate.

Analysis 1.5. Comparison 1 Laparoscopic surgery on the fallopian tube (all types) VERSUS No surgery on the fallopian tube (all types), Outcome 5 Miscarriage rate.

Analysis 1.6. Comparison 1 Laparoscopic surgery on the fallopian tube (all types) VERSUS No surgery on the fallopian tube (all types), Outcome 6 Surgical complication rate.

Analysis 2.1. Comparison 2 Occlusion of the fallopian tube VERSUS no intervention on the fallopian tube, Outcome 1 Ongoing pregnancy rate.

Analysis 2.2. Comparison 2 Occlusion of the fallopian tube VERSUS no intervention on the fallopian tube, Outcome 2 Clinical pregnancy rate.

Analysis 2.3. Comparison 2 Occlusion of the fallopian tube VERSUS no intervention on the fallopian tube, Outcome 3 Pregnancy rate - any definition.

Analysis 2.4. Comparison 2 Occlusion of the fallopian tube VERSUS no intervention on the fallopian tube, Outcome 4 Ectopic pregnancy rate.

Analysis 2.5. Comparison 2 Occlusion of the fallopian tube VERSUS no intervention on the fallopian tube, Outcome 5 Miscarriage rate.

Analysis 3.1. Comparison 3 Aspiration of the hydrosalpinges versus non aspiration of hydrosalpinges, Outcome 1 Clinical pregnancy rate.

Analysis 3.2. Comparison 3 Aspiration of the hydrosalpinges versus non aspiration of hydrosalpinges, Outcome 2 Biochemical pregnancy rate.

Analysis 3.3. Comparison 3 Aspiration of the hydrosalpinges versus non aspiration of hydrosalpinges, Outcome 3 Pregnancy rate - any definition.

Analysis 3.4. Comparison 3 Aspiration of the hydrosalpinges versus non aspiration of hydrosalpinges, Outcome 4 Ectopic pregnancy rate.

Analysis 3.5. Comparison 3 Aspiration of the hydrosalpinges versus non aspiration of hydrosalpinges, Outcome 5 Miscarriage rate.

Analysis 3.6. Comparison 3 Aspiration of the hydrosalpinges versus non aspiration of hydrosalpinges, Outcome 6 Surgical complication rate. 
Analysis 4.1. Comparison 4 Laparoscopic surgery on the fallopian tube (all types) VERSUS (any other) laparoscopic surgery on the fallopian tube, Outcome 1 Ongoing pregnancy rate.

Analysis 4.2. Comparison 4 Laparoscopic surgery on the fallopian tube (all types) VERSUS (any other) laparoscopic surgery on the fallopian tube, Outcome 2 Clinical pregnancy rate.

Analysis 4.3. Comparison 4 Laparoscopic surgery on the fallopian tube (all types) VERSUS (any other) laparoscopic surgery on the fallopian tube, Outcome 3 Pregnancy rate - any definition.

Analysis 4.4. Comparison 4 Laparoscopic surgery on the fallopian tube (all types) VERSUS (any other) laparoscopic surgery on the fallopian tube, Outcome 4 Ectopic pregnancy rate.

Analysis 4.5. Comparison 4 Laparoscopic surgery on the fallopian tube (all types) VERSUS (any other) laparoscopic surgery on the fallopian tube, Outcome 5 Miscarriage rate.

Analysis 5.1. Comparison 5 Surgical treatment (all types) VERSUS no surgical treatment, Outcome 1 Salpingectomy (all methods) VERSUS no surgical treatment.

Analysis 5.2. Comparison 5 Surgical treatment (all types) VERSUS no surgical treatment, Outcome 2 Tubal occlusion (all methods) VERSUS no surgical treatment.

Analysis 5.3. Comparison 5 Surgical treatment (all types) VERSUS no surgical treatment, Outcome 3 Aspiration of hydro salpingeal fluid (all methods) VERSUS no surgical treatment.

Analysis 6.1. Comparison 6 Laparoscopic surgery on the fallopian tube (all types) VERSUS (any other) laparoscopic surgery on the fallopian tube, Outcome 1 Tubal occlusion (all methods) VERSUS Salpingectomy (all methods).

\section{APPENDICES}

WHAT'S NEW

HISTORY

CONTRIBUTIONS OF AUTHORS

DECLARATIONS OF INTEREST

SOURCES OF SUPPORT

DIFFERENCES BETWEEN PROTOCOL AND REVIEW

INDEX TERMS 
[Intervention Review]

\section{Surgical treatment for tubal disease in women due to undergo in vitro fertilisation}

Neil Johnson ${ }^{1}$, Sabine van Voorst ${ }^{2}$, Martin C Sowter ${ }^{3}$, Annika Strandell ${ }^{4}$, Ben Willem J Mol ${ }^{5}$

1Department of Obstetrics \& Gynaecology, University of Auckland, Auckland, New Zealand. 2Faculty of Medicine, University of Maastricht, Maastricht, Netherlands. ${ }^{3}$ Fertility Plus, Auckand Hospital, Auckland, New Zealand. ${ }^{4}$ Obstetrics and Gynecology, University of Gothenburg, Kungälv, Sweden. 50bstetrics and Gynaecology, Máxima Medical Center, Veldhoven, Netherlands

Contact address: Neil Johnson, Department of Obstetrics \& Gynaecology, University of Auckland, PO Box 92019, Auckland, 1003, New Zealand.n.johnson@auckland.ac.nz.

Editorial group: Cochrane Gynaecology and Fertility Group.

Publication status and date: Edited (no change to conclusions), published in Issue 1, 2010.

Citation: Johnson N, van Voorst S, Sowter MC, Strandell A, Mol BWJ. Surgical treatment for tubal disease in women due to undergo in vitro fertilisation. Cochrane Database of Systematic Reviews 2010, Issue 1. Art. No.: CD002125. DOI: 10.1002/14651858.CD002125.pub3.

Copyright @ 2010 The Cochrane Collaboration. Published by John Wiley \& Sons, Ltd.

\section{A B S T R A C T}

\section{Background}

Tubal disease, and particularly hydrosalpinx, has a detrimental effect on the outcome of in-vitro fertilisation (IVF). Performing a surgical intervention such as salpingectomy, tubal occlusion, aspiration of the hydrosalpinx fluid, or salpingostomy, prior to the IVF procedure in women with hydrosalpinges is thought improve the likelihood of successful outcome.

\section{Objectives}

To assess and compare the value of surgical treatments for tubal disease prior to IVF.

\section{Search methods}

Trials were sought in the Cochrane Menstrual Disorders and Subfertility Group trials register, the Cochrane Central Register of Controlled Trials (CENTRAL), MEDLINE, EMBASE, PSYCHMED and in Conference proceedings and reference lists up until Ocober 282009 . Researchers in the field were contacted to reveal unpublished studies.

\section{Selection criteria}

All trials comparing a surgical treatment for tubal disease with a control group generated by randomisation were considered for inclusion in the review.

\section{Data collection and analysis}

Two reviewers independently assessed trial quality and extracted data. The studied outcomes were live birth, ongoing pregnancy, viable-, clinical- and biochemical pregnancy, ectopic pregnancy, miscarriage, multiple pregnancy, ovarian function and complications.

\section{Main results}

Five randomised controlled trials involving 646 women were included in this review. Four studies assessed salpingectomy versus no treatment, two of which also included a tubal occlusion arm, and one trial assessed aspiration versus no treatment. No trials reported on the primary outcome: live birth. The odds of ongoing pregnancy (Peto OR 2.14,95\% $\mathrm{Cl} 1.23$ to 3.73) and of clinical pregnancy (Peto OR 2.31, $95 \% \mathrm{Cl} 1.48$ to 3.62 ) however were increased with laparoscopic salpingectomy for hydrosalpinges prior to IVF. Laparoscopic occlusion of the fallopian tube versus no intervention did not increase the odds of ongoing pregnancy significantly (Peto $\mathrm{OR} 7.24,95 \% \mathrm{Cl} 0.87$ to 59.57 ) but the odds of clinical pregnancy (Peto OR 4.66, 95\% Cl 2.47 to 10.01) had sufficient power to show a significant increase. Comparison of tubal occlusion to salpingectomy did not show a significant advantage of either surgical procedure in terms of ongoing pregnancy (Peto OR: 
$1.65,95 \% \mathrm{Cl} 0.74,3.71$ ) or clinical pregnancy (Peto OR $1.28,95 \% \mathrm{Cl} 0,76$ to 2.14 ). One RCT reported efficacy of ultrasound guided aspiration, however the odds of pregnancy did not show a significant increase in the odds of clinical pregnancy (Peto OR 1.97, 95\% $\mathrm{Cl} 0.62$ to 6.29 ), and confidence intervals were wide. Throughout the different comparisons no significant differences were seen in adverse effects of surgical treatments.

\section{Authors' conclusions}

Surgical treatment should be considered for all women with hydrosalpinges prior to IVF treatment. Previous evidence supported only unilateral salpingectomy for a unilateral hydrosalpinx (bilateral salpingectomy for bilateral hydrosalpinges). This review now provides evidence that laparoscopic tubal occlusion is an alternative to laparoscopic salpingectomy in improving IVF pregnancy rates in women with hydrosalpinges. Further research is required to assess the value of aspiration of hydrosalpinges prior to or during IVF procedures and also the value of tubal restorative surgery as an alternative (or as a preliminary) to IVF.

\section{PLAIN LANGUAGE SUMMARY}

\section{Surgical treatment for tubal disease in women due to undergo in vitro fertilisation}

Diseases of the fallopian tube, such as hydrosalpinx can severely reduce the chances of pregnancy from in vitro fertilisation (IVF). Removing (salpingectomy) or occluding blocked or diseased fallopian tubes before in IVF can increase pregnancy and live birth rates for women on the IVF program. This review of trials found both laparoscopic salpingectomy and tubal occlusion prior to IVF increase the odds of pregnancy. However, other treatment options still need to be evaluated and the place of restorative tubal surgery in clinical practice needs to be evaluated. 


\section{B A C K G R O U N D}

Accounting for $14 \%$ of female infertility (RCOG); tubal pathology ranks among the most frequent causes of subfertility (Evers 2002). A spectrum of severity is recognized at laparoscopy, ranging from peritubal adhesions, through damaged fimbriae or distorted tubal anatomy, tubal blockage to the most severe manifestation of tubal disease: hydrosalpinx.

The couple with tubal factor subfertility has two therapeutic options to overcome the mechanical obstructions present in tubal disease: in-vitro fertilization (IVF) or reconstructive surgery. The place of reconstructive surgery is a topic of debate, as selection of patients and the method for tubal surgery is challenging (Dechaud 2004). IVF was primarily developed to treat tubal infertility (Steptoe 1978) and has been shown to be effective. However, a paradox emerged after recognition that IVF in patients with tubal disease was associated with lower implantation rates and an increased risk of early pregnancy loss (Andersen 1994; Camus 1999; Englert 1987; Strandell 1994; Vandromme 1995; and Zeyneloglu 1998) than after IVF in other subfertile patients. This deleterious effect of tubal disease on IVF outcome may be related to the severity of tubal damage (Csemiczky 1996; Vasquez 1995). Dissapointingly, the treatment was less effective in the patient group it was designed for than for subfertile patients with other causes for their subfertility.

There are several theories to explain the underlying mechanism by which hydrosalpinges exert a deleterious effect on IVF outcome. In these theories hydrosalpinx fluid seems to have a key role (Strandell 2002). It has been proposed that the hydrosalpinx fluid may affect the transferred embryo; possibly by embryotoxic factors (Mukherjee 1996) or a common factor deleterious to embryonic development and possibly by the lack of nutrients (Dickens 1995 and Tay 1997). The bathing of the endometrial cavity in hydrosalpinx fluid may interfere with the endometrial interaction with the transferred embryo necessary for implantation (Akman 1996; Fleming 1996; Freeman 1996; Katz 1996; Meyer 1997; Strandell 1994 ). Furthermore mechanical effects exerted by hydrorrhoea (the leakage of hydrosalpinx fluid through the uterine cavity) (Andersen 1996; Bloechle 1997; Mansour 1991), the presence of a thin layer of fluid upon the endometrial surface (Andersen 1996; Sharara 1999) and changes in endometrial peristalsis by the fluid (Eytan 2001) may wash-out or hinder implantation of the transferred embryo. Furthermore it has been postulated that hydrosalpinges, during IVF stimulation, may exert a negative influence on oocytes in early follicular recruitment (Freeman 1996).

The lowered efficacy of IVF alone led to the concept that fallopian tube surgery prior to IVF might improve results. Suggested surgical interventions are: salpingectomy (Dechaud 1998, Strandell 1999) salpingostomy (Goldstein 1998), aspiration of hydrosalpinx fluid (Aboulghar 1990; Russel 1991; Sowter 1997; Van Voorhis 1998) tubal ligation (Murray 1998) and tubal occlusion by means of Filshie clips (Darwish 2005,2006, 2007), Essure micro-inserts (Kerin 2005; Rosenfield 2005) or electrocautery (Murray 1998; Stadtmauer 2000; Surrey 2001).

Prior to the first version of this review in 2004, there was a shift in clinical practice towards surgical intervention prior to IVF. The majority of the clinicians in the field was convinced of efficacy by mostly retrospective or non-randomised published studies. Prior to this review there was a wide variation amongst surgeons in the surgical methods performed (Johnson2002).
Despite guidelines recommending salpingectomy, based on the evidence provided by the previous version of this review, there remained a wide variation in the methods offered and employed in surgical management, according to a French (Ducarme 2006) and an English (Hammadieh 2004) survey. Salpingectomy remains the most frequently undertaken procedure. This is still justified, as the previous version of this review analysed data on salpingectomy and the efficacy of other surgical treatments had not been proven by sufficiently powered prospective randomised controlled trials.

Each treatment has its own merits and drawbacks. Salpingectomy has the advantage that chronically infected tissue is removed in total; removing the risk of abscess formation or torsion and increasing the accessibility of the ovary during oocyte retrieval in IVF (Kontoravdis 2006). Drawbacks however are the invasiveness of the procedure itself and the difficulty of the procedure in case of dense adhesions. Furthermore it has been suggested that salpingectomy may affect ovarian function by interfering with ovarian blood flow (Dar 2000; Lass 1998). There are however reassuring data to suggest that ovarian compromise does not occur after salpingectomy (Strandell 2001). Other possible adverse effects are interstitial pregnancy; which has been reported in two cases (Herman 1991; Shariff 1994) or ovarian pregnancy (Hsu 2005). Formation of cornual fistulae (Hsu 2005) and cornual rupture have been described (Inovay 1999) in single cases after salpingectomy by electrocautery. Salpingectomy is the treatment with the most permanent character - any possibility of conceiving spontaneously is removed. This is a psychological burden for the patient and an important drawback as many gynaecologists are aware of women who conceived spontaneously after being deemed to have hopeless tubal infertility. Compared to salpingectomy, aspiration, salpingostomy and tubal occlusion are thought to have the advantage of being less invasive, safer and easier to perform in the case of dense adhesions (Stadtmauer 2000), with shorter hospital stays (Surrey 2001; Taylor 2001). Tubal occlusion is also associated with risks of tubal surgery. A case of adnexal torsion ( $\mathrm{La}$ Combe 2003) has been reported after occlusion by electrocautery. Theoretically aspiration may cause a flare-up of infection. The main difference of these procedures with salpingectomy is the less permanent character; this is regarded to be an advantage; however at the same time this is a disadvantage because a hydrosalpinx may recur (Bloechle 1997; Van Voorhis 1998; Sowter 1997) after the intervention.

It is a major decision for patients who have suffered from infertility to undergo a surgical procedure to remove fallopian tubes prior to IVF and surgical procedures are not without risks. Therefore it is important to have the best available evidence that these interventions are beneficial.

\section{O B JECTIVES}

To assess the efficacy of surgical treatment of tubal disease prior to IVF. The following surgical treatments for tubal disease were considered: salpingectomy (both unilateral and bilateral), tubal occlusion (both unilateral and bilateral), salpingostomy and hydrosalpinx fluid aspiration. The hypothesis that surgical treatment of tubal disease prior to IVF is beneficial by increasing the pregnancy and live birth rate, without substantially increasing complications related to the intervention, was tested. 


\section{METHODS}

\section{Criteria for considering studies for this review}

\section{Types of studies}

All randomised controlled trials (RCTs) comparing women undergoing surgical treatment for tubal disease prior to IVF with a control group (which could include another type of surgery, medical treatment or no treatment) were considered eligible for inclusion.

\section{Types of participants}

Inclusion criteria: women with diagnosed tubal disease due to undergo IVF

Exclusion criteria: none

\section{Types of interventions}

All studies comparing salpingectomy, tubal occlusion, salpingostomy, tubal occlusion, ultrasound guided aspiration of hydrosalpinx fluid or any other surgical procedure performed uni and/or bilaterally for tubal pathology; with no intervention or with any other mentioned intervention, were considered eligible for inclusion.

\section{Types of outcome measures}

\section{Primary outcomes}

(1) Live birth rate - defined as number of live birth deliveries per woman randomised.

\section{Secondary outcomes}

(1) Pregnancy

(a) Ongoing pregnancy rate- defined as number of pregnancies surpassing the first twelve weeks of pregnancy, trimester, per woman randomised

(b) Viable pregnancy rate - rate of pregnancy diagnosed by gestational sac with fetal heart activity on ultrasound.

(c) Clinical pregnancy rate - rate of pregnancy diagnosed by gestational sac on ultrasound and biochemical pregnancy test.

(d) Biochemical pregnancy rate - rate of pregnancy diagnosed by biochemical pregnancy test.

(e) Pregnancy rate according to any definition - 'pregnancy rate' undefined.

(2) Adverse effects

(a) Ectopic pregnancy rate. - rate of ectopic pregnancies per woman randomised.

(b) Miscarriage rate - rate of miscarried pregnancies, per woman randomised.

(c) Multiple pregnancy rate - rate of multiple pregnancies amongst clinical pregnancies

(d) Surgical complication rate. - rate of any complication of the interventions considered.

(e) Ovarian response to IVF - defined as number of oocytes retrieved.

If outcomes were not reported as above, sufficient information had to be available to convert results to the outcomes stated above.

\section{Search methods for identification of studies}

Eligible studies since the previous version of this review in 2004 were identified by a predefined search strategy in electronic databases, handsearching, reference lists and contacting authors.

\section{Electronic searches}

A predefined and customised search developed by the trial search coordinator of the Menstrual Disorders and Subfertility Group was employed in the following databases:

- Menstrual Disorders and Subfertility Group Specialised Register (inception to 28.10.09)

- Ovid The Cochrane Central Register of Controlled Trials (CENTRAL) (from inception to $4^{\text {th }}$ Quarter 2009)

- Ovid MEDLINE (1950 to 28.10.09)

- Ovid EMBASE (01.01.08 to 28.10.09).

The detailed search strategy is presented in Appendix 1

\section{Searching other resources}

Relevant conference proceedings were retrieved by handsearching proceedings of the European Society for Human Reproduction, the British Fertility Society, the Fertility Society of Australia, the American Society of Reproductive Medicine, the World Congress on In Vitro Fertilisation and Human Reproductive Genetics, ESGE, ANZJOG and in ISI Proceedings.

Reference lists of included trials, eligible studies, conference abstracts and relevant review articles were searched for more potentially relevant studies. Furthermore, the authors of previously included and excluded trials, and the first or corresponding author of eligible trials were contacted to ascertain if they were aware of any ongoing or unpublished trials.

\section{Data collection and analysis}

\section{Selection of studies}

Eligibility of identified citations, according to the previously stated criteria, was assessed by two reviewers independently. This was done by reading the abstract and if necessary by retrieving the article in full. If reviewers disagreed about eligibility, a third reviewer was to be contacted (MS) to achieve consensus on eligibility.

\section{Data extraction and management}

Two reviewers (NJ and SVV) independently assessed study characteristics and methodological details of included studies using data extraction forms. Differences in opinion were to be resolved by consultation of a third reviewer (MS). Where additional information on trial methodology or original trial data was required corresponding authors were contacted. Reminder correspondence was sent if a reply was not received within two weeks.

A detailed list of the items assessed during data extraction is presented in Appendix 2.

\section{Assessment of risk of bias in included studies}

Two reviewers (NJ and SVV) independently assessed study characteristics and methodological details of included studies using data extraction forms. Differences in opinion were to be 
resolved by consultation of a third reviewer (MS). Where additional information on trial methodology or original trial data was required corresponding authors were contacted. Reminder correspondence was sent if a reply was not received within two weeks.

A detailed list of the items assessed during data extraction is presented in Appendix 2.

\section{Measures of treatment effect}

As this review considers dichotomous data (pregnant or not pregnant after IVF with or without surgical intervention), data were expressed as a Peto odds ratio (OR) with $95 \%$ confidence intervals (Cl).

\section{Unit of analysis issues}

As defined by the in- and exclusion criteria, this review only incorporated randomised controlled trials. Therefore different levels of randomisation did not have to be taken into account in the analysis: the numbers of observations matched the numbers of people randomised by definition.

\section{Dealing with missing data}

Missing data were analysed intention to treat: the total number or randomised participants were used as the denominator, irrespective of how the original study authors analysed the data. Missing data, and how this was handled by the trial, was also incorporated as a quality item. Based on the extent to which data were missing, the potential impact of the missing data on the results was to be explored by sensitivity analysis.

\section{Assessment of heterogeneity}

Prior to meta-analysis, statistical heterogeneity between the results of different studies was examined graphically by inspecting the scatter in the data points on the graphs and the overlap in their confidence intervals and, more formally, by checking the results of chi-squared tests.

\section{Assessment of reporting biases}

Regarding the source of data; a funnel plot was planned to examine the possibility of a publication bias if there were ten or more included trials. When funnel plots suggested liability of a publication bias; funnel plots were to be attached.

\section{Data synthesis}

Statistical analysis was performed in accordance with the guidelines for statistical analysis of the Cochrane handbook.

Peto odds ratios (OR) were combined for meta-analysis with RevMan 5 software using the Peto-modified Mantel-Haenszel method, where pooling was acceptable in the absence of heterogeneity. An increase in the odds of a particular outcome (which may be beneficial, for example in the case of live birth, or detrimental, for example in the case of ectopic pregnancy) was displayed graphically in the meta-analyses to the right of the centre-line and a decrease in the odds of an outcome was displayed graphically to the left of the centre-line.

\section{Subgroup analysis and investigation of heterogeneity}

If heterogeneity was present clinical considerations were examined which theoretically could lead to heterogeneity. Further investigation was to be done by sensitivity analysis.

\section{Sensitivity analysis}

A sensitivity analysis was planned to examine the stability of the results in relation to potential risk of bias and the source of the data (published or unpublished).

\section{RE S U L T S}

\section{Description of studies}

\section{Results of the search}

The employed search strategy identified 103 potentially relevant citations. Contacting authors did not reveal any unpublished trials. Nine of the 103 citations were potentially eligible and retrieved in full for final in or exclusion:Darwish 2005,2006, 2007; Gelbaya 2006; Hammadieh 2008; Hotineanu 2007; Kontoravdis 2006; Moshin 2006 and Zolghadri 2006. Previously, four studies were found to be eligible in this review (Dechaud 1998; Goldstein 1998; Mardesic 1999; and Strandell 1999).

\section{Included studies}

Previously three RCTs, with a total of 295 women, met the criteria for inclusion in the review (Dechaud 1998; Goldstein 1998; Strandell 1999). Of the newly identified trials Hammadieh 2008; Kontoravdis 2006 and Moshin 2006 were included.

Inclusion of Goldstein 1998 was reconsidered as it was unsure whether randomisation was performed; it appeared that patients may have been their own historical controls. This study was presented only in abstract form and has not been published in a peer reviewed journal. Attempts to contact the author to clarify this were unsuccessful and the trial was therefore excluded. This review therefore considers five trials, analysing a total of 646 women.

Of the included trials, two trials assessed the effectiveness of laparoscopic salpingectomy compared to no salpingectomy for women with hydrosalpinges prior to IVF (Dechaud 1998; Strandell 1999); two trials assessed and compared effectiveness of laparoscopic tubal occlusion with laparoscopic salpingectomy and no intervention (Kontoravdis 2006, Moshin 2006); Hammadieh 2008 was the only trial to contribute to the comparison of ultrasound guided aspiration of the hydrosalpinges during the IVF cycle with no aspiration.

\section{Design:}

All studies were randomised controlled trials. Time of follow-up was longest in Dechaud 1998; which had a follow-up time of 1 to 5 years in which data on outcomes of more than one IVF attempt per patient were collected. Follow-up of the four other studies (Hammadieh 2008; Kontoravdis 2006; Moshin 2006; Strandell 1999) was up to the results of the first IVF cycle. The follow-up scheme was known for all trials.

\section{Sample size}

Dechaud 1998 and Hammadieh 2008 were the trials with the smallest study size, each assessing around 60 women. Kontoravdis 2006 reported results of 112 women; these women were randomised into three intervention groups. Moshin 2006 and 
Strandell 1999 were the largest trial both reporting results of 204 women; Moshin 2006 also had three randomisation groups.

Losses to follow-up and withdrawal occurred, but were of acceptable size in Strandell $1999(\mathrm{~N}=17)$; and Kontoravdis 2006 $(\mathrm{N}=3)$, and dealt with by intention to treat. In the trials of Moshin 2006 and Hammadieh 2008 there were no losses to follow-up or withdrawals; in the trial of Dechaud 1998 loss to follow-up and withdrawal was not reported, but the number of analysable results was the same as the number of women randomised.

Three of the five trials (Dechaud 1998; Hammadieh 2008; Moshin 2006) were performed in single centre studies; 2 of these settings were academic, one was non-academic. Strandell 1999; was a multicenter trial (9 Nordic centres) and Kontoravdis 2006 was a trial performed in an academic- and a non-academic centre. None of the studies reported data on the characteristics of the surgeons involved.

\section{Participants:}

Inherent to RCT's, participants were recruited prospectively; correspondence clarified that patient sampling was consecutive in the trials of Kontoravdis 2006 and Moshin 2006. The method of participant sampling was not reported in the remaining studies.

The important prognostic factor of age was stated by all trials; all participants were at least below the age of 41 . The population in the trail of Moshin 2006 was younger; as age $\leqq 35$ was applied as an inclusion criterion. The duration of subfertility was stated in two trials; the duration varied from 22 to 89 months in the trial of Dechaud 1998 and after application of the inclusion criterion (subfertility $>2$ years) the duration of subfertility varied from 19 to 73 months in the trial of Moshin 2006. Distribution was similar among intervention and control group(s) in both trials. The proportion of women with primary and secondary subfertility was specified in Strandell 1999; Dechaud 1998; Hammadieh 2008; Kontoravdis 2006 and Moshin 2006 (after correspondence). The majority of patients had primary subfertility; the distribution of primary subfertility throughout the intervention groups was similar. A standard fertility investigative work-up was done in two trials: Dechaud 1998 and Moshin 2006. Kontoravdis 2006 and Hammadieh 2008 only reported baseline FSH's. Hammadieh 2008; Kontoravdis 2006 and Strandell 1999; included couples with coexistent male factor infertility undergoing ICSI; the proportion of these couples were similar in groups within the studies. As Strandell 1999 was a multicentre trial; it was specified that ICSI patients were included only when the unit had an ICSI program with success rates equivalent to conventional IVF for non-male factor causes of infertility. Kontoravdis 2006; also a multicentre trial, did not specify this. No trials stated previous fertility treatments; all trials either specified that patients did not undergo IVF treatment prior to the trial or applied this as an exclusion criterion.

The extent of the cause of infertility was defined in all trials. In the Dechaud 1998 trial, tubal infertility was defined by both radiologic and laparoscopic criteria as being severe and unsuitable for tubal repair. The inclusion criteria for this trial were either (1) a hysterosalpingogram showing extensive inflammatory disease in the proximal part of tube with diverticula extending to involve more than two centimetres of the tubal isthmus or hydrosalpinges with poor prognosis because of disturbed mucosal folds or irregular walls; or (2) a laparoscopy revealing the presence of proximal nodes or an inflammatory and thick-walled hydrosalpinx.
The inclusion criteria for the Strandell 1999 trial were unior bi-lateral hydrosalpinges, defined as a distally occluded or pathologically dilated fallopian tube or one which became so on diagnostic tubal patency testing. The Hammadieh 2008 trial included women with uni- or bi-lateral hydrosalpinx diagnosed by ultrasound during ovarian stimulation in the IVF cycle or previous ultrasound examination. Furthermore, this trial included women with hydrosalpinges from the waiting list for tubal surgery. The Kontoravdis 2006 trial included women with unilateral of bilateral hydrosalpinges; confirmed by HSG, suitability for IVFICSI treatment. Moshin 2006 included women with bilateral distal tubal occlusion diagnosed by HSG and hydrosalpinges visible on ultrasound. The distribution of tubal pathology uni- and bilaterally was specified in trials of Hammadieh 2008; Kontoravdis 2006; and Strandell 1999. Dechaud 1998. In the trial of Moshin 2006 all patients had bilateral tubal pathology. The proportion of hydrosalpinges visible on ultrasound was reported in Hammadieh 2008; Moshin 2006; Kontoravdis 2006 and Strandell 1999; . Besides in the trial of Hammadieh 2008, where patients were included after diagnosis of hydrosalpinges by ultrasound, it was not clear whether identified subgroups were pre-specified.

The IVF protocol was clearly described in all trials. In the trial of Moshin 2006 higher dosages of gonadotropins were used than in other trials (225 IU). Except for Strandell 1999 and Dechaud 1998, all studies reported results over 1 IVF cycle. It was possible to extract results over the first cycle separate from further IVF cycles. Timing of IVF after the intervention was reported by all studies. Of the trials assessing efficacy of salpingectomy or laparoscopic occlusion, Kontoravdis 2006; Moshin 2006 and Strandell 1999 had a time interval from surgery to IVF of approximately 2 to 3 months. The trial of Dechaud 1998 had a wider variation in time from intervention to IVF; varying from 1 to 17 months. The time interval between randomisation and IVF was similar in control groups compared to intervention groups in the trials of Hammadieh 2008 and Dechaud 1998, differed slightly in the trial of Strandell 1999 and was not specified in the trials of Kontoravdis 2006 and Moshin 2006.

\section{Interventions}

In the previous version of this review salpingectomy was the only surgical intervention in the included trials. Trials did differ in whether uni or bilateral salpingectomy was performed; the cointerventions which were performed; and in the control group to which they were compared.

Dechaud 1998 assessed laparoscopic bilateral salpingectomy regardless of whether tubal disease was uni- or bi-lateral; Strandell 1999 assessed uni- or bi-lateral salpingectomy (depending on whether uni- or bi-lateral hydrosalpinges were present). In fact, of the 116 women randomised to laparoscopic surgery, there were 63 bilateral salpingectomies, 40 unilateral salpingectomies, six proximal ligations with distal fenestrations, one salpingostomy, one adhesiolysis, one no intervention as the fallopian tubes were found to be patent and five women did not undergo surgery these were all included in the treatment group in an intention to treat analysis. The control groups differed among the previously included studies. In the trials of Dechaud 1998 and Strandell 1999; no salpingectomy was performed. In the trial of Dechaud 1998 patients did undergo adhesiolysis. Kontoravdis 2006; Moshin 2006 also contributed data to the comparison of salpingectomy with no salpingectomy. In the trial of Kontoravdis 2006 salpingectomy was performed uni- or bilaterally depending on the presence of 
either uni- or bilateral tubal pathology. In the trial Moshin 2006 the intervention was performed bilaterally. Control groups were similar namely: no intervention. No co-interventions were performed in Strandell 1999 and Kontoravdis 2006; this was unclear for the trial of Moshin 2006. It was deemed appropriate to pool the data from these four trials for meta-analysis since they all prospectively evaluated a group of women who underwent salpingectomy on the fallopian tube (salpingectomy in most cases in all three trials) versus a control group who did not have this surgical intervention.

There was only one trial available to compare aspiration of hydrosalpinges to no aspiration. Hammadieh 2008 assessed aspiration of hydrosalpinges under ultrasound guidance; immediately after oocyte retrieval during the IVF cycle. This was performed uni- or bilaterally depending on the distribution of the hydrosalpinges. To prevent infection in the intervention group, co interventions administered were: using two different aspiration needles in case of bilateral tubal pathology, and intravenous Augmentin was administered at the time of intervention followed by 3 days of oral Azithromycin.

There were two trials evaluating proximal tubal occlusion (Kontoravdis 2006; Moshin 2006). In the trial of Kontoravdis 2006 tubal occlusion was performed by bipolar diathermy at two separate sites on the isthmic segment of the tube. This was performed uni- or bilaterally depending on the presence respectively of uni or bilateral tubal pathology. In the trial of Moshin 2006 tubal occlusion was accomplished laparoscopically by clipping of the tubes proximally. Both studies had similar control groups: no intervention in women with hydrosalpinges. As both studies had two comparison groups (salpingectomy besides a nonintervention group) data of the control group were also used in the assessment of salpingectomy in our review. No information on the performance of co-interventions was given in Moshin 2006. The fact that the method and approach of occlusion was different in the trial of Moshin 2006 was dealt with by classifying Moshin 2006 under an own subgroup within the outcome. Because the studies of Moshin 2006 and Kontoravdis 2006 both assessed occlusion; the main mechanism of the treatment was similar pooling was thought to be appropriate. The trials of Moshin 2006 and Kontoravdis 2006 were pooled and no statistical heterogeneity was present.

\section{Outcome}

None of the trials reported live birth rates strictly. Where live birth rates were supposedly reported they were combined with ongoing pregnancy rates (Strandell 1999). As live births are inherently also ongoing pregnancies, Strandell 1999 was pooled with ongoing pregnancy outcomes for meta-analysis.

Dechaud 1998 and Kontoravdis 2006 reported ongoing pregnancy rates as defined in our objectives. Hammadieh 2008 and Moshin 2006 did not report the ongoing pregnancy.

The number of pregnancies was reported in all trials. The trials of Hammadieh 2008; Kontoravdis 2006; Moshin 2006 and Strandell 1999 defined clinical pregnancy as the presence of a gestational sac on ultrasound; Kontoravdis 2006 also looked at the presence of a fetal pole. Correspondence clarified that Moshin 2006 also looked at cardiac activity of pregnancies at 6 weeks (which would reflect viable pregnancy); it is unclear whether results reflect only numbers with positive cardiac activity and therefore purely viable pregnancies, or gestational sacs and viable pregnancies combined. Therefore results were classified as clinical pregnancies. Hammadieh 2008 presented results of biochemical pregnancy. No studies presented outcomes of IVF as viable pregnancies. Dechaud 1998 was the only study not to define 'pregnancy' as a viable, clinical or biochemical pregnancy; these results were therefore only classified under 'pregnancy of any kind.

Except for Dechaud 1998; all data of ongoing and clinical pregnancy were reported either per IVF cycle per woman or sufficient data was available to be converted to pregnancy per cycle per woman. Dechaud 1998 reported the pregnancy rate for the first cycle of IVF after recruitment and for all IVF cycles - the rates per woman could be extracted from these data. Cumulative pregnancy rates were also presented although these could not be used in this meta-analysis owing to assumptions made by the authors about the couples for whom no data were available. Data of only the first cycle could be extracted. There has been a subsequent publication of the cumulative results from the Nordic multicenter trial in 2001 (Strandell 1999). Results have been reported on an actual treatment received basis (rather than intention-to-treat in the original publication), justified on the grounds that 24 out of 77 women who were randomised to no surgical intervention, subsequently underwent salpingectomy after one or two failed cycles before proceeding with further IVF, the results of these cycles then being included in the analysis. These results have been excluded from the meta-analysis. The intention-to-treat data, requested from the trial authors, have also been excluded from the meta-analysis owing to a potential for bias from the number of women randomised to no intervention who have now, in fact, received the surgical intervention. The original 1999 data (Strandell 1999) have been maintained in the meta-analysis.

Ectopic pregnancy rates per woman (the number of women experiencing at least one ectopic pregnancy over the time period) could be extracted from all trials except for Moshin 2006; which did not report these results. All studies reporting ectopic pregnancy rates, except for Kontoravdis 2006 did not define ectopic pregnancy.

Data on miscarriage rates could be extracted from all trials. The trial of Hammadieh 2008 reported miscarriage rates per biochemical pregnancy; after correspondence it was possible to convert this data to miscarriage rate per clinical pregnancy. All other studies did not define miscarriage rate as to whether this was per clinical pregnancy per woman. Therefore we extracted data for miscarriage rates where possible; but these rates were not pooled in the metaanalysis.

Surgical complication rates were reported by the trials of Hammadieh 2008 and Strandell 1999.

IVF-treatment characteristics, and mean number of retrieved oocytes were stated or clarified after correspondence by all trials. They were similar between intervention and control groups.

The included studies and their methodological details are summarised comprehensively in the Characteristics of included studies table.

\section{Excluded studies}

Previously, the Mardesic 1999 trial, was excluded because the control group was historical (the same women prior to their salpingectomy). Furthermore no pregnancies occurred in the historical control group prior to salpingectomy. 
According to the abstracts of the potentially relevant citations, Gelbaya 2006 and Zolghadri 2006 were excluded after reading the full report of the trial. The Gelbaya 2006 trial did not meet inclusion criteria as the trial appeared to be a retrospective non randomised trial. The trial of Zolghadri 2006 was excluded because IVF was not performed. The objective of the trial was to assess continuation of spontaneous pregnancy after tubal surgery.

The Characteristics of excluded studies table summarises the reasons for exclusion.

Identified non-randomised studies (Aboulghar 1990; Savic 1999;Sowter 1997 Stadtmauer 2000; Van Voorhis 1998) were previously summarised in a non-randomised studies table. The current employed search has identified an additional retrospective trial (Gelbaya 2006) however as the identification of nonrandomised studies was not a predefined objective and a filter for RCT's was applied in our search strategy, the search results may not be representative. This and the fact that the number of randomised studies has increased was reason to no longer present a table of non-randomised studies.

\section{Risk of bias in included studies}

Blinding of physician performing IVF and the outcome assessor was not performed in most trials (Dechaud 1998; Hammadieh 2008; Kontoravdis 2006; Strandell 1999) or not reported to be performed (Moshin 2006).

Incomplete data was addressed with an intention to treat analysis in Strandell 1999 and Kontoravdis 2006 (clarified after correspondence) and seemed to be performed in Hammadieh 2008; and Moshin 2006; since number of randomised patients was the same as the number of analysed patients. Length of follow-up was long enough to adequately detect stated outcomes. The source of funding was either stated or clarified after correspondence in all trials except Dechaud 1998.

A power calculation was performed in four studies (Dechaud 1998; Kontoravdis 2006; Strandell 1999; Hammadieh 2008); but sufficient sample size was not achieved in three of them and the calculation was not performed correctly in one trial . Strandell 1999 powered the trial based on 300 couples, but stopped recruiting after 204 couples had been recruited and randomised, owing to inability to reach the target number within a reasonable time as a result of an apparent decrease in the incidence of hydrosalpinx in Scandinavia during the study period. In the trial of Dechaud 1998 the inability to generate the large number of patients for the study led to early cessation of the trial. In the trial of Kontoravdis 2006 it may be questioned whether the power calculation was adhered to because large differences were anticipated, which were unlikely to be achieved. Hammadieh 2008 stated that the sample size was not attained after four years because patients preferred salpingectomy rather than aspiration. Although three trials (Strandell 1999; Dechaud 1998; Hammadieh 2008) were stopped early, there is no suggestion that this was related to their accruing outcomes.

It was uncertain whether a power calculation was performed in Moshin 2006, but as study sizes were small it may be presumed that they have not been adhered to if they where performed.

Funnelplots were inspected; no publication bias is apparent.

Methodological quality assessment is summarised graphically in Figure 1 and Figure 2.

Figure 1. Methodological quality graph: review authors' judgements about each methodological quality item presented as percentages across all included studies.

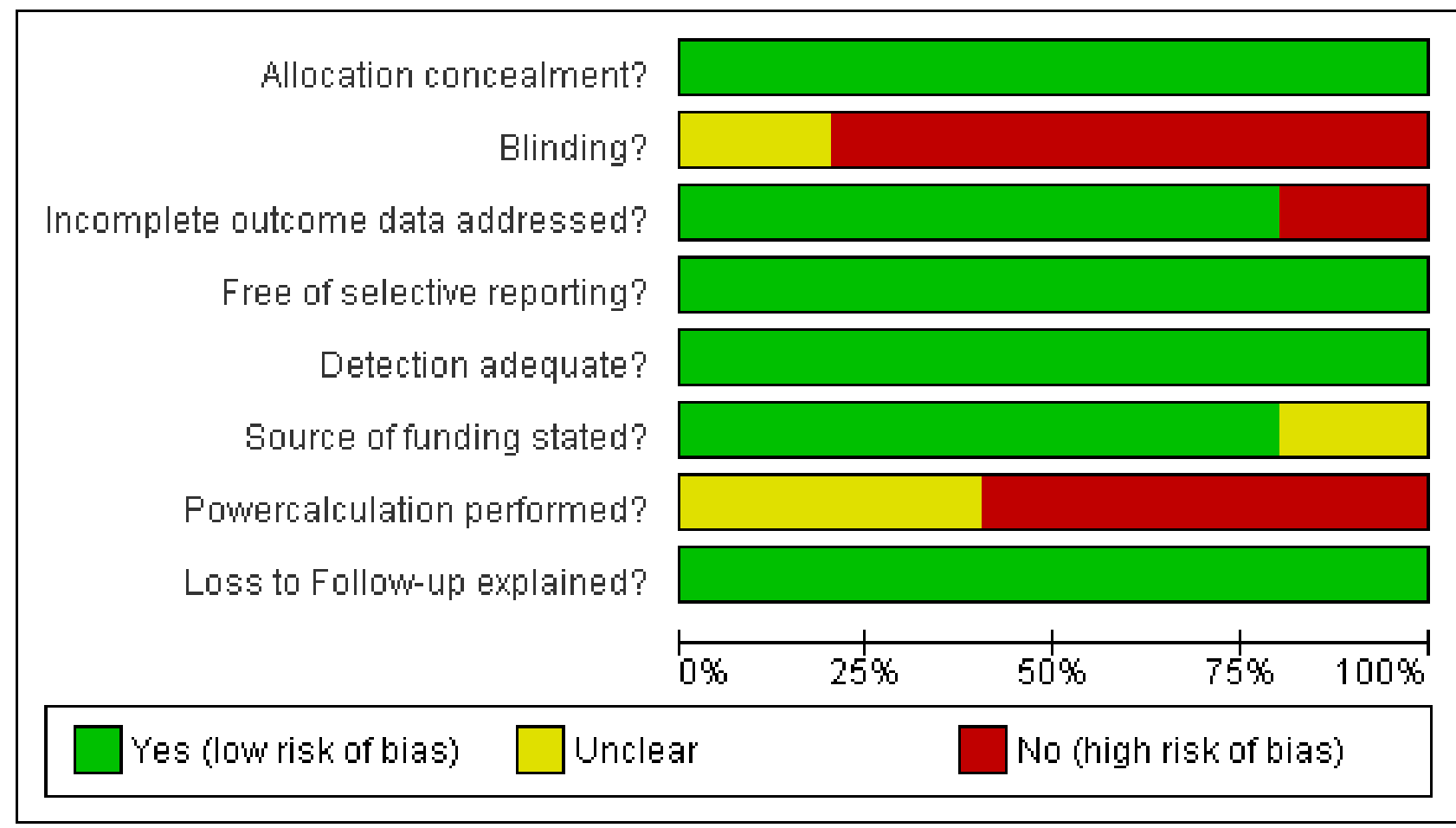

Surgical treatment for tubal disease in women due to undergo in vitro fertilisation (Review)

Copyright $\odot 2010$ The Cochrane Collaboration. Published by John Wiley \& Sons, Ltd. 
Figure 2. Methodological quality summary: review authors' judgements about each methodological quality item for each included study.

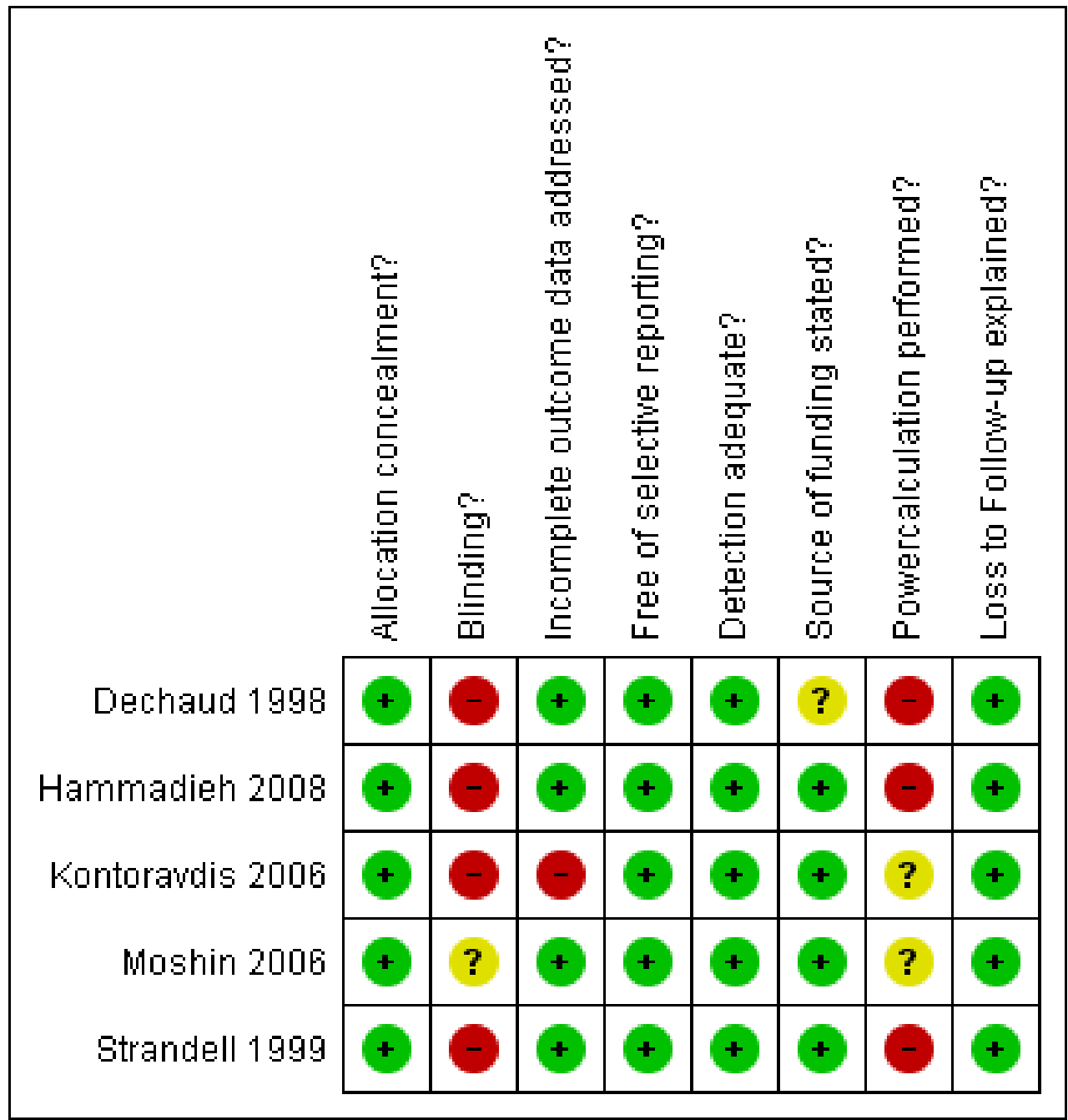

\section{Effects of interventions}

Surgical treatment for hydrosalpinges by laparoscopic salpingectomy versus non-surgical management significantly increased the odds of ongoing pregnancy (Peto OR 2.20, 95\% Cl 1.26 to $3.82 ; 329$ women, three trials, Figure 3), and of clinical pregnancy (Peto OR 2.31, 95\% Cl 1.48 to $3.62 ; 395$ women, three trials, Figure 3 ), and pregnancy according to any definition (Peto OR $2.49,95 \% \mathrm{Cl}$ 1.60 to 3.86 ; 455 women, three trials, Figure 3). 
Figure 3. Forest plot of comparison: Surgical treatment (all types) VERSUS no surgical treatment: Salpingectomy (all methods) VERSUS no surgical treatment. Outcomes: Ongoing pregnancy rate, Clinical pregnancy rate, Pregnancy rate according to any definition, Ectopic pregnancy rate, Miscarriage rate, Surgical complication rate.

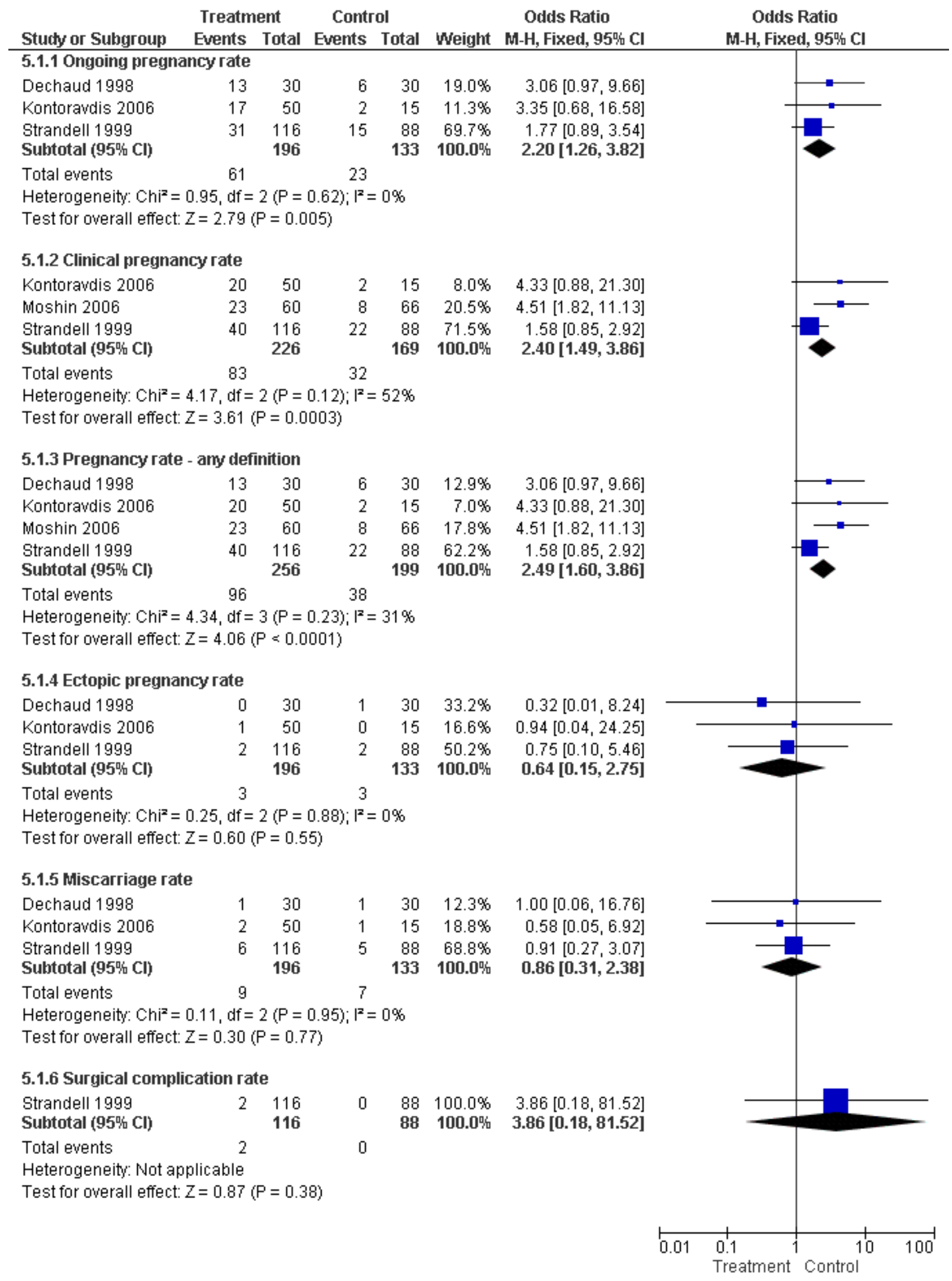


Surgical treatment by laparoscopic occlusion of the fallopian tube versus no intervention on the fallopian tube did not increase the odds of ongoing pregnancy (Peto OR $7.21,95 \% \mathrm{Cl} 0.87$ to 59.57; 65 women, one trial,Figure 4 ) significantly, but the observation had lack of power and therefore very wide confidence intervals. However the odds of clinical pregnancy were increased significantly (Peto OR 4.66, 95\% Cl 2.17 to $10.01 ; 209$ women, two trials, Figure 4) where meta-analysis of women had improved power.

Figure 4. Forest plot of comparison: Surgical treatment (all types) VERSUS no surgical treatment: Tubal occlusion (all methods) VERSUS no surgical treatment. Outcomes: Ongoing pregnancy rate, Clinical pregnancy rate, Ectopic pregnancy rate, Miscarriage rate, Surgical complication rate.

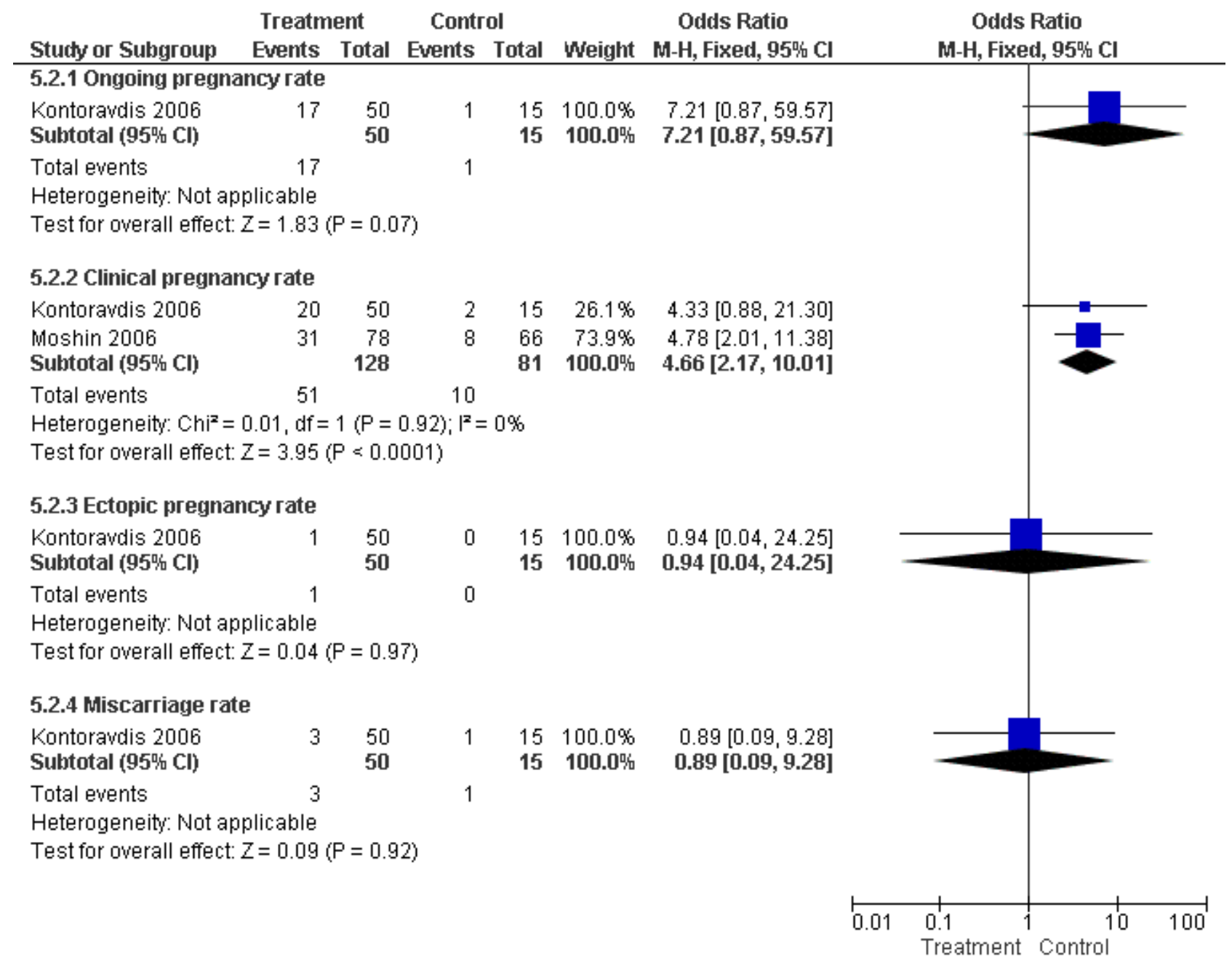

The one trial reporting on aspiration does not report a significantly increased odds of clinical pregnancy (Peto OR $1.97,95 \% \mathrm{Cl} 0.62$ to 6.29; 64 women, one trial, Figure 5) and of biochemical pregnancy
(Peto OR 2.78, 95\% 0.93 to $8.27,64$ women, one trial, Figure 5). No data were available on live birth or ongoing pregnancy rate. 
Figure 5. Forest plot of comparison: Surgical treatment (all types) VERSUS no surgical treatment: Aspiration of hydro salpingeal fluid (all methods) VERSUS no surgical treatment. Outcomes: Ongoing pregnancy rate, Clinical pregnancy rate, Ectopic pregnancy rate, Miscarriage rate, Surgical complication rate.

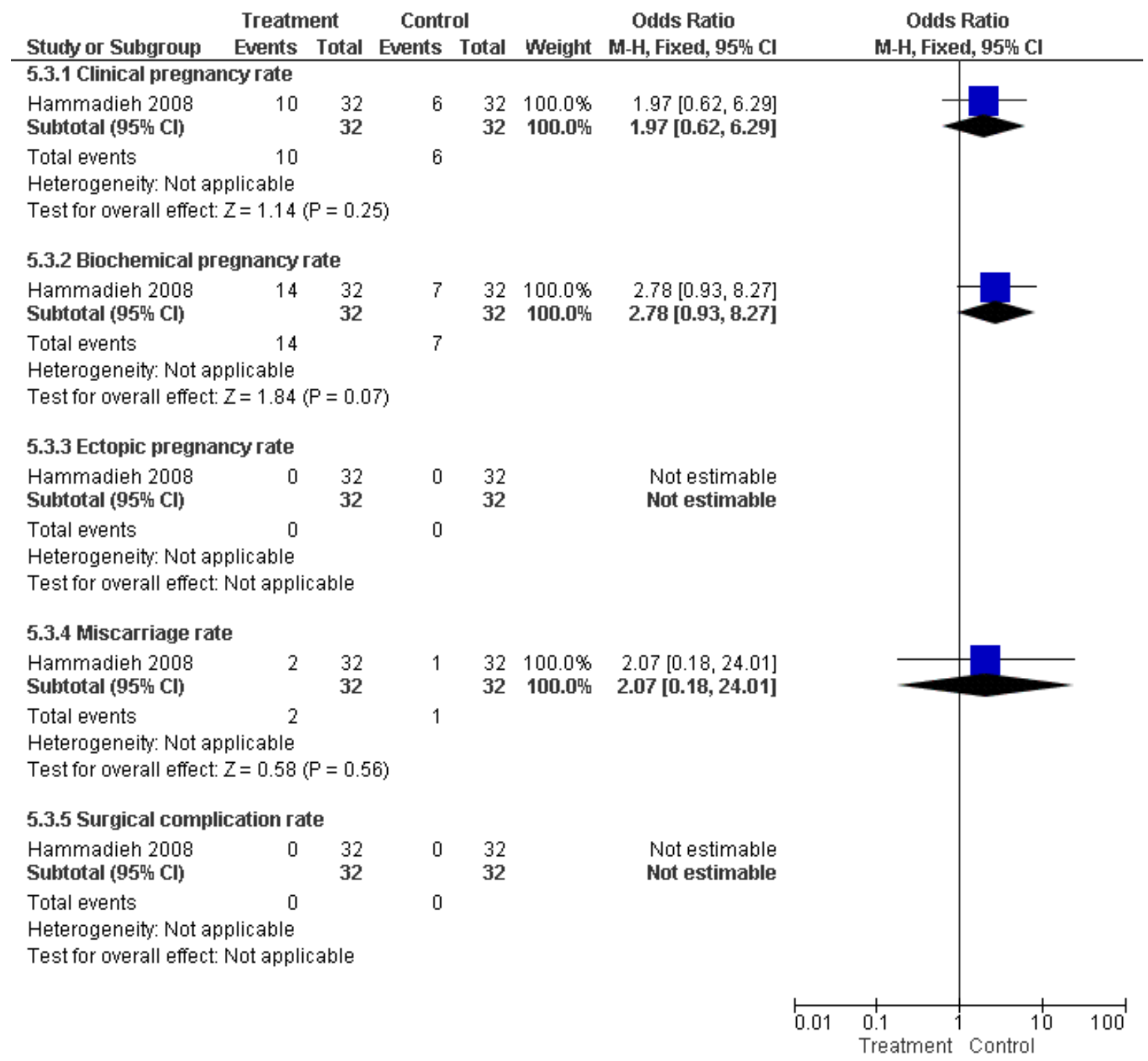

Comparison of laparoscopic salpingectomy and laparoscopic occlusion did not show a significant difference in terms of ongoing pregnancy based on 1 study (Peto OR $1.65,95 \% \mathrm{Cl} 0.74$ to 3.71 ;
100 women, one trial,Figure 6 ), clinical pregnancy (Peto OR 1.28, $95 \% \mathrm{Cl} 0,76$ to $2.14 ; 238$ women, two trials, Figure 6). No data were available on live birth or ongoing pregnancy rate. 
Figure 6. Forest plot of comparison: Laparoscopic surgery on the fallopian tube (all types) VERSUS (any other) laparoscopic surgery on the fallopian tube: Tubal occlusion (all methods) VERSUS Salpingectomy (all methods). Outcomes: Ongoing pregnancy rate, Clinical pregnancy rate, Ectopic pregnancy rate, Miscarriage rate.

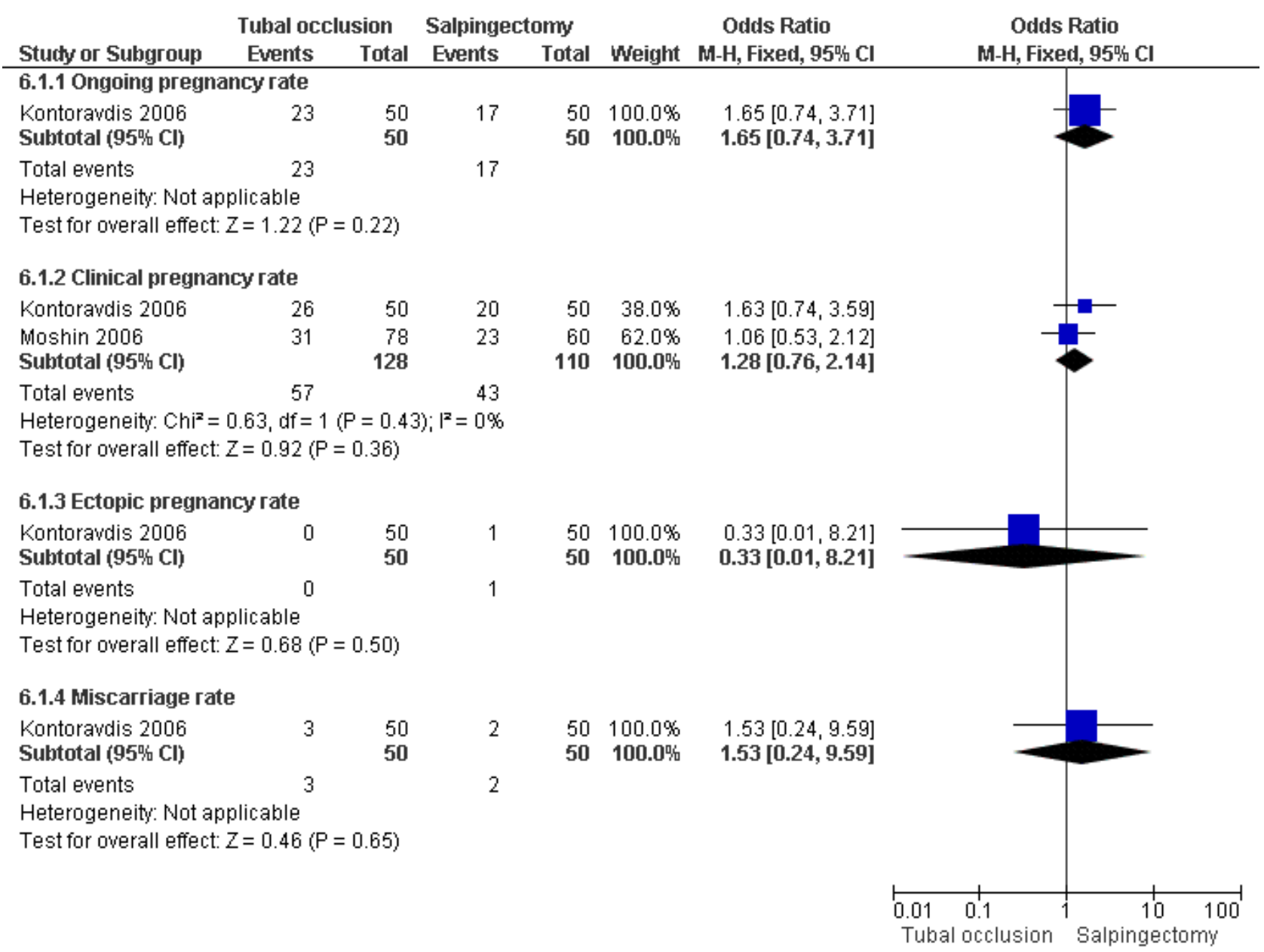

Throughout the different comparisons no significant differences were seen in the odds of ectopic pregnancy, miscarriage, and the odds of surgical treatment complications (Figure 3; Figure 4; Figure 5; Figure 6).The trial of Hammadieh 2008 did not report a significant difference in infection rate between intervention and control group. Strandell 1999 reported two surgical complications, one conversion from laparoscopic surgery to laparotomy and, one case of a postoperative infection.

The mean number of oocytes (SD) retrieved in patient groups treated with salpingectomy (Strandell 199910.6( \pm 5.9 ); Dechaud 1998 10.1( \pm 5.0$)$; Kontoravdis 200612.1( \pm 5.0$)$; Moshin 2006 $10.4( \pm 6.0))$ did not differ with the mean number of oocytes retrieved in control groups $(10.6( \pm 6.1)$ Strandell 1999; 10.6( \pm 6.1$)$ Dechaud $1998 ; 10.9( \pm 5.1)$ Kontoravdis 2006; 9.8 $( \pm 5.5)$ Moshin 2006) nor with number of oocytes retrieved after other surgical interventions for hydrosalpinges: (aspiration: 14.1 $( \pm 7.1)$ Hammadieh 2008 ; tubal occlusion: 11.6( \pm 4.9$)$ Kontoravdis 2006; 10.2( \pm 5.7$)$ Moshin 2006).

Generally there was no heterogeneity present across trials. There was a moderate heterogeneity present in the comparison group of salpingectomy where Strandell 1999 and Moshin 2006 were pooled, for the outcome of clinical pregnancy $(12=52 \%)$ (Analysis
5.1) and a mild heterogeneity for the outcome of any pregnancy (12=31\%)(Analysis 1.3); chi2results for heterogeneity however were not significant. Pooling was further justified by the fact that no heterogeneity was present where these trials where pooled elsewhere and by clinical characteristics of trials.

\section{DISCUSSION}

\section{Summary of main results}

This Cochrane review summarises the current evidence on the effectiveness of tubal surgery prior to IVF.

The effectiveness of laparoscopic salpingectomy, that was already demonstrated in the previous version of this review, is supported more strongly by the current data, in terms of ongoing pregnancy and clinical pregnancy. Unfortunately, a complete report on live birth as an outcome was lacking in all of the included studies. The ongoing pregnancy rates in the intervention and control group were $31 \%$ and $17.6 \%$.

Of other surgical interventions, laparoscopic tubal occlusion is shown to improve the odds of clinical pregnancy after metaanalysis for the first time. Results for the outcome ongoing 
pregnancy reported by only one trial were not significant (but this comparison was underpowered). The beneficial effect of laparoscopic tubal occlusion is further supported by the finding that comparison of laparoscopic occlusion and laparoscopic salpingectomy showed similar efficacy.

There is only one randomised controlled trial reporting on aspiration. This study was underpowered leaving the need for more trials assessing aspiration of hydrosalpinges.

Currently, no conclusions can be made regarding the adverse effects of interventions. Data on ectopic pregnancy, miscarriage, and or surgical complications of treatment remain underpowered.

\section{Overall completeness and applicability of evidence}

This Cochrane review was performed according to current standards of the Cochrane collaboration and of the Menstrual Disorders and Subfertility group.

Applicability and completeness of this review reflects the study characteristics and completeness by which these were reported. Besides the lack of reporting on the primary outcome (live birth), reported outcomes, patient and treatment characteristics were reported quite complete. Given that the interventions would not be expected to alter the occurrence of pregnancy loss from second trimester onwards, ongoing pregnancy presents an entirely suitable surrogate primary outcome for live birth.

Current findings apply for women that were recruited by included studies: women $<41$ years of age with hydrosalpinges, diagnosed by diagnostic laparoscopy and/or HSG, who have not had IVF previously. Results also apply for IVF-ICSI.

The efficacy found in this analysis applies for a time interval of two to three months between tubal occlusion or salpingectomy and IVF. Results apply for unilateral of tubal occlusion or salpingectomy in case of a unilateral hydrosalpinx and bilateral employment in case of bilateral surgery. There is no evidence for bilateral tubal salpingectomy or occlusion regardless of the distribution of tubal pathology, because this has only been assessed for salpingectomy by one study (Darwish 2005,2006, 2007).

An important question in evaluating the efficacy of interventions is 'who can achieve the reported results from these interventions?' A weakness of included studies is that information on the skills of the performer is not provided. Despite the fact that some methods for tubal occlusion might require less surgical skill, we recommend, in agreement with the general opinion, that tubal surgery should only be performed by gynaecologists who have appropriate training in microsurgery or laparoscopy and who operate on a steady volume of patients to maintain a high level of skill (Dechaud 2004).

This review reflects the opinion that definitions of fertility should be sharpened (Clarke 2008). Therefore we focused primarily on live birth, and secondarily on ongoing pregnancy and viable, clinical or biochemical pregnancy. Sadly however, studies either did not report on live birth, or combined ongoing pregnancy results with live birth rates. In the last case data was classified under ongoing pregnancy. As studies do not always report pregnancy strictly the category: pregnancy according to any definition was used. This was done to prevent loss of data; this was specified and outcomes should be regarded with care.
As there are numerous suggested outcomes for ovarian response; and we were only able to assess differences in the mean number of oocytes retrieved, as reported by the trials themselves, the conclusion should be interpreted with caution. More evaluation of the difference in ovarian response prior to and after salpingectomy should be performed and compared with other interventions. Other measures of ovarian reserve could be considered as other outcome measures.

Study size and power has been proven to be an important issue in the previous version of this review where reviewed outcomes were not significant prior to meta-analysis but significant after metaanalysis. Generally, all studies but one (Kontoravdis 2006) were underpowered. In the newly added comparison of tubal occlusion, study size was sufficient to show important differences for the the outcome clinical pregnancy after meta-analysis; the fact that the odds of ongoing pregnancy were not significantly improved is probably due to the fact that only one study reported this outcome. This also applies for the results of aspiration, where outcomes also depend on only one trial. Adverse effects naturally have lower event rates and thus need a larger sample size to reach significance, thus ectopic pregnancy and miscarriage were underpowered adverse events. Studies report the difficulty to adhere to power calculations because the expected number of recruits was too large for a single department (Dechaud 1998). Furthermore drawbacks of a multicentre trial are differences in surgical skill and possibly sample selection. This is a difficult matter to be addressed in attaining sample size.

Data of four studies reporting on laparoscopic salpingectomy, including two that also reported on laparoscopic tubal occlusion, were pooled for meta-analysis. Was it appropriate to pool the data from these trials? In general pooling was appropriate because trials were clinically similar regarding the extent of tubal pathology and the procedure employed in both the intervention and the control group. There were however some considerations regarding pooling; it should be explained as to why pooling was still justified in these cases.

A clinical consideration in the salpingectomy group was the trial of Dechaud 1998. This trial differed from other studies by the fact that salpingectomy was employed bilaterally regardless of the distribution of hydrosalpinges. Other studies in this pool employed uni or bi-lateral salpingectomy depending on whether the hydrosalpinx was uni or bi-lateral (Kontoravdis 2006; Strandell 1999) or bilateral by definition as all patients included had hydrosalpinges bilaterally (Moshin 2006). As in essence, all four trials compared a group who underwent laparoscopic surgery to remove on a fallopian tubes to thus prevent hydrosalpinx fluid spill into the uterine cavity versus a group who did not have this surgical intervention, data were pooled. There was no statistical heterogeneity for outcomes where the trial of Dechaud 1998 was involved, supporting the decision to pool the data.

Regarding analysis of data on the efficacy of tubal occlusion a consideration was that the methods of occlusion differed: Kontoravdis 2006 used diathermy to achieve occlusion; Moshin 2006 used clamping. This difference was dealt with by classifying trials in different subgroups. Pooling of data of Kontoravdis 2006 and Moshin 2006 however seemed acceptable because the pathophysiologic mechanism of occlusion, regardless of the method, seems similar. Pooling was supported because there was 
no statistical heterogeneity for outcomes in this comparison group supporting the decision to pool the data.

Pooling of Kontoravdis 2006; Moshin 2006and Strandell 1999 shows a moderate statistical heterogeneity. As clinical considerations were similar, heterogeneity was not present consistently in all outcomes and as this statistical heterogeneity was moderate: heterogeneity was accepted and studies were pooled.

Results of miscarriage were not pooled as definitions of this outcome was not clear.

\section{Quality of the evidence}

Risk of bias assessment revealed a predisposition to detection bias, financial bias and possibly to publication bias.A detection bias may be present throughout all studies, as blinding was either not reported to be performed or not performed. The importance of patient blinding is relative, besides the limited feasibility due to ethical and practical considerations, as the extent to which patients can influence there IVF outcome is minimal.Whether physicians and outcome assessors were blinded may be more important, as knowledge of allocation could influence the physicians in the IVF procedure and further follow-up. Blinding of participants and outcome assessors would have been possible in all trials.

Detection bias may lead to overinflation of treatment effects.The lack of report on the source of funding was a consideration in one trial (Dechaud 1998). This is often a concern due to reporting rather than bias because of financial motives of an internal or external party involved in the trial.

The most important consideration was power; as addressed before. The main reasons for not adhering to power calculations was the difficulty to recruit the calculated number of patients. This may be justified for a single centre trial and to a lesser extent for multicenter trials as hydrosalpinges do not present as often as other indications for IVF. Power issues underline the importance of meta-analysis, however they may also generate a predisposition to other bias. Particularly in unblinded studies, where unblinded participating clinicians may be aware of the results (with or without preliminary analyses) and therefore be biased to cease the trial earlier. Hammadieh 2008 was the only trial to state how it was decided to cease recruitment; therefore in the remaining cases it is difficult to assess whether and when other factors might have been considered in the decision to cease recruitment, leaving space for potential bias of results.

Summarising, theoretically detection bias, financial bias, a publication bias and unknown bias leading to early cessation may be present. It is however difficult to estimate the effect on the outcomes.

\section{Potential biases in the review process}

The strength of this review lies in the application of Cochrane methodology. The review was conducted according to the predefined plan. An extensive search was applied therefore we believe that the likelihood that all relevant studies were identified is quite high. In general data was obtained quite complete, a limitations was the fact that one trial was an abstract and that some uncertainties of lesser importance remain unclear because of time restrictions in the review process.

\section{Agreements and disagreements with other studies or reviews}

Prior to the first version of this review it was generally accepted that women with hydrosalpinges had a reduced likelihood of success from IVF. Although the efficacy of surgical treatment of hydrosalpinges had not been demonstrated in randomised trials, many clinicians were convinced of the beneficial effect by conducted non-randomised studies (Poe-Ziegler 1995; Vandromme 1995; Andersen 1996; Puttemans 1996, Shelton 1996). Surgical interventions were performed by a majority of the clinicians (Johnson 2002). The first version of this review showed a statistically significant benefit of laparoscopic salpingectomy for hydrosalpinges prior to IVF, after meta-analysis of randomised, individually non significant studies for the first time The current update is in line with this finding and further supported the beneficial effect of salpingectomy.

Previous to the RCT's included in this Cochrane review the beneficial effect of tubal occlusion by electrocautery was only suggested by three retrospective studies Stadtmauer 2000; Murray 1998; Surrey 2001). Results seemed beneficial, and for some clinicians convincing enough (Ducarme 2006; Johnson 2002; Hammadieh 2004). Prior to the current version of the review no randomized controlled trials were included to report the efficacy of tubal occlusion. After inclusion of Kontoravdis 2006 and Moshin 2006 this current review now provides the evidence, based on randomized controlled trials, that laparoscopic tubal occlusion improves clinical pregnancy rates achieved with IVF. The effects on IVF outcome of other methods and approaches for tubal occlusion than laparoscopic occlusion by electrocautery, in women with hydrosalpinges have not been addressed by randomised controlled trials. As tubal occlusion is now shown to be beneficial by this review; further evaluation of other methods for tubal occlusion would be helpful.

Ultrasound guided aspiration was the first reported therapeutic option (Aboulghar 1990; Russel 1991; Sowter 1997) since the acknowledgement of the deleterious effect of hydrosalpinges. However, up to the previous Cochrane review efficacy of aspiration had not been shown in a randomised controlled trial. Previous trials consisted of two retrospective comparative studies (Sowter 1997; Van Voorhis 1998), two non-randomised studies (Shahara 1996; Aboulghar 1990) and two case reports (Bloechle 1997; Russel 1991). Results of these studies were conflicting and weak.

This meta-analysis failed to show a significant effect on the odds of miscarriage. Other literature on miscarriage is conflicting (Andersen 1994; Zeyneloglu 1998; Shahara 1996). The same literature does not show a significant effect on the odds of ectopic pregnancy, event rates were, as in the included RCT's low. Whilst it is rational to expect a reduction in the likelihood of ectopic pregnancy following salpingectomy for a hydrosalpinx, this meta-analysis was underpowered to demonstrate a significant difference in the odds of ectopic pregnancy.

Our findings on ovarian function are limited and therefore cannot provide evidence as to whether salpingectomy could adversely affect ovarian ovarian reserve. Although Strandell 2001 was reassuring as to the integrity of ovarian function after salpingectomy and a recent retrospective study concluded that salpingectomy may not compromise ovarian function without significant differences in pregnancy (Gelbaya 2006). As there is not 
enough evidence to resolve this matter; dissection must therefore be performed very close to the fallopian tube to avoid disrupting the ovarian blood supply. Furthermore we cannot support nor reject the hypothesis of Lass 1998: who suggested that proximal clamping and distal fenestration of the fallopian tube may avoid the problem of disruption of blood supply, an approach used in the Strandell 1999 trial if extensive adhesions were present.

\section{AUTHORS' CONCLUSIONS}

\section{Implications for practice}

Surgical treatment should be considered for all women with hydrosalpinges who are due to undergo IVF. Laparoscopic salpingectomy prior to IVF treatment increases the odds of ongoing pregnancy and clinical pregnancy versus no treatment in the short term. Furthermore, this review now shows that laparoscopic tubal occlusion presents an alternative to laparoscopic salpingectomy, as the odds of clinical pregnancy are improved and comparison of tubal occlusion with salpingectomy has failed to show a difference in effectiveness.

\section{Implications for research}

Firstly, randomised controlled trials need to further assess the efficacy of individual treatments:

a) ultrasound guided aspiration

b) salpingostomy

c) tubal occlusion (approaches and methods of occlusion (laparoscopically by electrocautery or clips or hysteroscopically by placement of micro inserts or electrocautery) need to be compared.

Research should have a further scope and compare efficacy amongst different surgical treatments: preferably less invasive techniques (tubal occlusion, aspiration, salpingostomy) compared to invasive surgery (salpingectomy). Interventions should be compared in terms of:
a) live birth
b) ongoing pregnancy
b) clinical pregnancy
c) adverse effects.

Secondly, the place of surgical treatment of hydrosalpinges in clinical practice needs to be defined:

(1) The spectrum of tubal disease that surgical treatment is beneficial for:

(a) the further clarification of the benefit to women whose hydrosalpinges are not visible on ultrasound. (b) tubal surgery in the context of tubal disease without hydrosalpinx formation.

(2) Adverse effects:

(a) Influence on ovarian function

(3) Timing of treatment:

(a) Time frame to IVF

(b) With or without IVF - does surgical treatment positively affect spontaneous pregnancy rates in women with at least one patent fallopian tube, compared to pregnancy rates after IVF treatment?

(c) Efficacy of surgical intervention before IVF or after previously failed IVF.

\section{(4) Comparison to restorative surgery:}

If restorative tubal surgery and specialised diagnostic facilities such as salpingoscopy are available, their appropriate use would be best determined by further RCTs in well-defined populations. Examples of such trials which would support the suggestions of authors mentioned in the discussion section would be:

(1) restorative tubal surgery versus surgical intervention and IVF in salpingo scopically selected women with hydrosalpinges;

(2) laparoscopic salpingostomy versus laparoscopic salpingectomy prior to IVF for women with thin-walled hydrosalpinges which are free from ampullary adhesions.

In general, outcomes of subfertility should be strictly be expressed primarily in live birth rates and secondarily ongoing pregnancy rates, or viable- or clinical pregnancy rates (as defined in the methods section of this review). All trials should have adequate power, with pregnancy outcomes ideally expressed as a continuous time survival analysis. As an example, a sample size of about 600 would give $80 \%$ power at the $95 \%$ confidence interval to detect differences in pregnancy outcomes of $30 \%$ versus $20 \%$ in the two groups.

\section{ACKNOWLEDGEMENTS}

The authors acknowledge the helpful comments of those who have refereed this review and the authors of included trials who supplied additional information or data in the previous and current review, particularly Annika Strandell, Evangelos Makrakis, Herve Déchaud, Nahed Hammadieh and Vaeceslav Moshin. We wish to thank Jane Clarke, MDSG Managing Editor, and Marian Showell, MDSG Trials Search Coordinator. Stephan Bontekoe assisted with copy editing in RevMan 5. 


\section{R E F E R E N C E S}

\section{References to studies included in this review}

Dechaud 1998 \{published data only\}

* Dechaud H, Daures JP, Amal F, Humeau C, Hedon B. Does previous salpingectomy improve implantation and pregnancy rates in patients with severe tubal factor infertility who are undergoing in vitro fertilization? A pilot prospective randomized study. Fertility \& Sterility 1998;69:1020-5.

Dechaud H, Daures JP, Arnal F, Humeau C, Hedon B. Salpingectomy before undergoing IVF can increase implantation rates in severe tubal infertility patients: a prospective randomised study. Human Reproduction Abstracts of 13th Annual Meeting of the ESHRE. 1997; Vol. 12:23-4.

Hammadieh 2008 \{published data only\}

Hammadieh N, Afnan M, Sharif K, Evans J, Amso N. The effect of hydrosalpinx on IVF outcome: a prospective randomised controlled trial of vaginal ultrasound-guided hydrosalpinx aspiration during egg collection. Fertility \& Sterility 2003; Vol. 80 (Suppl. 3):S131-132.

* Hammadieh N, Coomarasamy A, Bolarinde O, Papaioannou S, Afnan M, Sharif K. Ultrasound-guided hydrosalpinx aspiration during oocyte collection improves pregnancy outcome in IVF: a randomized controlled trial. Human Reproduction 2008;23:1113-7.

\section{Kontoravdis 2006 \{published data only\}}

Kontoravdis A, Makrakis E, Pantos K, Botsis D, Deligeoroglu E, Creatsas G. Proximal tubal occlusion and salpingectomy result in similar improvement in in vitro fertilization outcome in patients with hydrosalpinx. Fertility and Sterility December 2006;Vol 86:1642-8.

\section{Moshin 2006 \{published data only\}}

Moshin V, Hotineanu A. Reproductive outcome of the proximal tubal occlusion prior to IVF in patients with hydrosalpinx. Human reproduction. June 2006; Vol. 21:i193-i194.

\section{Strandell 1999 \{published data only\}}

* Strandell A, Lindhard A, Waldenstrom U, Thorburn J, Janson PO, Hamberger L. Hydrosalpinx and IVF outcome: a prospective randomized multicentre trial in Scandinavia on salpingectomy prior to IVF. Human Reproduction 1999;14(11):2762-9.

Strandell A, Lindhard A, Waldenstrom U, Thorburn J, Janson PO, Hamberger L. Hydrosalpinx and IVF outcome: cumulative results after salpingectomy in a randomised controlled trial. Human Reproduction 2001;16:2403-10.

\section{References to studies excluded from this review}

\section{Gelbaya 2006 \{published data only\}}

Gelbaya TA, Nardo LG, Fitzgerald CT, Horne G, Brison DR, Lieberman BA. Ovarian response to gonadotrophins after laparoscopic salpingectomy or the division of fallopian tubes for hydrosalpinges. Fertility and Sterility 2006;85(5):1464-8.
Goldstein 1998 \{published data only\}

Goldstein DB, Sasaran LH, Stadtmauer L, Popa R. Selective salpingostomy-salpingectomy (SSS) and medical treatment prior to IVF in patients with hydrosalpinx. Fertility \& Sterility 1998;70(1):S320.

\section{Hotineanu 2007 \{published data only\}}

Hotineanu 2007 Hotineanu AL, Moshin VN, Hotineanu AV, Croitor ME. The effect of proximal tubal "clamping" prior to the IVF in patients with distal tubal occlusion. Human Reproduction July 2007;22 (Supplement 1; Abstracts of the 23rd Annual Meeting of the European Society of Human Reproduction and Embryology):i126.

\section{Mardesic 1999 \{published data only\}}

Mardesic T, Muller P, Huttelová R, Zvárová J, Hulvert J, Voboril J, et al. Effect of salpingectomy on the results of IVF in women with tubal sterility--prospective study. Ceska Gynekol. 2001;66(1):259-64.

Mardesic T, Muller P, Voboril J, Hulvert J, Huttelova R, Becvarova $\mathrm{V}$, et al. The influence of salpingectomy of hydrosalpinges visible on ultrasound on IVF results. A pilot prospective randomized study. Abstracts of 11th World Congress on In Vitro Fertilization and Human Reproductive Genetics. Sydney, Australia, 9-14 May, 1999:156.

\section{Zolghadri 2006 \{published data only\}}

Zolghadri J, Momtahan M, Alborzi S, Mohammadinejad A, Khosravi D. Pregnancy outcome in patients with early recurrent abortion following laparoscopic tubal corneal interruption of a fallopian tube with hydrosalpinx.. Fertility and Sterility July 2006;86(6):149-51.

\section{References to studies awaiting assessment}

Hammadieh2008 \{published data only\}

Hammadieh N, Coomarasamy A, Ola B, Papaioannou S, Afnan M, Sharif K. Ultrasound-guided hydrosalpinx aspiration during oocyte collection improves pregnancy outcome in IVF: a randomized controlled trial. Human reproduction 2008;23(5):1113-7.

\section{Mol 2008 \{published data only\}}

Mol F, Strandell A, Jurkovic D, Yalcinkaya T, Verhoeve HR, et al. The ESEP study: salpingostomy versus salpingectomy for tubal ectopic pregnancy; the impact on future fertility: a randomised controlled trial. BMC women's health 2008;8:11.

\section{References to ongoing studies}

Darwish 2005,2006, 2007 \{published data only\}

Darwish A, El Saman E. Hysteroscopic vs laparoscopic tubal occlusion of hydrosalpinges prior to IVF/ICSI. Human Reproduction 2006;21:i134.

Darwish A, ElSaman E. Hysteroscopic versus laparoscopic tubal occlusion of hydrosalpinges prior to IVF/ICSI. 
Middle-East Fertility Society Journal 2005;10(Supplement 12th Annual Meeting of the Middle East Fertility Society 23-26November):i126

Darwish AM, El Saman AM. Is there a role for hysteroscopic tubal occlusion of functionless hydrosalpinges prior to IVF/ IVCSI in modern practice?. Acta Obstetrica et Gynaecologica 2007;86:1484-9.

\section{Additional references}

\section{Aboulghar 1990}

Aboulghar MA, Mansour RT, Serour GI, Sattar MA, Awad MM, Amin Y. Transvaginal ultrasonic needle guided aspiration of pelvic inflammatory masses before ovulation induction for in vitro fertilization. Fertility \& Sterility 1990;53:311.

\section{Akman 1996}

Akman MA, Garcia JE, Damewood MD, Watts LD, Katz E. Hydrosalpinx affects the implantation of previously cryopreserved embryos. Human Reproduction 1996;11:1013-4.

\section{Andersen 1994}

Andersen A, Yue Z, Meng F, Petersen K. Low implantation rate after in-vitro fertilisation in patients with hydrosalpinges diagnosed by ultrasonography. Human Reproduction 1994;9:1935-8.

\section{Andersen 1996}

Andersen AN, Lindhard A, Loft A, Ziebe S, Andersen CY. The infertile patient with hydrosalpinges: IVF with or without salpingectomy?. Human Reproduction 1996;11:2081-4.

\section{Bloechle 1997}

Bloechle M, Schreiner Th, Lisse K. Recurrence of hydrosalpinges after transvaginal aspiration of tubal fluid in an IVF cycle with development of a serometra. Human Reproduction 1997;12:703-5.

\section{Camus 1999}

Camus E, Poncelet C, Goffinet F, Wainer B, Merlet F, Nisand I, et al. Pregnancy rates after in-vitro fertilization in cases of tubal infertility with and without hydrosalpinx: a meta-analysis of published comparative studies. Human Reproduction May 1999;14(5):1243-9.

\section{Clarke 2008}

JF Clarke, M van Rummste, C Farquhar, N Johnson, BW Mol, $\mathrm{P}$ Herbison. Measuring outcomes in fertility trials - can we rely on clinical pregnancy rates?. Submitted for publication.

\section{Csemiczky 1996}

Csemiczky G, Landgren BM, Fried G, Wramsby H. High tubal damage grade is associated with low pregnancy rate in women undergoing in vitro fertilisation treatment. Human Reproduction 1996;11:2438-40.

\section{Dar 2000}

Dar P, Sachs GS, Strassburger D, et al. Ovarian function before and after salpingectomy in artificial reproductive technology patients. Human Reproduction 2000;15:142-4.

\section{Dechaud 2004}

Dechaud H, Reyftmann L, Faidherbe, Hamamah S, Hedon B. Evidence based reproductive surgery: tubal infertility. International congress series 2004;1266:96-106.

\section{Dias 2006}

Dias S, McNamee R, Vail A. Evidence of improving quality of reporting of randomized controlled trial in subfertility. Human Reproduction 2006;21:2617-27.

\section{Dickens 1995}

Dickens CJ, Maguiness SD, Comer MT, Palmer S, Rutherford AJ, Leese HJ. Human tubal fluid: formation and composition during vascular perfusion of the Fallopian tube. Human Reproduction 1995;10:505-8.

\section{Ducarme 2006}

Ducarme G, Uzan M, Hugues JN, Cedrin-Durnerin I, Ponselet C. Management of hydrosalpinx before or during in vitro fertilization -embryo transfer: a national postal survey in France. Fertility and Sterility 2006;86(4):1013-16.

\section{Englert 1987}

Englert Y, Vekemans M, Lejeune B, Van Rysselberge M, Puissant F, Degueldre M, et al. Higher pregnancy rates after in vitro fertilization and embryo transfer in cases with sperm defects. Fertility \& Sterility 1987;48:254-7.

\section{Evers 2002}

Evers JL. Female subfertility. The Lancet 2002;360(9327):151-9.

\section{Eytan 2001}

Eytan O, Azem F, Gull I, Wolman I, Elad D, Jaffa AJ. The mechanism of hydrosalpinx in embryo implantation. Human Reproduction 2001;12:2662-7.

\section{Fleming 1996}

Fleming C, Hull MGR. Impaired implantation after in vitro fertilisation treatment associated with hydrosalpinx. British Journal of Obstetrics \& Gynaecology 1996;103:268-72.

\section{Freeman 1996}

Freeman MR, Whitworth CM, Hill GA. Hydrosalpinx reduces in vitro fertilisation / embryo transfer rates and in vitro blastocyst development. 52nd Annual Meeting of the American Fertility Society. 1996:S211.

\section{Hammadieh 2004}

Hammadieh N, Afnan M, Evans J, Sharif K, Amso N, Olufowobi O. A postal survey of hydrosalpinx management prior to IVF in the United Kingdom. Human Reproduction 2004;19(4):1009-12.

\section{Herman 1991}

Herman A, Ron-el R, Golan A, Soffer Y, Bukovsky Y, Caspi E. The dilemma of the optimal surgical procedure occurring in ectopic pregnancies in in-vitro fertilisation. Human Reproduction 1991;6:1167-9.

\section{Higgins 2008}

Higgins JPT, Green S (editors). Cochrane Handbook for Systematic Reviews of Interventions Version. 5.0.0. The 
Cochrane Collaboration, February 2008. Available from www.cochrane-handbook.org.

\section{Hsu 2005}

Hsu CC, Yang TT, Hsu CT. Ovarian pregnancy resulting from cornual fistulae in a woman who had undergone bilateral salpingectomy. Fertility \& Sterility 2005;83(1):205-7.

\section{Inovay 1999}

Inovay J, Marton T, Urbancsek J, et al. Spontaneous bilateral cornual dehiscence early in the second trimester after bilateral laparoscopic salpingectomy and in vitro fertilization. Human Reproduction 1999;14:2471-3.

\section{Johnson 2002}

Johnson NP, Merrilees M, Sadler L. IVF and tubal pathology - not all bad news. Australian and New Zealand Journal of Obstetrics \& Gynaecology 2002;42:285-8.

\section{Johnson2002}

Johnson NP, Norris J. An Australasian survey of the management of hydrosalpinges in women due to undergo in vitro fertilisation. The Australian and New Zealand Journal of Obstetrics and Gynaecology 2002;42(3):271-6.

\section{Katz 1996}

Katz E, Akman MA, Damewood MD, Garcia JE. Deleterious effect of the presence of hydrosalpinx on implantation and pregnancy rates with in vitro fertilisation. Fertility \& Sterility 1996;66:122-5.

\section{Kerin 2005}

Kerin J, Swann N, Scroggs S, Rosenfield R. Hysteroscopic placement of Essure micro-inserts to obstruct hydrosalpinges followed by IVF and successful pregnancy outcomes. Australian and New Zealand Journal of Obstetrics and Gynaecology 2005;45(s(1)):A11.

\section{La Combe 2003}

LaCombe. Adnexal torsion in a patient with hydrosalpinx who underwent tubal occlusion before in vitro fertilization. Fertility \& Sterility 2003;79(2):437-8.

\section{Lass 1998}

Lass A, Ellenbogen A, Croucher C, et al. Effect of salpingectomy on ovarian response to superovulation in an in vitro fertilization-embryo transfer program. Fertility \& Sterility 1998;70:1035-8.

\section{Mansour 1991}

Mansour RT, Aboulghar MA, Serrour GI, Riad R. Fluid accumulation of the uterine cavity before embryo transfer: a possible hindrance for implantation. Journal of In vitro fertilisation and Embryo Transfer 1991;8:157-9.

\section{Meyer 1997}

Meyer WR, Castelbaum AJ, Somkuti S, Sagoskin AW, Doyle M, Harris JE, et al. Hydrosalpinges adversely affect markers of endometrial receptivity. Human Reproduction 1997;12:1393-8.

\section{Mukherjee 1996}

Mukhurjee T, Copperman AB, McCaffrey C. Hydrosalpinx fluid has embryotoxic effects on murine embryogenesis: a case for prophylactic salpingectomy. Fertility \& Sterility 1996;66:851-3.

\section{Murray 1998}

Murray DL, Sagoskin AW, Widra EA, Levy MJ. The adverse effect of hydrosalpinges on in vitro fertilisation pregnancy rates and the benefit of surgical correction. Fertility \& Sterility 1998;69(1):619-26.

\section{Poe-Ziegler 1995}

Poe-Ziegler R, Shelton KE, Toner JP. Salpingectomy(ies) improves the pregnancy rate after IVF in patients with unilateral or bilateral hydrosalpinx. Journal of Assisted Reproduction \& Genetics 1995;12:S65.

\section{Puttemans 1996}

Puttemans PJ, Brosens IA. Salpingectomy improves in vitro fertilisation outcome in patients with a hydrosalpinx: blind victimisation of the fallopian tube?. Human Reproduction 1996;11:2079-81.

\section{Rosenfield 2005}

Rosenfield RB, Stones RE, Coates A, Matteri RK, Hesla JS. Proximal occlusion of hydrosalpinx by hysteroscopic placement of microinsert before in vitro fertilization embryo transfer. Fertility \& Sterility 2005;83(5):1547.

\section{Russel 1991}

Russel JB, Rodriguez Z, Komins JI. The use of transvaginal ultrasound to aspirate bilateral hydrosalpinges prior to in vitro fertilisation: a case report. Journal of In vitro fertilisation and Embryo Transfer 1991;8(4):213-5.

\section{Savic 1999}

Savic B, Milacic D, Peako N. Hydrosalpingeal fluid aspiration during oocyte retrieval has beneficial effect on outcome of invitro fertilization-embryo transfer. Abstracts of the 15th Annual Meeting of the European Society of Human Reproduction and Embryology. 1999:310.

\section{Shahara 1996}

Shahara FI, Scott RT Jr, Marut EL, Queenan JT Jr. In-vitro fertilisation outcome in women with hydrosalpinx. Human Reproduction 1996;11:526-30.

\section{Sharara 1999}

Sharara FI. The role of hydrosalpinx in IVF: simply mechanical? Human Reproduction 1999;14:577-8.

\section{Shariff 1994}

Shariff K, Kaufmann S, Sharma V. Heterotopic pregnancy obtained after In-vitro fertilization and embryo transfer following bilateral total salpingectomy: case report. Human Reproduction 1994;9:1966-7.

\section{Shelton 1996}

Shelton KE, Butier L, Toner JP. Salpingectomy improves the pregnancy rate in in vitro fertilisation patients with hydrosalpinx. Human Reproduction 1996;11:523-5. 


\section{Sowter 1997}

Sowter MC, Akande VA, Williams JA, Hull MG. Is the outcome of in-vitro fertilisation and embryo transfer treatment improved by spontaneous or surgical drainage of a hydrosalpinx?. Human Reproduction 1997;12:2147-50.

\section{Stadtmauer 2000}

Stadtmauer LA, Riehl RM, Toma SK, Talbert LM. Cauterization of hydrosalpinges before in vitro fertilization is an effective surgical treatment associated with improved pregnancy rates. American Journal of Obstetrics \& Gynecology 2000;183:367-71.

\section{Steptoe 1978}

Steptoe PC, Edwards RG. Birth after the reimplantation of a human embryo. Lancet 1978;2:366.

\section{Strandell 1994}

Strandell A, Waldenstrom U, Nilsson L, Hamberger L. Hydrosalpinx reduces in-vitro fertilisation / embryo transfer pregnancy rates. Human Reproduction 1994;9:861-3.

\section{Strandell 2001}

Strandell A, Lindhard A, Waldenstrom U, et al. Salpingectomy prior to IVF does not impair the ovarian response. Human Reproduction 2001;16:1135-9.

\section{Strandell 2002}

Strandell A, Lindhard A. Why does hydrosalpinx reduce fertility; the importance of hydro salpingeal fluid. Human Reproduction 2002;17(5):1141-5.

\section{Surrey 2001}

Surrey AS, Schoolcraft WB. Laparoscopic management of hydrosalpinges before in vitro fertilization embryo transfer: salpingectomy versus proximal tubal occlusion. Fertility \& Sterility March 2001;75(3):612-7.

\section{CHARACTERISTICS OF STUDIES}

Characteristics of included studies [ordered by study ID]

\section{Tay 1997}

Tay JI, Rutherford AJ, Killick SR, Maguiness SD, Partridge RJ, Leese HJ. Human tubal fluid: production, nutrient composition and response to adrenergic agents. Human Reproduction 1998;70:492-9.

\section{Taylor 2001}

Taylor RC, Berkowitz J, McComb PF. Role of laparoscopic salpingostomy in the treatment of hydrosalpinx. Fertility and Sterilty 2001;75:594-600.

\section{Van Voorhis 1998}

Van Voorhis BJ, Sparks AET, Syrop CH, Stovall DW. Ultrasoundguided aspiration of hydrosalpinges is associated with improved pregnancy and implantation rates after in-vitro fertilization cycles. Human Reproduction 1998;13:736-9.

\section{Vandromme 1995}

Vandromme J, Chasse E, Lejeune B, Van Rysselberge M, Delvigne A, Leroy F. Hydrosalpinges in in vitro fertilisation: an unfavourable prognostic feature. Human Reproduction 1995;10:576-9.

\section{Vasquez 1995}

Vasquez G, Boeckx K, Brosens IA. Prospective study of tubal mucosal lesions and fertility in hydrosalpinges. Human Reproduction 1995;10:1075-8.

\section{Zeyneloglu 1998}

Zeyneloglu HB, Arici A, Olive DL. Adverse effects of hydrosalpinx on pregnancy rates after in vitro fertilization - embryo transfer. Fertility \& Sterility 1998;70:492-9.

* Indicates the major publication for the study

\section{Dechaud 1998}

Methods Randomised controlled trial.

Single-centre, Department of Obstetrics and Gynaecology at a university hospital (Montpelier, France). Patient sampling: not explained.

Inclusion criteria: women with severe tubal infertility, not suitable for tubal repair. Exclusion criteria: age >40 years; additional causes of infertility; tubal pathology suitable for repair by tubal catheterization, laparoscopic surgery, or microsurgical techniques; tubal pathology so severe as to require bilateral salpingectomy; lack of patient consent for salpingectomy or randomisation.

60 women recruited, 60 randomised (30 to laparoscopic bilateral salpingectomy and adhesiolysis; 30 to laparoscopic adhesiolysis).

Number excluded not known.

No loss to follow up.

60 women analysed.

Follow-up duration 1-5 years. Follow-up scheme: not specified.

Participants Age $</=40$ years (range 27-36).


Type (primary or secondary) of infertility: not stated.

Duration of infertility, mean months (SD): laparoscopic salpingectomy group 55.2 (33.3); laparoscopic adhesiolysis group 48.0 (25.4).

Investigative work-up: baseline FSH, endocrine laboratory screening, semen analysis, post coital test, laparoscopy and confirmatory test of ovulation.

Other contributory causes: none

Previous treatment: no previous IVF.

Diagnosis of tubal pathology: by laparoscopy and(or) HSG; diagnostic criteria are specified.

Extent of tubal pathology: salpingitis isthmica nodosa or hydrosalpinx with a poor prognosis (dis-

turbed with mucosal folds or irregular walls on HSG; proximal nodes or a thick walled hydrosalpinx).

Distribution: unclear.

Characteristics of IVF treatment:

IVF protocol: a GnRH agonist protocol with hMG and hCG.

Number of IVF ovarian stimulation cycles per woman: not stated.

Mean oocytes retrieved per cycle (SD): 10.1 (5.0) in the salpingectomy group; 10.5 (6.0) in the laparoscopic adhesiolysis group. No ICSI. Fertilization rate: not stated. Mean number of embryos transferred (SD): 3.3 (1.2) in the salpingectomy group; 3.4 (1.2) in the laparoscopic adhesiolysis; up to 5 embryos were replaced in some cases.

Criteria for surgical treatment prior to IVF: a communicating non-draining hydrosalpinx with salpingitis isthmica nodosa.

Mean interval from surgical intervention to IVF in months (SD): treatment group 10.1 (7.5); control group 9.5 (7.2).

Cointerventions: none stated.

Data on performer: not stated.

Outcomes

PRIMARY OUTCOMES

Ongoing pregnancy rate - not defined; reported per transfer.

Pregnancy rate - not defined; reported per transfer, per oocyte retrieval, per IVF cycle.

Cumulative pregnancy rates were presented also and obtained with the use of cumulative proportion test (the cumulative probability of becoming pregnant after each IVF attempt according to the number of patients, the number of pregnancies for each IVF attempt and the number of patients who discontinued IVF after each IVF attempt). The investigators hypothesized that the likelihood of becoming pregnant would have been equal for the patients who became pregnant after IVF and for those who discontinued IVF treatment.

\section{SECONDARY OUTCOMES}

Implantation rate - not defined.

Proportion of IVF cycles resulting in embryo transfer.

Ectopic pregnancy and miscarriage rate - not defined.

Notes

Subsequent publication of the cumulative results from this trial (Strandell 2001) were excluded from meta-analysis (not intention to treat and too many protocol breached when intention to treat data was provided).

\section{Risk of bias}

\begin{tabular}{lll}
\hline Bias & Authors' judgement & Support for judgement \\
\hline Allocation concealment? & Low risk & With sealed opaque envelopes. \\
\hline $\begin{array}{l}\text { Blinding? } \\
\begin{array}{l}\text { All outcomes. Partici- } \\
\text { pants, outcome assessors, } \\
\text { clinicians, statisticians }\end{array}\end{array}$ & High risk & No blinding. \\
\hline
\end{tabular}




\section{Dechaud 1998 (Continued)}

Incomplete outcome data addressed?

Intention to treat for pri-

mary outcome?
Low risk Intention to treat analysis is unclear; but number randomised is same as number analysed.

\begin{tabular}{|c|c|c|}
\hline $\begin{array}{l}\text { Free of selective report- } \\
\text { ing? }\end{array}$ & Low risk & No suggestion of selective reporting. \\
\hline Detection adequate? & Low risk & Sufficient length of follow-up: 1-5 years. \\
\hline Source of funding stated? & Unclear risk & Presence or absence of funding is not reported. \\
\hline $\begin{array}{l}\text { Powercalculation per- } \\
\text { formed? }\end{array}$ & High risk & $\begin{array}{l}\text { A power calculation was performed but not adhered to as the number calcu- } \\
\text { lated ( } 322 \text { patients in each group) would not be achieved in the setting of the } \\
\text { trial. When the trial was ceased and the exact reasons for cessation are un- } \\
\text { known. }\end{array}$ \\
\hline $\begin{array}{l}\text { Loss to Follow-up ex- } \\
\text { plained? }\end{array}$ & Low risk & No withdrawals or losses to follow-up. \\
\hline
\end{tabular}

\section{Hammadieh 2008}

Methods Randomised controlled trial.

Single centre, non academic, trial in the Assisted Conception Unit of Birmingham Women's Hospital, UK.

Patient sampling unclear.

Inclusion criteria: a uni- or bilateral hydrosalpinx diagnosed by ultrasound during ovarian stimulation in the IVF cycle or previous to the IVF cycle, or women with severe tubal pathology on the waiting list for salpingectomy for hydrosalpinges.

Exclusion criteria: other major fertility factors.

66 women recruited and randomised ( 32 to aspiration; 34 to no intervention). 1,154 exclusions prior to randomisation. No women withdrew or lost to follow up. 66 women were analysed.

Follow-up duration up to 1 IVF cycle. Type of follow-up: biochemical pregnancy 14 days post ET; if positive ultrasound examination is performed 6 weeks post transfer. After diagnosis of viable pregnancy patients are referred to their GP.

Participants

Age (years (SD)): aspiration group: $33.4 \pm 4.5$ / no intervention group 33.9 \pm 4.7 .

Type (primary/secondary) of subfertility: primary infertility 32/66 (aspiration group 41\%, control group $56 \%)$.

Duration of infertility: not stated.

Previous investigative work-up: not stated other than baseline FSH levels.

Contributary causes to infertility: exclusion criterion.

Previous treatments: no previous IVF treatment; not otherwise specified.

Diagnosis of tubal pathology: by ultrasound and by DLS or HSG in 7 patients.

Characteristics of tubal pathology: hydrosalpinges. 14 bilateral ( 8 in the aspiration group; 6 in the non intervention group).

Characteristics of IVF treatment:

Ovarian stimulation protocol: long antagonist protocol.

Single IVF ovarian stimulation cycle per woman.

Mean oocytes retrieved at IVF (SD) - aspiration group 14.1 (7.1); non intervention group 12.4 (7.1).Proportion undergoing ICSI, 5\% aspiration group; $7 \%$ non-intervention group. Mean no. of fertilized 
Hammadieh 2008 (Continued)

oocytes in the aspiration group 9.2 (5.2); 7.0 (4.6) in the non-aspiration group. Mean embryos transferred per cycles (SD) - 2.3 (0.58) in the aspiration group; 2.1 (0.64) in the control group.

Interventions

Aspiration of hydrosalpinx or hydrosalpinges after collection of all eggs (covered with intravenous Augmentin followed by 3 days of oral Azithromycin antibiotic) VERSUS no aspiration.

Criteria for surgical treatment prior to IVF - Ultrasound visible hydrosalpinges (uni- or bilateral) during ovarian stimulation phase of IVF treatment or severe tubal pathology.

Timing of intervention: All interventions administered just after oocyte aspiration of the IVF cycle studied.

Cointerventions: antibiotics were administered in the aspiration group.

Data on performer: not stated.

Outcomes PRIMARY OUTCOMES

Biochemical (urinary HCG test 14 days after ET) and clinical (transvaginal ultrasound verified gestation sac) pregnancy per woman.

\section{SECONDARY OUTCOMES}

Ectopic pregnancy rate - not defined; presumably per woman.

Miscarriage - any pregnancy loss before 12 weeks.

Implantation rate - number of gestational sacs on ultrasound divided by the number of embryos transferred.

Pelvic infection - pelvic abdominal pain and pyrexia or positive culture of genital swabs

Recurrence rate of hydrosalpinges after aspiration.

\section{Notes}

\section{Risk of bias}

\begin{tabular}{|c|c|c|}
\hline Bias & Authors' judgement & Support for judgement \\
\hline Allocation concealment? & Low risk & $\begin{array}{l}\text { Randomisation by computer algorithm using a third party administrator just } \\
\text { prior to oocyte retrieval procedure. }\end{array}$ \\
\hline $\begin{array}{l}\text { Blinding? } \\
\text { All outcomes. Partici- } \\
\text { pants, outcome assessors, } \\
\text { clinicians, statisticians }\end{array}$ & High risk & Investigator nor the outcome assessor were blinded. \\
\hline $\begin{array}{l}\text { Incomplete outcome data } \\
\text { addressed? } \\
\text { Intention to treat for pri- } \\
\text { mary outcome? }\end{array}$ & Low risk & 'Intention to treat' analysis performed (as there were no drop-outs or losses). \\
\hline $\begin{array}{l}\text { Free of selective report- } \\
\text { ing? }\end{array}$ & Low risk & No suggestion of selective reporting. \\
\hline Detection adequate? & Low risk & Follow-up duration up to 1 IVF cycle. \\
\hline Source of funding stated? & Low risk & No source of funding reported. \\
\hline $\begin{array}{l}\text { Powercalculation per- } \\
\text { formed? }\end{array}$ & High risk & $\begin{array}{l}\text { Power calculation performed - sample size } 158 \text { not attained owing to decrease } \\
\text { in recruitment rate by virtue of patient preference for salpingectomy, recruit- } \\
\text { ment was stopped on advice of the Data Monitoring Committee after } 4 \text { years. }\end{array}$ \\
\hline $\begin{array}{l}\text { Loss to Follow-up ex- } \\
\text { plained? }\end{array}$ & Low risk & Yes, no loss to follow-up occurred. \\
\hline
\end{tabular}




Randomised Controlled Trial
Study in two centres: a academic centre: Aretaieon University Hospital; a non-academic centre: the
Centre for Human Reproduction, Genesis Clinic; both in Athens, Greece. Patients recruited prospective-
ly; patient sampling consecutively.
Inclusion criteria: Women aged $\leq 41$ with unilateral of bilateral hydrosalpinges; confirmed by HSG, suit-
ability for IVF-ICSI treatment, FSH on day $2 / 3<12 \mathrm{mIU/ml,} \mathrm{no} \mathrm{contraindications} \mathrm{for} \mathrm{laparoscopic} \mathrm{surgery,}$
no history of IVF attempts before recruitment, absence of other obvious pelvic pathology in females.
Exclusion criteria: Not specified.

115 patients were recruited and randomised (50 to proximal tubal occlusion; 50 to salpingectomy; 15 to non-intervention). 112 patients underwent IVF and were analysed. 3 women withdrew from IVF; 3 women didn't respond to ovarian stimulation and in 4 women IVF did not result in embryo's.

Follow-up scheme: up to the first IVF cycle after tubal surgery. Type: ultrasound 4 weeks after ET.

\title{
Participants
}

Interventions

\author{
Age (years (SD)): $31 \pm 4.5$ vs $29.8 \pm 3.4$ vs $3.4 \pm 5.3$. \\ Type (primary or secondary) of subfertility: primary subfertility in $92 \%$ vs $84 \%$ vs $87 \%$ of the patients; \\ duration not stated. \\ Previous investigation: included baseline FSH. \\ Contributary causes for infertility: other obvious pathology was reason for exclusion. \\ Previous treatments: no previous fertility treatment.
}

Diagnosis of tubal pathology was confirmed with HSG.

Characteristics and distribution of tubal pathology: all patients had hydrosalpinges; bilateral in 70/115 patients ( $70 \%$ vs $54 \%$ vs $54 \%$ ). $70 / 115$ women ( $58 \%$ vs $64 \%$ vs $60 \%$ ) had ultrasound visible hydrosalpinges.

\section{Characteristics of IVF treatment:}

IVF protocol: a standard long antagonist protocol; ovarian stimulation; rec FSH; HCG; luteal phase support; ET on day 3. Doxycycline and prednisolon 6 days after oocyte retrieval.

Number of ovarian stimulation cycles per woman: one

Collected oocytes (mean \pm SD): $11.6 \pm 4.9$ in the laparoscopic occlusion group; $12.1 \pm 5.0$ in the salpingectomy group, $10.9 \pm 5.1$ in the non-intervention group. ICSI procedures: $n=7(14.9 \%)$ in the laparoscopic occlusion group; $n=6(12.5 \%)$ in the salpingectomy group, $n=3(20 \%)$ in the non-intervention group. Fertilized oocytes (mean \pm SD): $8.7 \pm 3.9$ in the laparoscopic occlusion group; $8.53 \pm 4.0$ in the salpingectomy group, $7.9 \pm 5.1$ in the non-intervention group. No of transferred embryo's: 118 in the laparoscopic occlusion group; 121 in the salpingectomy group, 36 in the non-intervention group.

Embryo's per ET (mean \pm SD) 2.6 \pm 0.6 in the laparoscopic occlusion group; $2.6 \pm 0.6$ in the salpingectomy group, $2.6 \pm 0.8$ in the non-intervention group. Number of transfers with all embryo's grade 2.7 in the laparoscopic occlusion group; 5 in the salpingectomy group, 1 in the non-intervention group.

Uni- or bilateral laparoscopic tubal occlusion by bipolar diathermy at two separate sites on the isthmic segment of the tube VERSUS uni- or bilateral salpingectomy; with transection 1 to $1.5 \mathrm{~cm}$ from the cornual section VERSUS no intervention.

Criteria for surgery: unilateral of bilateral hydrosalpinges.

Timing of surgery: 2 to 3 months after tubal surgery prior to IVF; time interval between randomisation to IVF in the control group:unclear.

co-interventions: none stated.

Data on performer: not stated.

Outcomes

PRIMARY OBJECTIVES

Ongoing pregnancy rate - (pregnancy's beyond first trimester) per $100 \mathrm{ET}$.

Clinical pregnancy rate - (gestational sacs on ultrasound with fetal pole, 4 weeks after ET) per $100 \mathrm{ET}$. Ectopic pregnancy rate - defined per 100 ET.

Miscarriage rate - abortion of clinical pregnancies (ultrasonographically identified pregnancies) per $100 \mathrm{ET}$. 
Kontoravdis 2006 (Continued)

SECONDARY OBJECTIVES:

Subgroup analysis for bilateral hydrosalpinges and ultrasound visible hydrosalpinges.

Notes

\section{Risk of bias}

\begin{tabular}{|c|c|c|}
\hline Bias & Authors' judgement & Support for judgement \\
\hline Allocation concealment? & Low risk & Randomisation by computer generated randomisation in blocks. \\
\hline $\begin{array}{l}\text { Blinding? } \\
\text { All outcomes. Partici- } \\
\text { pants, outcome assessors, } \\
\text { clinicians, statisticians }\end{array}$ & High risk & The operator and the IVF performer were the same person in some cases. \\
\hline $\begin{array}{l}\text { Incomplete outcome data } \\
\text { addressed? } \\
\text { Intention to treat for pri- } \\
\text { mary outcome? }\end{array}$ & High risk & $\begin{array}{l}112 \text { women analysed of } 115 \text { randomised. Intention to treat analysis not explic- } \\
\text { itly stated. }\end{array}$ \\
\hline $\begin{array}{l}\text { Free of selective report- } \\
\text { ing? }\end{array}$ & Low risk & No suggestions of selective outcome reporting. \\
\hline Detection adequate? & Low risk & adequate detection of stated outcomes. \\
\hline Source of funding stated? & Low risk & correspondence stated that there was no source of funding. \\
\hline $\begin{array}{l}\text { Powercalculation per- } \\
\text { formed? }\end{array}$ & Unclear risk & $\begin{array}{l}\text { Power calculation is performed and calculated sample sizes were achieved. } \\
\text { However, power calculations in this trial were made with very large anticipat- } \\
\text { ed differences ( } 46 \% \text { vs } 73 \% \text { comparing salpingectomy to tubal occlusion and } \\
46 \% \text { vs } 14 \% \text { comparing surgery to no surgery) and not performed correctly. } \\
\text { lt is quite unlikely that these anticipated differences would be achieved; and } \\
\text { therefore it may be questioned whether the made power calculation was ad- } \\
\text { hered to. }\end{array}$ \\
\hline $\begin{array}{l}\text { Loss to Follow-up ex- } \\
\text { plained? }\end{array}$ & Low risk & Withdrawal and losses to follow-up were explained. \\
\hline
\end{tabular}

Moshin 2006

$\begin{array}{ll}\text { Methods } & \text { Randomised Controlled Trial. } \\ & \text { Single academic centre, Center for Reproductive Health and Genetics, Chisinau, Moldova. } \\ & \text { Patients were recruited prospectively and consecutively. } \\ & \text { Inclusion criteria: women with hydrosalpinges. } \\ & \text { Exclusion criteria: not specified. } \\ & 204 \text { patients recruited and randomised (66 women randomised to no surgical treatment; } 60 \text { women } \\ \text { randomised to salpingectomy; } 78 \text { randomised to proximal tubal occlusion) } 204 \text { women analysed. loss } \\ \text { to follow-up/withdrawal: none. } \\ \text { Follow-up duration: up to one IVF cycle. } \\ \text { Type of follow-up: diagnosis of pregnancy by ultrasound examination } 21 \text { days and } 35 \text { days after ET. }\end{array}$


Type (primary or secondary): about $65 \%$ of the patients in each group were primary infertile.

Duration of infertility: >2 years (mean months (SD): salpingectomy group: 46.2 (27.2); tubal occlusion group: 47.4 (28.5); non-intervention group: 46.9 (27.8)).

Previous investigative work-up: ovulatory cycles, hormonal tests, transvaginal ultrasound examination of the genitalia interna, male semen parameters.

Contributary causes to infertility: none.

Previous fertility treatment: no previous IVF treatment.

Diagnosis of tubal pathology: bilateral distal tubal occlusion diagnosed by HSG or ultrasound visible hydrosalpinges.

Characteristics and distribution of tubal pathology: bilateral hydrosalpinges.

IVF:

Ovarian stimulation in a long course GnRH analogue protocol (Decapeptyl or Dipherelin) with a fixed doses (225 IU daily) of recombinant FSH for stimulation, starting on day 3 of the cycle. Ovarian response was monitored by ultrasonography and serum E2 concentration. Ovulation was triggered with 10000 IU of HCG (Pregnyl) when the leading follicles reached $18-20 \mathrm{~mm}$. Oocyte retrieval was carried out 36 hours after HCG administration. Retrieved oocytes were evaluated and fertilized by conventional insemination. Embryos were transferred on day 3 after insemination. : Utrogestan $400 \mathrm{mg} /$ day vaginal from the day of ovum pick-up until 12 week of pregnancy. Results reported over one cycle. Number of oocytes retrieved: $9.8 \pm 5.5$ in the non-intervention group / 10.4 \pm 6.0 in the salpingectomy group, $10.2 \pm 5.7$ in the proximal occlusion group.

Proportion undergoing ICSI: not stated. Fertilized oocytes: $6.8 \pm 4.6$ in the non-intervention group,

$7.0 \pm 4.7$ in the salpingectomy group, $6.9 \pm 4.6$ in the proximal occlusion group.

Mean number of preembryo's transferred: $3.5 \pm 1.3$ in the non-intervention group, $3.4 \pm 1.2$ in the salpingectomy group, $3.4 \pm 1.3$ in the proximal occlusion group.

Criterium for intervention: ultrasound visible hydrosalpinges.

Timing of intervention: minimally 2 months before IVF treatment. Timing to the IVF treatment cycle after randomisation in the control group: unclear.

Cointerventions: not reported.

Data on performer: not reported.

Outcomes
PRIMARY OUTCOMES:
Clinical pregnancy - gestational sac on ultrasound.

\section{Risk of bias}

\begin{tabular}{lll}
\hline Bias & Authors' judgement & Support for judgement \\
\hline Allocation concealment? & Low risk & By opaque numbered envelopes \\
\hline $\begin{array}{l}\text { Blinding? } \\
\begin{array}{l}\text { All outcomes. Partici- } \\
\text { pants, outcome assessors, } \\
\text { clinicians, statisticians }\end{array}\end{array}$ & Unclear risk & Not stated. \\
\hline $\begin{array}{l}\text { Incomplete outcome data } \\
\text { addressed? } \\
\text { Intention to treat for pri- } \\
\text { mary outcome? }\end{array}$ & Low risk & $\begin{array}{l}\text { Not stated in abstract; but number of patients randomised is the same as } \\
\text { numbers of patients analysed, correspondence clarified there was no loss to } \\
\text { follow-up or withdrawal. }\end{array}$ \\
\hline
\end{tabular}


Moshin 2006 (Continued)
Free of selective report-
Low risk
no suggestions of selective reporting. ing?

\begin{tabular}{lll}
\hline Detection adequate? & Low risk & yes, adequate follow-up after IVF. \\
\hline Source of funding stated? & Low risk & $\begin{array}{l}\text { Source of funding: the Academy of Science of the Republic of Moldova (clari- } \\
\text { fied by correspondence) }\end{array}$ \\
\hline $\begin{array}{l}\text { Powercalculation per- } \\
\text { formed? }\end{array}$ & Unclear risk & $\begin{array}{l}\text { Not clear. As study sizes were small it may be presumed that they have not } \\
\text { been adhered to if they where performed. }\end{array}$ \\
\hline $\begin{array}{l}\text { Loss to Follow-up ex- } \\
\text { plained? }\end{array}$ & Low risk & No loss to Follow-up/ withdrawal, clarified by correspondence. \\
\hline
\end{tabular}

\section{Strandell 1999}

Methods Randomised controlled trial.

Multicentre trial involving 9 Scandinavian IVF centres.

Patients recruited prospectively; patient sampling not explained explicitly.

Inclusion criteria: hydrosalpinges, laparoscopic acces ability, age $<39$ at time of randomisation.

Exclusion criteria - previous IVF; uterine fibroids.Concomitant male factor requiring ICSI accepted if

centre had established successful ICSI programme with results equivalent to conventional IVF.

204 women were recruited and randomised. Number of exclusions not known. 5 patients of the intervention group did not undergo surgery; 6 patients in the intervention group and 6 patients in the nonintervention group did not undergo IVF. 204 women were analysed.

Follow-up duration up to 1 IVF cycle (further follow-up data anticipated).

\section{Participants}

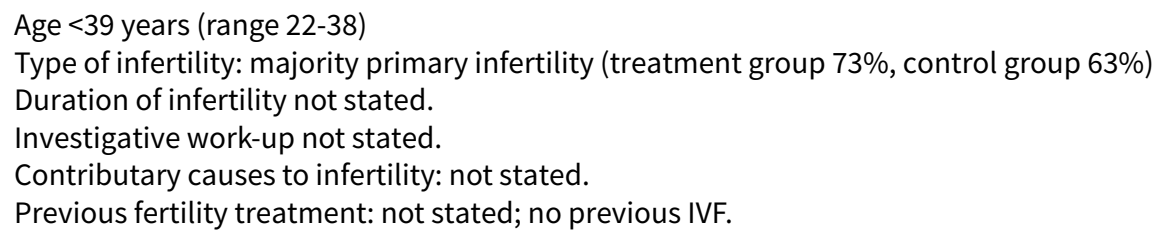

Characteristics and distribution of tubal pathology: Hydrosalpinges. Bilateral in 69(59\%) and 36(41\%); unilateral in $47(41 \%)$ and 52(59\%).

Characteristics of IVF treatment :

IVF protocol: Long GnRh antagonist protocol, with either HMG of FSH.

1-2 IVF ovarian stimulation cycles per woman.

Mean oocytes retrieved at IVF (SD) - 10.6 (5.9) in the salpingectomy group; 10.6 (6.1) in the non intervention group. Proportion undergoing ICSI, $13.1 \%$ in the salpingectomy group; in the non intervention group $12.6 \%$. Mean no. of fertilized and cleaved oocytes in treatment group 6.8 (4.8); 7.0 (4.9) in the non intervention group. Mean embryos transferred per cycles (SD) - treatment group $2.0(0.3) ; 2.0(0.4)$ in the non intervention group. proximal ligation and distal fenestration recommended) VERSUS no surgery.

Criteria for surgical treatment prior to IVF - Uni- or bilateral hydrosalpinges (a distally occluded pathologically dilated tube or one which became pathologically dilated on patency testing by HSG/laparoscopy. 
Strandell 1999 (Continued)

Interval from surgical intervention to IVF - minimum 2 months, IVF was performed in the control group

directly after randomisation.

Co-interventions: none (deviations from protocol though)

Data on surgeons: not stated.

PRIMARY OUTCOMES
Ongoing pregnancy (pregnancy>20 weeks) or delivery rate in first cycle per woman, per started cycle,
and per transfer cycle.
Pregnancy rate - per woman, per started cycle and per transfer cycle.
Clinical pregnancy verified by gestational sac on ultrasound.
SECONDARY OUTCOMES
Ectopic pregnancy rate - per implanted embryo's/ clinical pregnancy
Miscarriage rate - not defined, presumably as outcome of clinical pregnancy
Implantation rate - number of gestational sacs on ultrasound divided by the number of embryos trans-
ferred.
Multiple pregnancy rate not stated

Notes

\section{Risk of bias}

\begin{tabular}{|c|c|c|}
\hline Bias & Authors' judgement & Support for judgement \\
\hline Allocation concealment? & Low risk & True randomisation - sealed opaque envelopes in blocks of $10-30$. \\
\hline $\begin{array}{l}\text { Blinding? } \\
\text { All outcomes. Partici- } \\
\text { pants, outcome assessors, } \\
\text { clinicians, statisticians }\end{array}$ & High risk & No blinding. \\
\hline $\begin{array}{l}\text { Incomplete outcome data } \\
\text { addressed? } \\
\text { Intention to treat for pri- } \\
\text { mary outcome? }\end{array}$ & Low risk & 'Intention to treat' analysis performed. \\
\hline $\begin{array}{l}\text { Free of selective report- } \\
\text { ing? }\end{array}$ & Low risk & No suggestions of selective reporting. \\
\hline Detection adequate? & Low risk & Follow-up duration up to 1 IVF cycle (further follow-up data anticipated). \\
\hline Source of funding stated? & Low risk & $\begin{array}{l}\text { Study was funded by grants from the Goteborg Medical Society, the Hjalmar } \\
\text { Svensson Foundation and the society 'ordensallskapet W: } 6 \text { ' }\end{array}$ \\
\hline $\begin{array}{l}\text { Powercalculation per- } \\
\text { formed? }\end{array}$ & High risk & $\begin{array}{l}\text { Power calculation performed - sample size } 300 \text { not adhered to due to decrease } \\
\text { in recruitment rate. }\end{array}$ \\
\hline $\begin{array}{l}\text { Loss to Follow-up ex- } \\
\text { plained? }\end{array}$ & Low risk & Withdrawal and loss to follow up explained. \\
\hline
\end{tabular}

Characteristics of excluded studies [ordered by study ID]

\begin{tabular}{ll}
\hline Study & Reason for exclusion \\
\hline Gelbaya 2006 & Retrospective non-randomised controlled study. \\
\hline
\end{tabular}




\begin{tabular}{ll}
\hline Study & Reason for exclusion \\
\hline Goldstein 1998 & $\begin{array}{l}\text { Study design and whether randomisation has been performed is unclear. It appears that patients } \\
\text { serve as there own control after surgical intervention. Correspondance was sought to resolve this } \\
\text { issue, but not with success. }\end{array}$
\end{tabular}

Hotineanu 2007

This trial was excluded because this article compared proximal/distal tubal occlusion with clamping; however comparisons were between outcomes in different degrees of tubal pathology rather than different interventions. Comparative data was not obtained as correspondence was unsuccessful.

\section{Mardesic 1999}

This trial is not a randomised trial - the 'control' group was historical (the same women prior to their salpingectomy) and biased by the fact that no pregnancies occurred. formed after tubal surgery.

\section{Characteristics of ongoing studies [ordered by study ID]}

Darwish 2005,2006, 2007

Trial name or title
Is there a role for hysteroscopic tubal occlusion of functionless hydrosalpinges prior to IVF/ICSI in modern practice?

\section{Randomised controlled trial.}

Single academic centre in Egypt (women's Health University Center, Assiut University, Assiut Egypt) Patient recruitment from April 2004 to October 2006.

Inclusion criteria: women with uni- or bilaterally laparoscopically-proven functionless hydrosalpinges (a large blocked tube with lost major and minor folds) scheduled for IVF.

Exclusion criteria: women with a frozen pelvis at laparoscopy or inaccessible tubes at lap or hysteroscopy.

Follow-up: HSG in the following cycle after the procedure; follow-up procedure for pregnancy unclear.

\section{Participants}

Women with uni- or bilaterally laparoscopically-proven functionless hydrosalpinges scheduled for IVF. Women with a frozen pelvis at laparoscopy or inaccessible tubes at lap or hysteroscopy were excluded.

\begin{tabular}{ll}
\hline Interventions & $\begin{array}{l}\text { Laparoscopic occlusion by } \\
\text { VERSUS Hysteroscopic tuba } \\
\text { duced by a resectoscope; a } \\
\text { Procedures are performed }\end{array}$ \\
\hline Outcomes & PRIMARY OUTCOMES: \\
& Feasible acces \\
Confirmed tubal occlusion \\
SECONDARY OUTCOMES \\
Operative time \\
Hospital stay \\
Complications \\
Pregnancy: not defined.
\end{tabular}

April 2004 
Darwish 2005,2006, 2007 (Continued)

Contact information

Notes
Atef M.M. Darwish, dept of obstetrics and gynaecology, women's Health University, P.O. Box(1), 71111 Assiut Egypt. a_darwish@mailcity.com

The objective of Darwish 2007 is mainly to conduct a safety study and a study to address the value of hysteroscopic tubal occlusion compared to laparoscopic tubal occlusion. This current study is stated to be only a preliminary study of which the follow-up time was to short to detect pregnancy. Subsequent studies might report outcomes in terms of pregnancy.

\section{DATA AND ANALYSES}

Comparison 1. Laparoscopic surgery on the fallopian tube (all types) VERSUS No surgery on the fallopian tube (all types)

\begin{tabular}{|c|c|c|c|c|}
\hline Outcome or subgroup title & $\begin{array}{l}\text { No. of } \\
\text { studies }\end{array}$ & $\begin{array}{l}\text { No. of } \\
\text { partici- } \\
\text { pants }\end{array}$ & Statistical method & Effect size \\
\hline 1 Ongoing pregnancy rate & 3 & 329 & Odds Ratio (M-H, Fixed, 95\% Cl) & $2.20[1.26,3.82]$ \\
\hline $\begin{array}{l}1.1 \text { Laparoscopic bilateral salpingecto- } \\
\text { my and adhesiolysis versus no surgery } \\
\text { on the fallopian tube }\end{array}$ & 1 & 60 & Odds Ratio (M-H, Fixed, 95\% Cl) & $3.06[0.97,9.66]$ \\
\hline $\begin{array}{l}\text { 1.2 Laparoscopic salpingectomy versus } \\
\text { no intervention }\end{array}$ & 2 & 269 & Odds Ratio (M-H, Fixed, 95\% Cl) & $1.99[1.06,3.74]$ \\
\hline 2 Clinical pregnancy rate & 3 & 395 & Peto Odds Ratio (Peto, Fixed, 95\% Cl) & $2.31[1.48,3.62]$ \\
\hline $\begin{array}{l}2.1 \text { Laparoscopic salpingectomy versus } \\
\text { no intervention }\end{array}$ & 3 & 395 & Peto Odds Ratio (Peto, Fixed, 95\% Cl) & $2.31[1.48,3.62]$ \\
\hline 3 Pregnancy rate - any definition & 4 & 455 & Odds Ratio (M-H, Fixed, 95\% Cl) & $2.49[1.60,3.86]$ \\
\hline $\begin{array}{l}\text { 3.1 Laparoscopic bilateral salpingecto- } \\
\text { my and adhesiolysis versus no surgery } \\
\text { on the fallopian tube }\end{array}$ & 1 & 60 & Odds Ratio (M-H, Fixed, 95\% Cl) & $3.06[0.97,9.66]$ \\
\hline $\begin{array}{l}\text { 3.2 Laparoscopic salpingectomy versus } \\
\text { no intervention }\end{array}$ & 3 & 395 & Odds Ratio (M-H, Fixed, 95\% Cl) & $2.40[1.49,3.86]$ \\
\hline 4 Ectopic pregnancy rate & 3 & 329 & Peto Odds Ratio (Peto, Fixed, 95\% Cl) & $0.68[0.13,3.56]$ \\
\hline $\begin{array}{l}\text { 4.1 Laparoscopic bilateral salpingecto- } \\
\text { my and adhesiolysis versus laparoscop- } \\
\text { ic adhesiolysis }\end{array}$ & 1 & 60 & Peto Odds Ratio (Peto, Fixed, 95\% Cl) & $0.14[0.00,6.82]$ \\
\hline $\begin{array}{l}4.2 \text { Laparoscopic salpingectomy versus } \\
\text { no intervention }\end{array}$ & 2 & 269 & Peto Odds Ratio (Peto, Fixed, 95\% Cl) & $0.96[0.15,6.01]$ \\
\hline 5 Miscarriage rate & 3 & 115 & Peto Odds Ratio (Peto, Fixed, 95\% Cl) & $0.38[0.11,1.28]$ \\
\hline
\end{tabular}




\begin{tabular}{lllll}
\hline Outcome or subgroup title & $\begin{array}{l}\text { No. of } \\
\text { studies }\end{array}$ & $\begin{array}{l}\text { No. of } \\
\text { partici- } \\
\text { pants }\end{array}$ & Statistical method & Effect size \\
\hline $\begin{array}{l}5.1 \text { Laparoscopic bilateral salpingecto- } \\
\text { my and adhesiolysis versus laparoscop- } \\
\text { ic adhesiolysis }\end{array}$ & 1 & 38 & Peto Odds Ratio (Peto, Fixed, 95\% Cl) & 0.17 [0.01, 5.24] \\
\hline $\begin{array}{l}5.2 \text { Laparoscopic salpingectomy versus } \\
\text { no intervention }\end{array}$ & 2 & 77 & Peto Odds Ratio (Peto, Fixed, 95\% Cl) & $0.42[0.12,1.57]$ \\
\hline $\begin{array}{l}6 \text { Surgical complication rate } \\
\text { 6.1 Laparoscopic salpingectomy versus } \\
\text { no intervention }\end{array}$ & 1 & 204 & Peto Odds Ratio (Peto, Fixed, 95\% Cl) & 5.86 [0.35, 96.79] \\
\hline
\end{tabular}

Analysis 1.1. Comparison 1 Laparoscopic surgery on the fallopian tube (all types) VERSUS No surgery on the fallopian tube (all types), Outcome 1 Ongoing pregnancy rate.

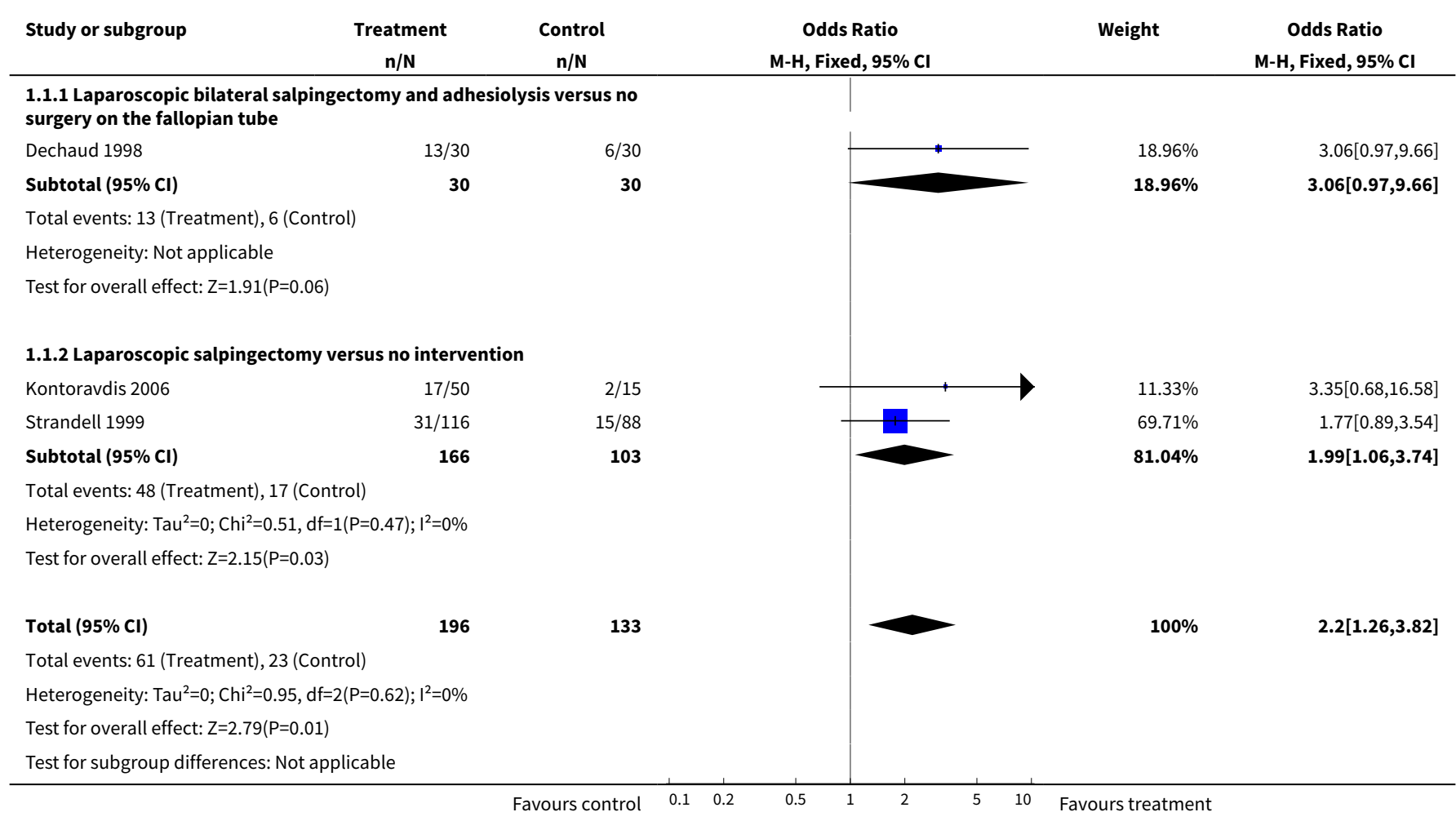

Analysis 1.2. Comparison 1 Laparoscopic surgery on the fallopian tube (all types) VERSUS No surgery on the fallopian tube (all types), Outcome 2 Clinical pregnancy rate.

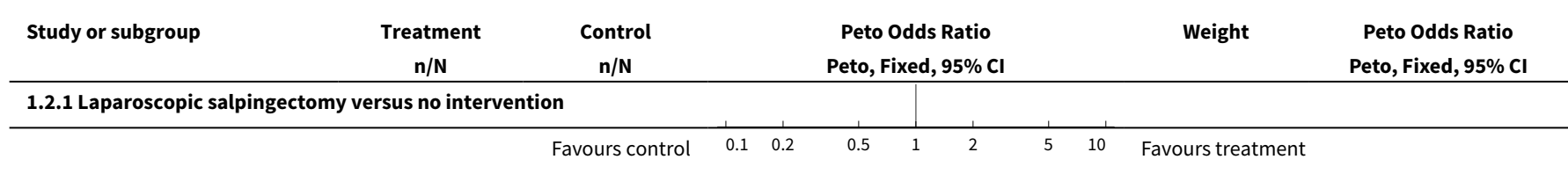




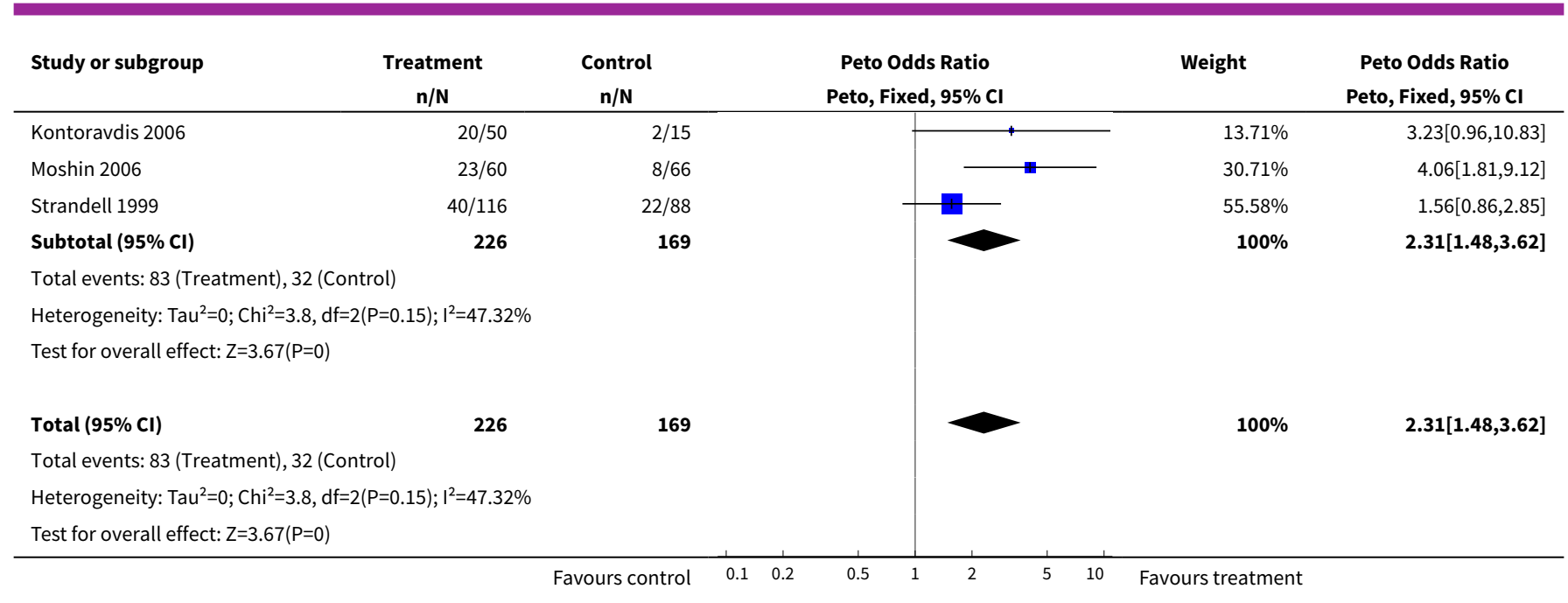

\section{Analysis 1.3. Comparison 1 Laparoscopic surgery on the fallopian tube (all types) VERSUS No surgery on the fallopian tube (all types), Outcome 3 Pregnancy rate - any definition.}

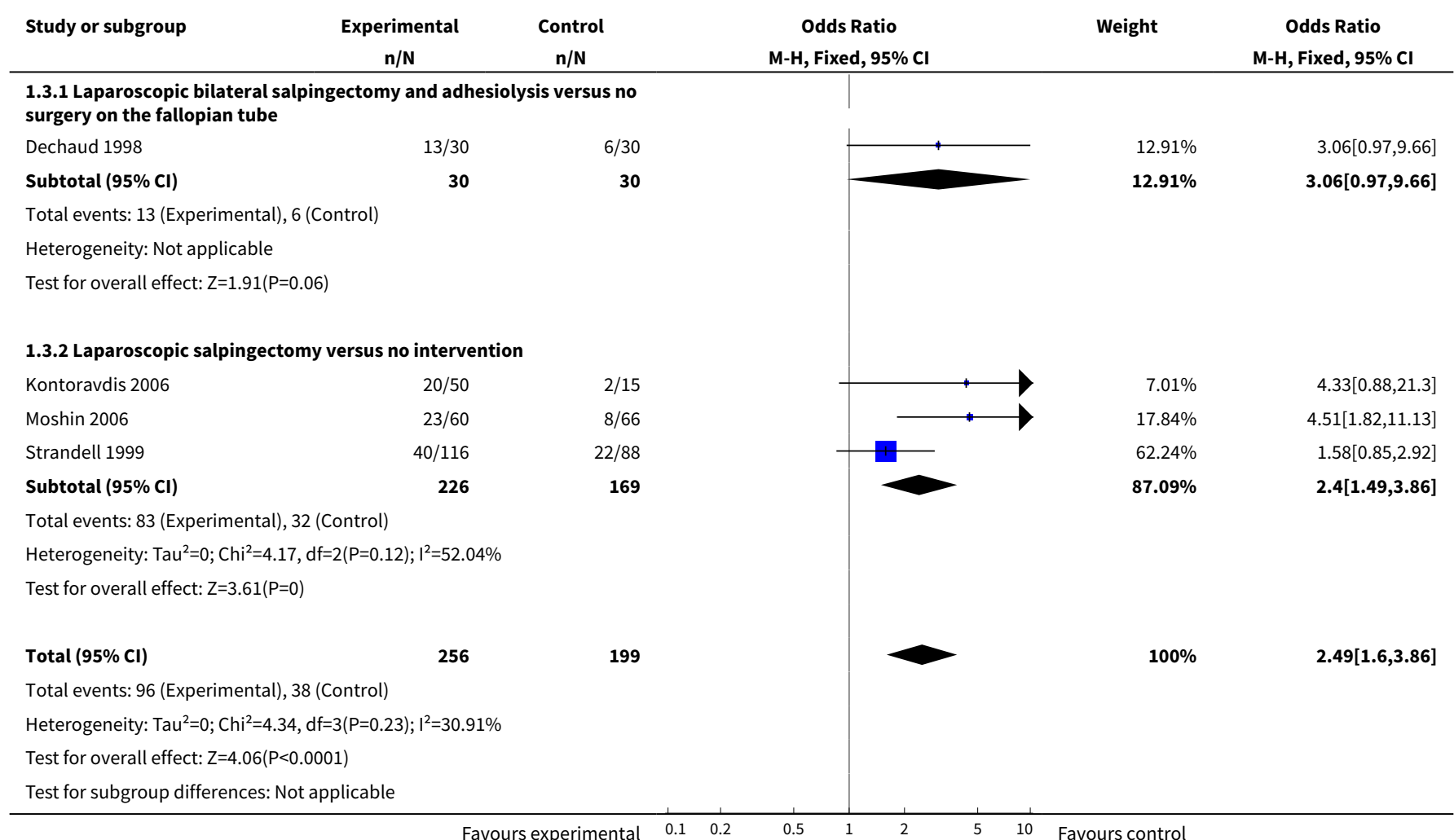


Analysis 1.4. Comparison 1 Laparoscopic surgery on the fallopian tube (all types) VERSUS No surgery on the fallopian tube (all types), Outcome 4 Ectopic pregnancy rate.

\begin{tabular}{|c|c|c|c|c|c|}
\hline Study or subgroup & $\begin{array}{c}\text { Treatment } \\
\mathrm{n} / \mathrm{N}\end{array}$ & $\begin{array}{c}\text { Control } \\
n / N\end{array}$ & $\begin{array}{c}\text { Peto Odds Ratio } \\
\text { Peto, Fixed, } 95 \% \mathrm{Cl}\end{array}$ & Weight & $\begin{array}{c}\text { Peto Odds Ratio } \\
\text { Peto, Fixed, } 95 \% \mathrm{Cl}\end{array}$ \\
\hline $\begin{array}{l}\text { 1.4.1 Laparoscopic } \\
\text { paroscopic adhesio }\end{array}$ & ctomy and adh & sis versus la- & & & \\
\hline Dechaud 1998 & $0 / 30$ & $1 / 30$ & & $17.93 \%$ & $0.14[0,6.82]$ \\
\hline Subtotal $(95 \% \mathrm{CI})$ & 30 & 30 & & $17.93 \%$ & $0.14[0,6.82]$ \\
\hline Total events: 0 (Trea & & & & & \\
\hline Heterogeneity: Not & & & & & \\
\hline Test for overall effec & & & & & \\
\hline 1.4.2 Laparoscopic & ersus no interve & & & & \\
\hline Kontoravdis 2006 & $1 / 50$ & $0 / 15$ & $t_{1}$ & $12.73 \%$ & $3.67[0.04,384.48]$ \\
\hline Strandell 1999 & $2 / 116$ & $2 / 88$ & & $69.34 \%$ & $0.75[0.1,5.53]$ \\
\hline Subtotal $(95 \% \mathrm{Cl})$ & 166 & 103 & & $82.07 \%$ & $0.96[0.15,6.01]$ \\
\hline Total events: 3 (Trea & & & & & \\
\hline Heterogeneity: Tau ${ }^{2}$ & $(P=0.54) ; I^{2}=0 \%$ & & & & \\
\hline Test for overall effec & & & & & \\
\hline Total $(95 \% \mathrm{Cl})$ & 196 & 133 & & $100 \%$ & $0.68[0.13,3.56]$ \\
\hline Total events: 3 (Trea & & & & & \\
\hline Heterogeneity: $\operatorname{Tau}^{2}$ & $(P=0.56) ; I^{2}=0 \%$ & & & & \\
\hline Test for overall effec & & & & & \\
\hline Test for subgroup di &,$d f=1(P=0.37)$ & & & & \\
\hline
\end{tabular}

Analysis 1.5. Comparison 1 Laparoscopic surgery on the fallopian tube (all types) VERSUS No surgery on the fallopian tube (all types), Outcome 5 Miscarriage rate.

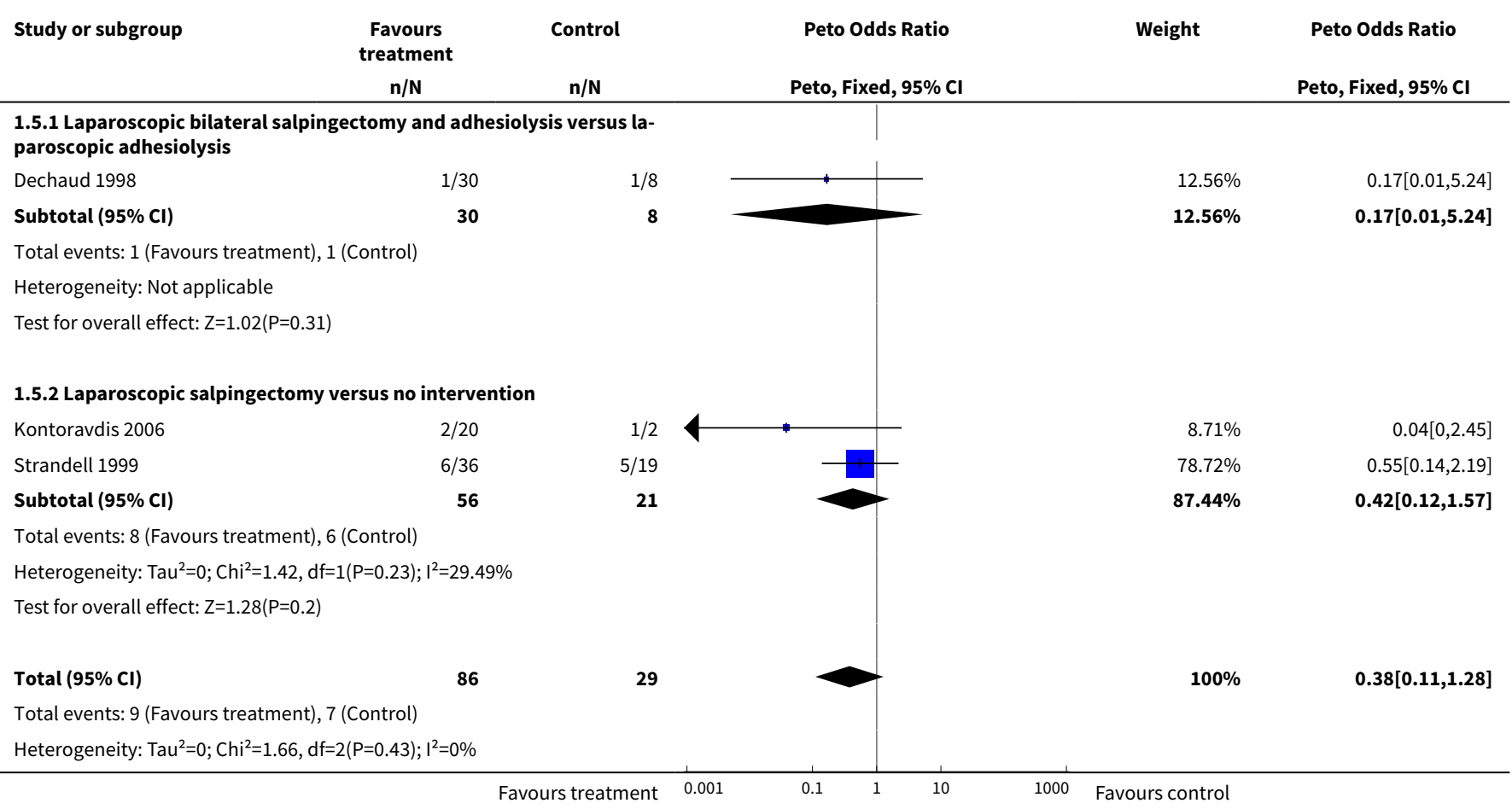




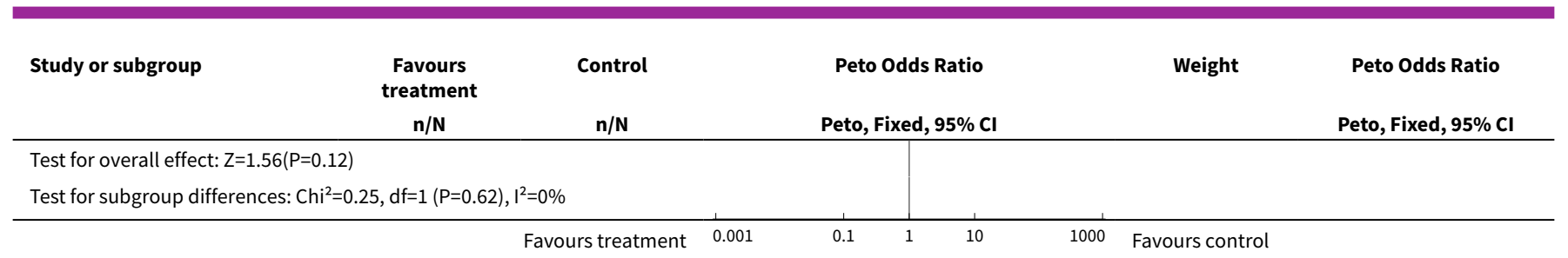

\section{Analysis 1.6. Comparison 1 Laparoscopic surgery on the fallopian tube (all types) VERSUS No surgery on the fallopian tube (all types), Outcome 6 Surgical complication rate.}

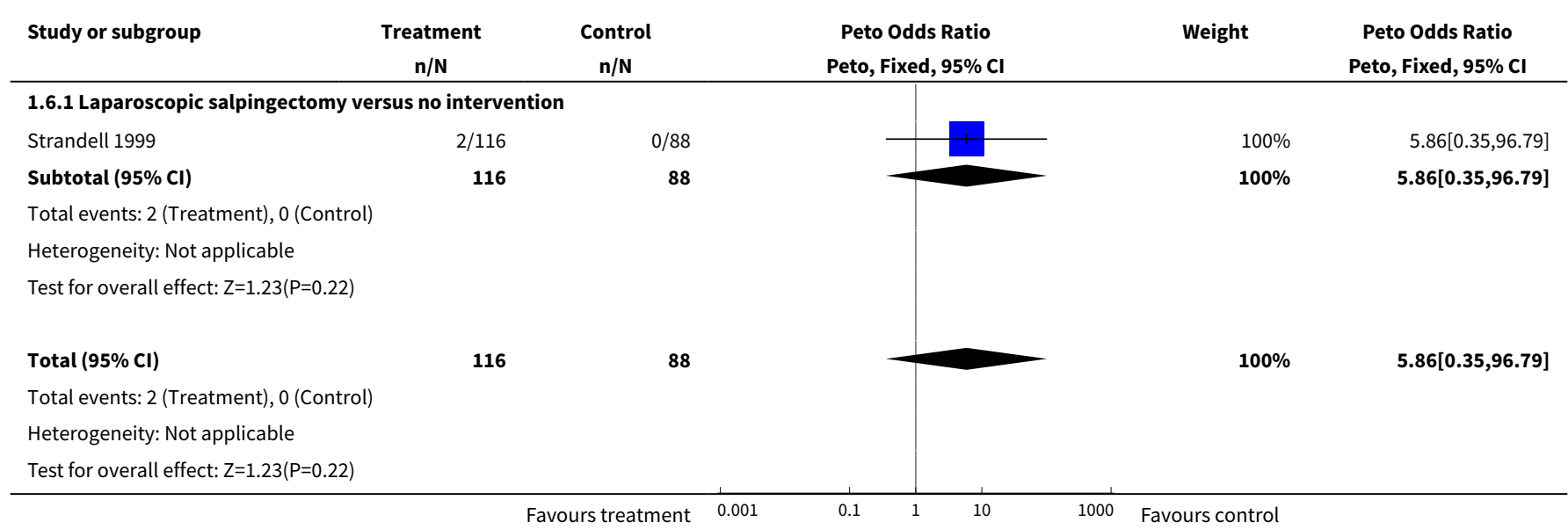

\section{Comparison 2. Occlusion of the fallopian tube VERSUS no intervention on the fallopian tube}

\begin{tabular}{|c|c|c|c|c|}
\hline Outcome or subgroup title & $\begin{array}{l}\text { No. of } \\
\text { studies }\end{array}$ & $\begin{array}{l}\text { No. of } \\
\text { partici- } \\
\text { pants }\end{array}$ & Statistical method & Effect size \\
\hline 1 Ongoing pregnancy rate & 1 & 65 & Odds Ratio (M-H, Fixed, 95\% Cl) & $7.21[0.87,59.57]$ \\
\hline $\begin{array}{l}\text { 1.1 Laparoscopic proximal tubal occlusion } \\
\text { by diathermy VERSUS no intervention }\end{array}$ & 1 & 65 & Odds Ratio (M-H, Fixed, 95\% Cl) & $7.21[0.87,59.57]$ \\
\hline 2 Clinical pregnancy rate & 2 & 209 & Odds Ratio (M-H, Fixed, 95\% Cl) & $4.66[2.17,10.01]$ \\
\hline $\begin{array}{l}2.1 \text { Laparoscopic proximal tubal occlusion } \\
\text { by diathermy VERSUS no intervention }\end{array}$ & 1 & 65 & Odds Ratio (M-H, Fixed, 95\% Cl) & $4.33[0.88,21.30]$ \\
\hline $\begin{array}{l}\text { 2.2 Proximal tubal clamping VERSUS no in- } \\
\text { tervention }\end{array}$ & 1 & 144 & Odds Ratio (M-H, Fixed, 95\% Cl) & $4.78[2.01,11.38]$ \\
\hline 3 Pregnancy rate - any definition & 2 & 209 & Odds Ratio (M-H, Fixed, 95\% Cl) & $4.66[2.17,10.01]$ \\
\hline $\begin{array}{l}3.1 \text { Laparoscopic proximal tubal occlusion } \\
\text { by diathermy VERSUS no intervention }\end{array}$ & 1 & 65 & Odds Ratio (M-H, Fixed, 95\% Cl) & $4.33[0.88,21.30]$ \\
\hline $\begin{array}{l}\text { 3.2 Laparoscopic proximal tubal clamping } \\
\text { VERSUS no intervention }\end{array}$ & 1 & 144 & Odds Ratio (M-H, Fixed, 95\% Cl) & $4.78[2.01,11.38]$ \\
\hline
\end{tabular}




\begin{tabular}{llllll}
\hline Outcome or subgroup title & $\begin{array}{l}\text { No. of } \\
\text { studies }\end{array}$ & $\begin{array}{l}\text { No. of } \\
\text { partici- } \\
\text { pants }\end{array}$ & Statistical method & Effect size \\
\hline 4 Ectopic pregnancy rate & 1 & 65 & Odds Ratio (M-H, Fixed, 95\% Cl) & $0.94[0.04,24.25]$ \\
\hline $\begin{array}{l}\text { 4.1 Laparoscopic proximal tubal occlusion } \\
\text { by diathermy VERSUS no intervention }\end{array}$ & 1 & 65 & Odds Ratio (M-H, Fixed, 95\% Cl) & $0.94[0.04,24.25]$ \\
\hline $\begin{array}{l}5 \text { Miscarriage rate } \\
\text { 5.1 Laparoscopic proximal tubal occlusion } \\
\text { by diathermy VERSUS no intervention }\end{array}$ & 1 & 25 & Odds Ratio (M-H, Fixed, 95\% Cl) & $0.15[0.01,3.09]$ \\
\hline
\end{tabular}

Analysis 2.1. Comparison 2 Occlusion of the fallopian tube VERSUS no intervention on the fallopian tube, Outcome 1 Ongoing pregnancy rate.

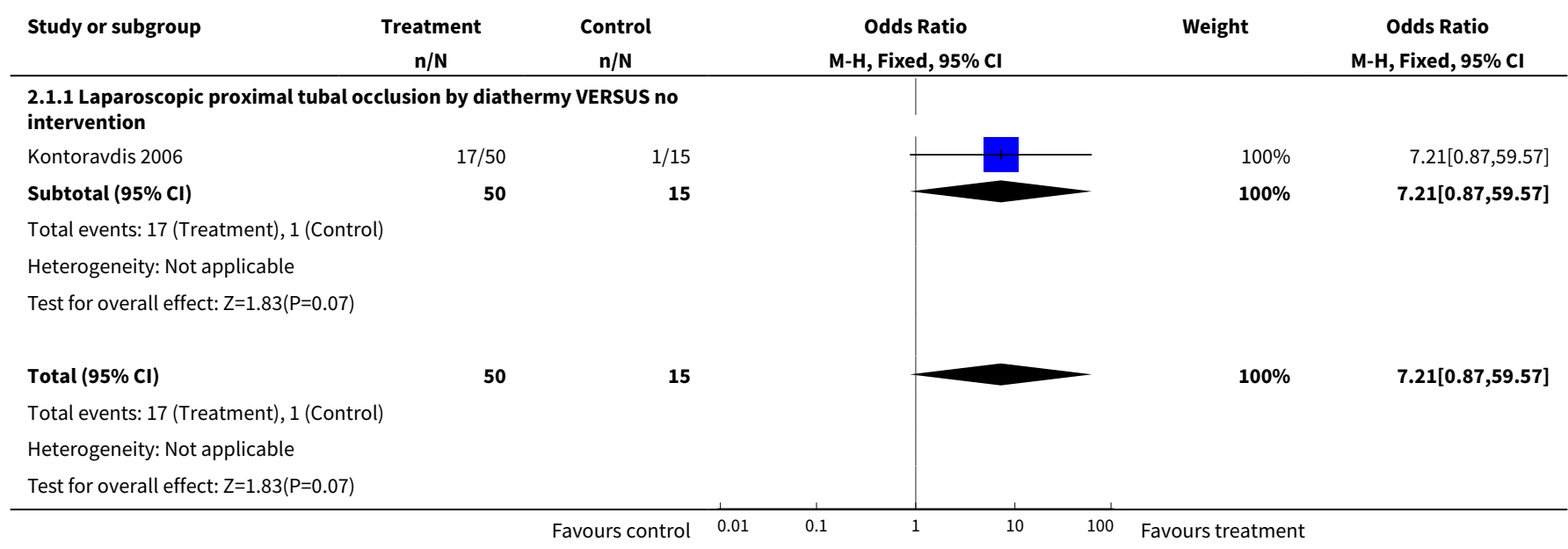

Analysis 2.2. Comparison 2 Occlusion of the fallopian tube VERSUS no intervention on the fallopian tube, Outcome 2 Clinical pregnancy rate.

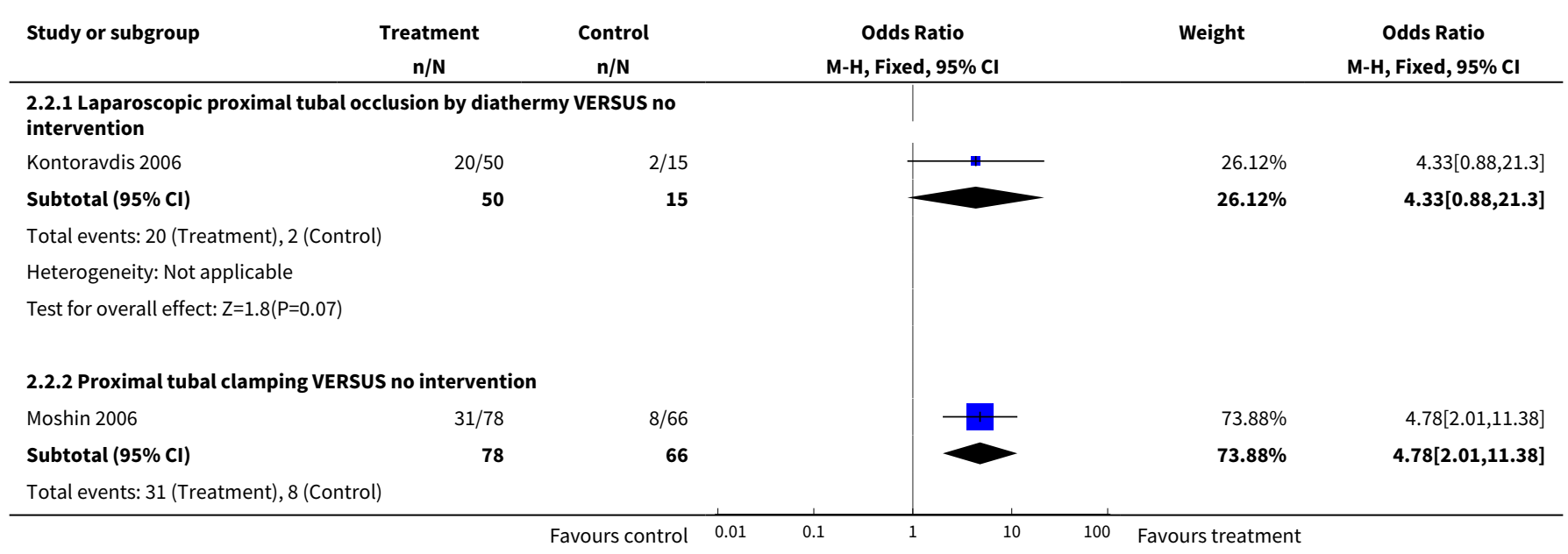




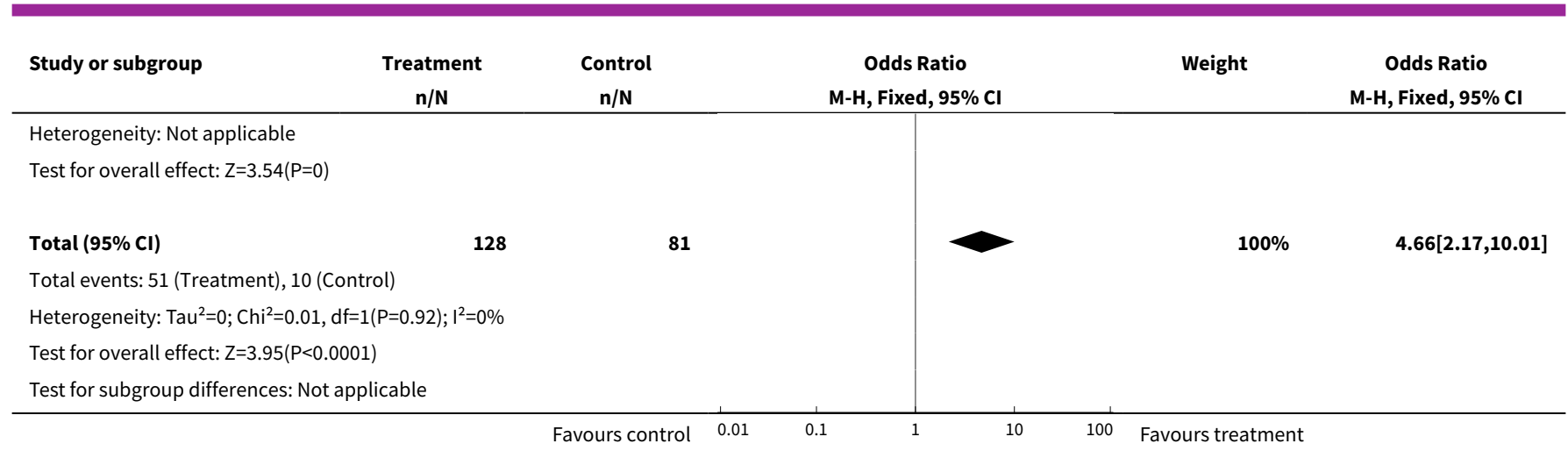

Analysis 2.3. Comparison 2 Occlusion of the fallopian tube VERSUS no intervention on the fallopian tube, Outcome 3 Pregnancy rate - any definition.

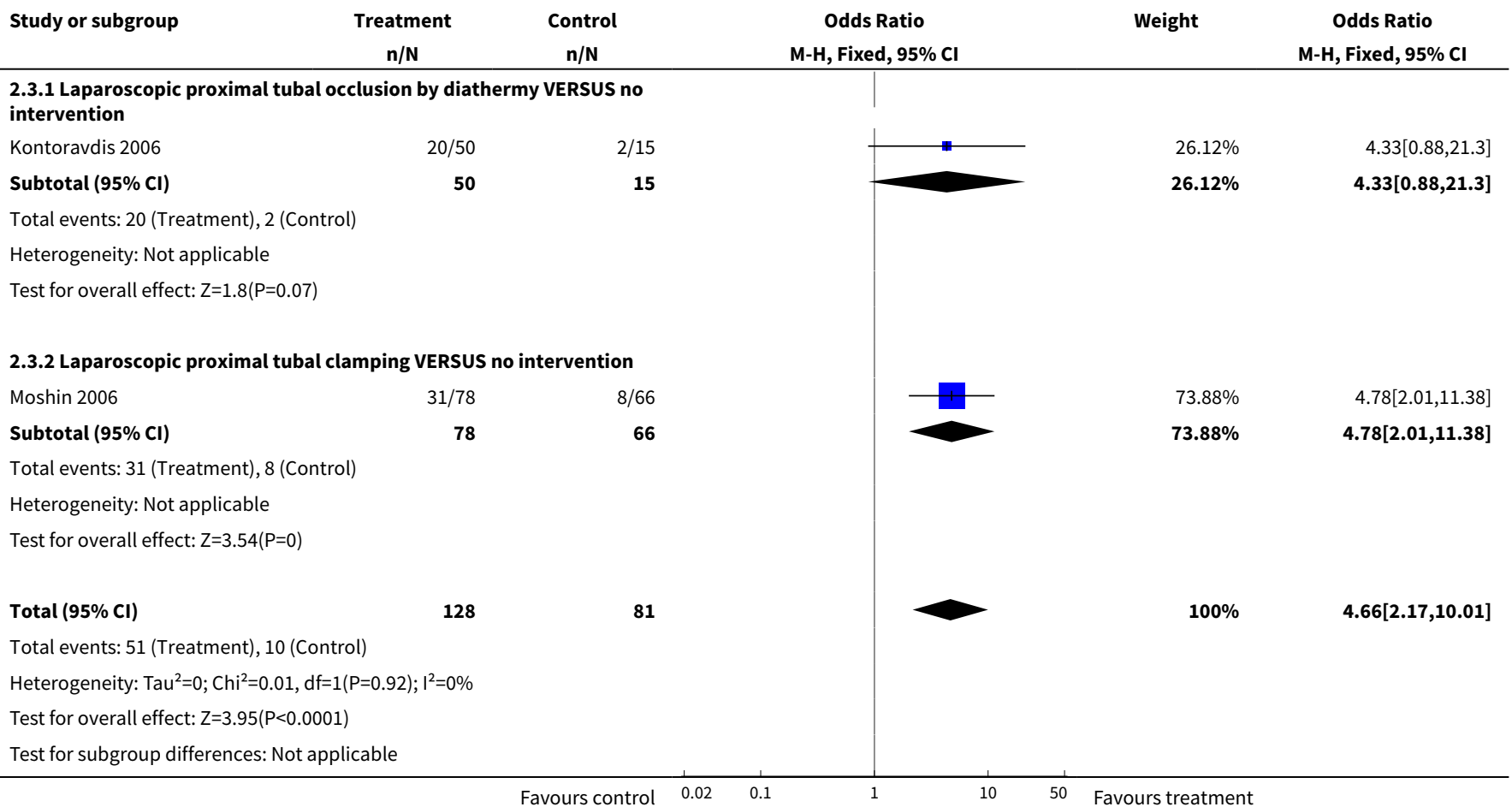

Analysis 2.4. Comparison 2 Occlusion of the fallopian tube VERSUS no intervention on the fallopian tube, Outcome 4 Ectopic pregnancy rate.

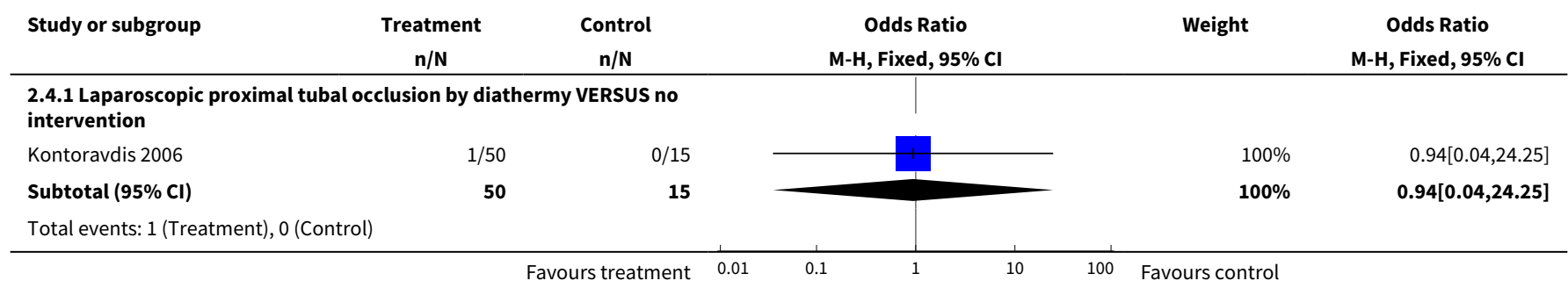




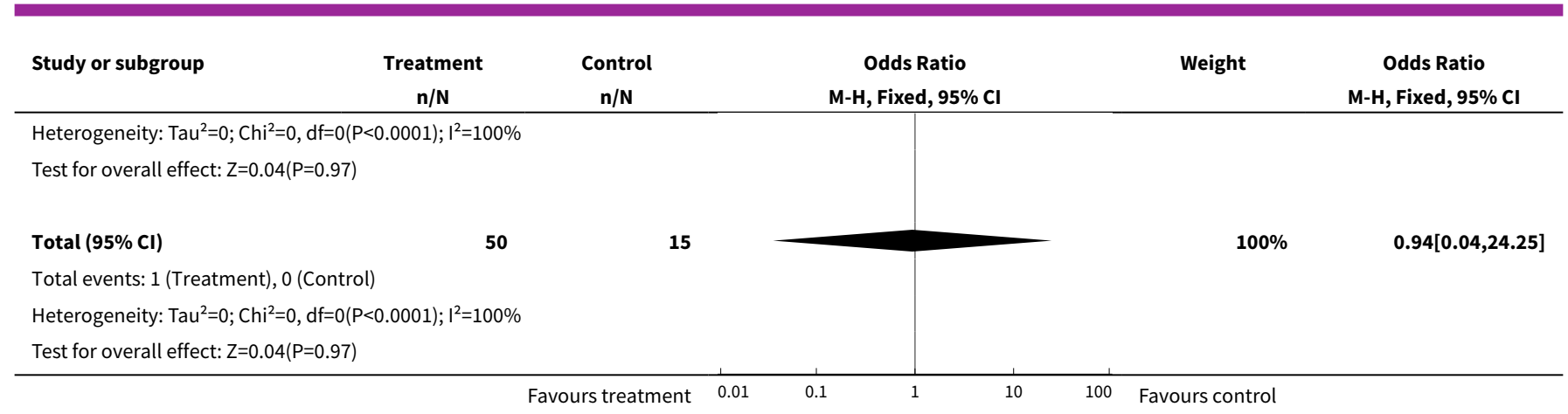

Analysis 2.5. Comparison 2 Occlusion of the fallopian tube VERSUS no intervention on the fallopian tube, Outcome 5 Miscarriage rate.

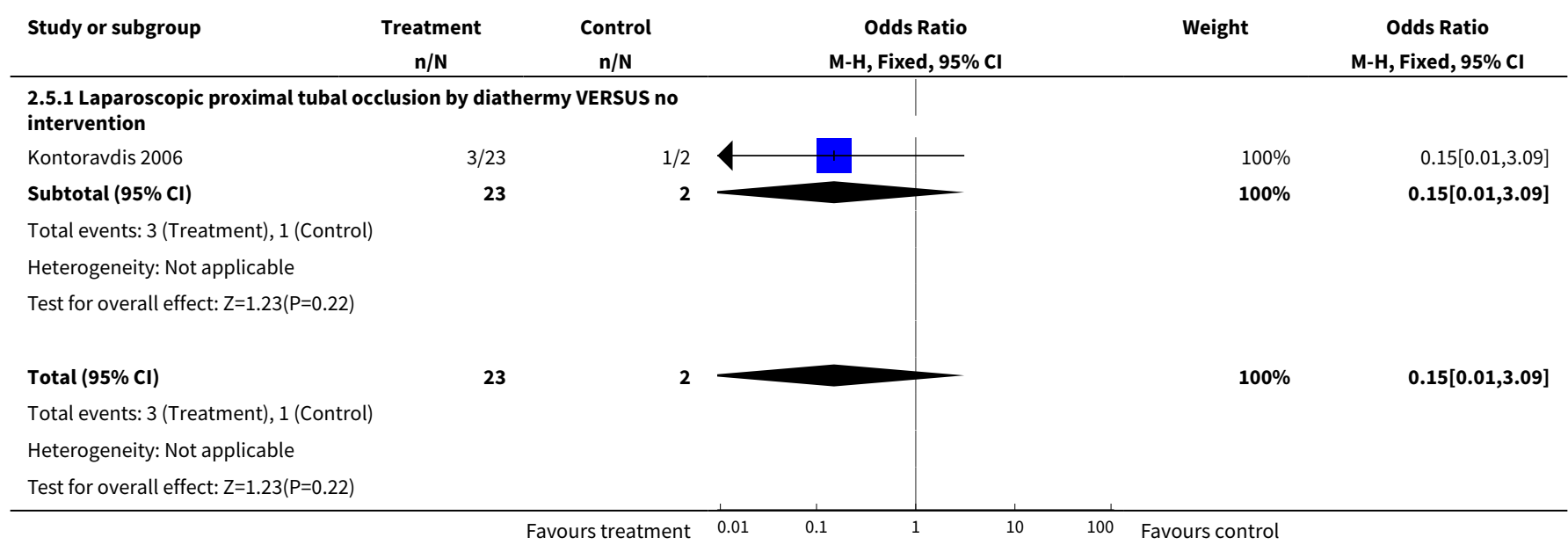

\section{Comparison 3. Aspiration of the hydrosalpinges versus non aspiration of hydrosalpinges}

\begin{tabular}{|c|c|c|c|c|}
\hline Outcome or subgroup title & $\begin{array}{l}\text { No. of } \\
\text { studies }\end{array}$ & $\begin{array}{l}\text { No. of } \\
\text { partici- } \\
\text { pants }\end{array}$ & Statistical method & Effect size \\
\hline 1 Clinical pregnancy rate & 1 & 64 & Odds Ratio (M-H, Fixed, 95\% Cl) & $1.97[0.62,6.29]$ \\
\hline $\begin{array}{l}1.1 \text { Ultrasound guided aspiration of hydrosalp- } \\
\text { inges just after oocyte retrieval VERSUS no aspira- } \\
\text { tion }\end{array}$ & 1 & 64 & Odds Ratio (M-H, Fixed, 95\% Cl) & $1.97[0.62,6.29]$ \\
\hline 2 Biochemical pregnancy rate & 1 & 64 & Odds Ratio (M-H, Fixed, 95\% Cl) & $2.78[0.93,8.27]$ \\
\hline $\begin{array}{l}2.1 \text { Ultrasound guided aspiration of hydrosalp- } \\
\text { inges just after oocyte retrieval VERSUS no aspira- } \\
\text { tion }\end{array}$ & 1 & 64 & Odds Ratio (M-H, Fixed, 95\% Cl) & $2.78[0.93,8.27]$ \\
\hline 3 Pregnancy rate - any definition & 1 & 64 & Odds Ratio (M-H, Fixed, 95\% Cl) & $1.97[0.62,6.29]$ \\
\hline
\end{tabular}




\begin{tabular}{|c|c|c|c|c|}
\hline Outcome or subgroup title & $\begin{array}{l}\text { No. of } \\
\text { studies }\end{array}$ & $\begin{array}{l}\text { No. of } \\
\text { partici- } \\
\text { pants }\end{array}$ & Statistical method & Effect size \\
\hline $\begin{array}{l}3.1 \text { Ultrasound guided aspiration of hydrosalp- } \\
\text { inges just after oocyte retrieval VERSUS no aspira- } \\
\text { tion }\end{array}$ & 1 & 64 & Odds Ratio (M-H, Fixed, 95\% Cl) & $1.97[0.62,6.29]$ \\
\hline 4 Ectopic pregnancy rate & 1 & 66 & Odds Ratio (M-H, Fixed, 95\% Cl) & $0.0[0.0,0.0]$ \\
\hline $\begin{array}{l}4.1 \text { Ultrasound guided aspiration of hydrosalp- } \\
\text { inges just after oocyte retrieval VERSUS no aspira- } \\
\text { tion }\end{array}$ & 1 & 66 & Odds Ratio (M-H, Fixed, 95\% Cl) & $0.0[0.0,0.0]$ \\
\hline 5 Miscarriage rate & 1 & 16 & Odds Ratio (M-H, Fixed, 95\% Cl) & $\begin{array}{l}1.25[0.09 \\
17.65]\end{array}$ \\
\hline $\begin{array}{l}5.1 \text { Ultrasound guided aspiration of hydrosalp- } \\
\text { inges just after oocyte retrieval VERSUS no aspira- } \\
\text { tion }\end{array}$ & 1 & 16 & Odds Ratio (M-H, Fixed, 95\% CI) & $\begin{array}{l}1.25[0.09 \\
17.65]\end{array}$ \\
\hline 6 Surgical complication rate & 1 & 66 & Odds Ratio (M-H, Fixed, 95\% Cl) & $0.0[0.0,0.0]$ \\
\hline $\begin{array}{l}6.1 \text { Ultrasound guided aspiration of hydrosalp- } \\
\text { inges just after oocyte retrieval VERSUS no aspira- } \\
\text { tion }\end{array}$ & 1 & 66 & Odds Ratio (M-H, Fixed, 95\% Cl) & $0.0[0.0,0.0]$ \\
\hline
\end{tabular}

Analysis 3.1. Comparison 3 Aspiration of the hydrosalpinges versus non aspiration of hydrosalpinges, Outcome 1 Clinical pregnancy rate.

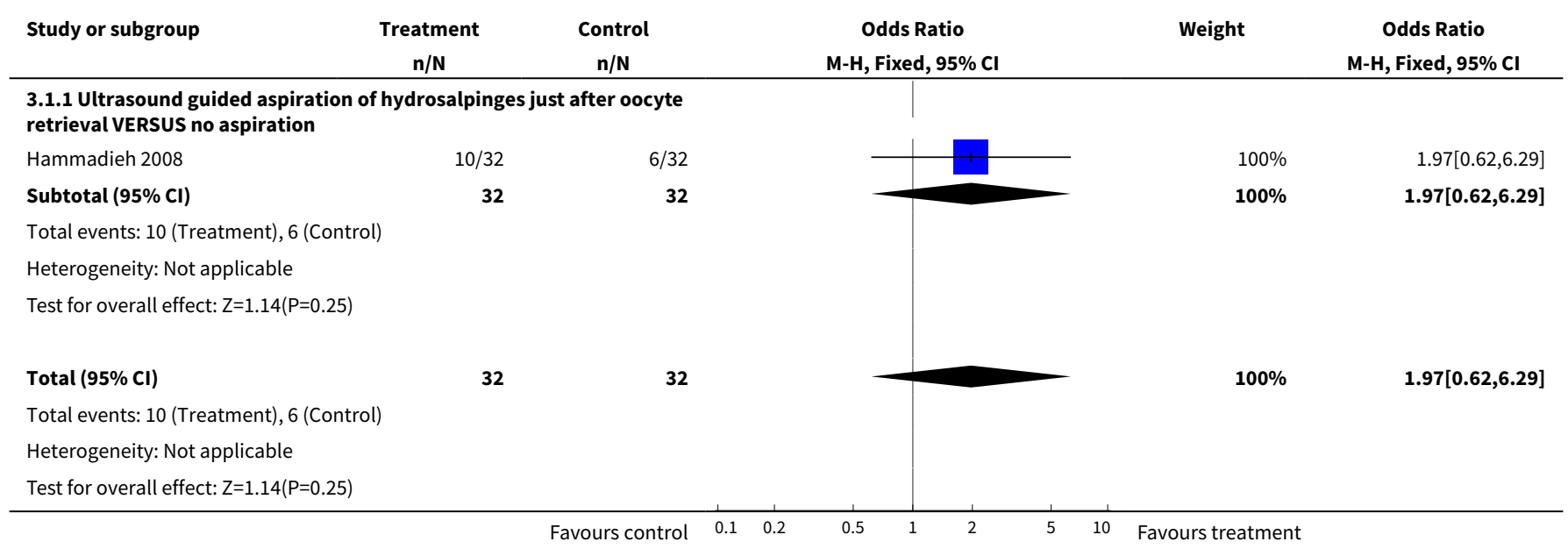


Analysis 3.2. Comparison 3 Aspiration of the hydrosalpinges versus non aspiration of hydrosalpinges, Outcome 2 Biochemical pregnancy rate.

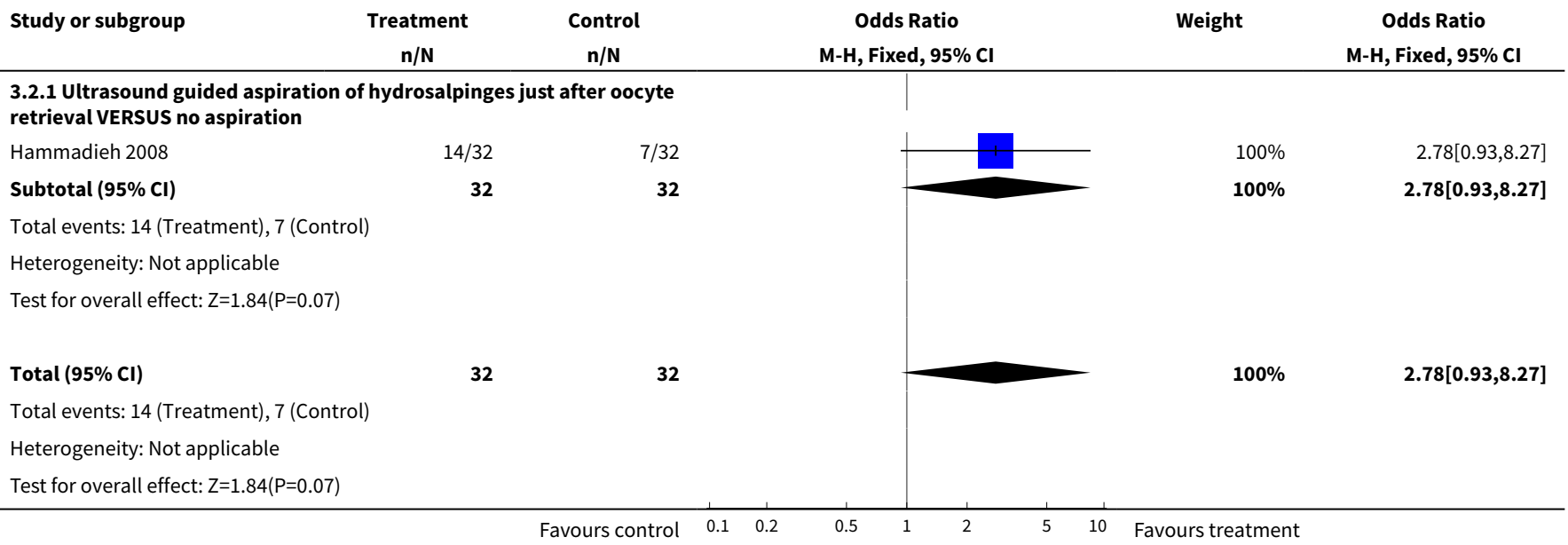

Analysis 3.3. Comparison 3 Aspiration of the hydrosalpinges versus non aspiration of hydrosalpinges, Outcome 3 Pregnancy rate - any definition.

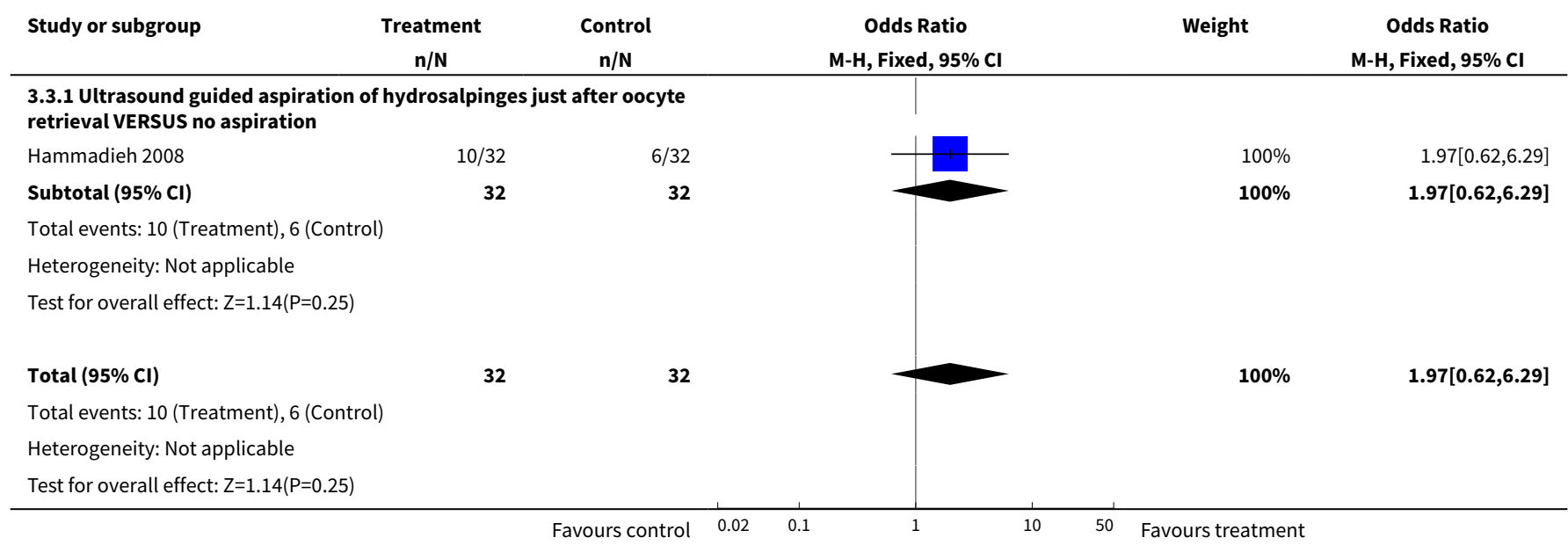

Analysis 3.4. Comparison 3 Aspiration of the hydrosalpinges versus non aspiration of hydrosalpinges, Outcome 4 Ectopic pregnancy rate.

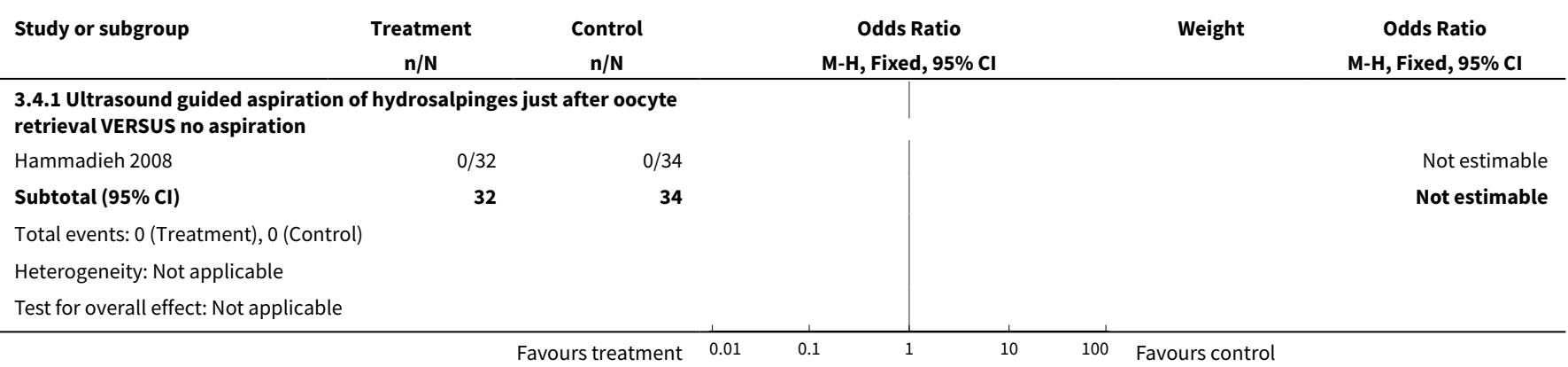




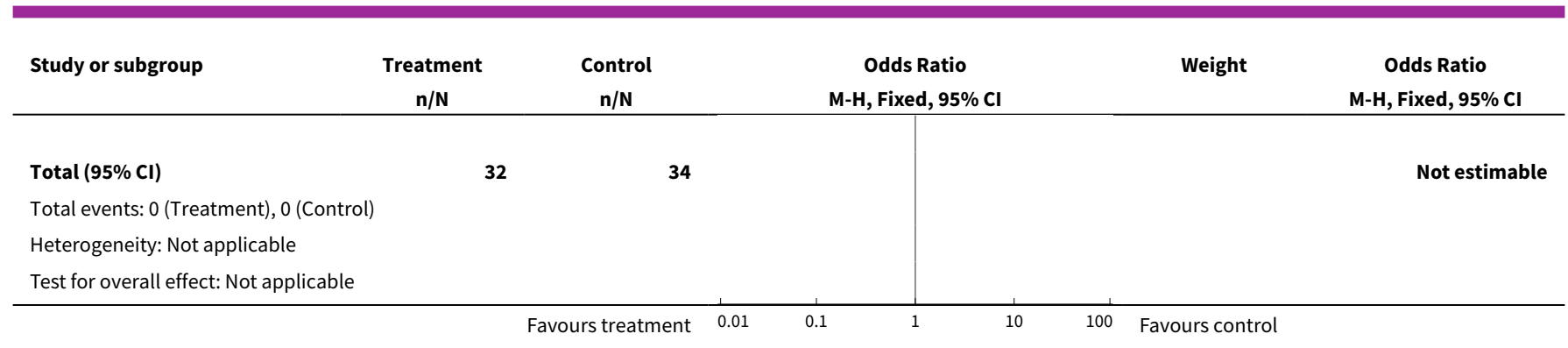

\section{Analysis 3.5. Comparison 3 Aspiration of the hydrosalpinges versus} non aspiration of hydrosalpinges, Outcome 5 Miscarriage rate.

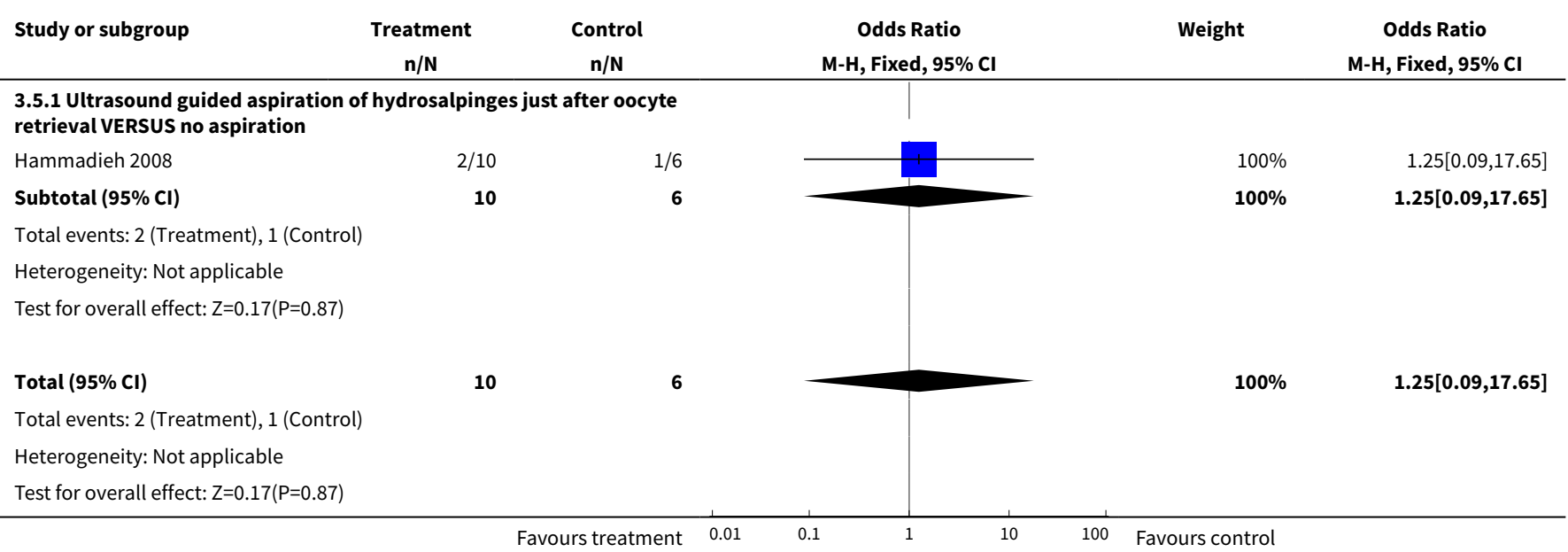

\section{Analysis 3.6. Comparison 3 Aspiration of the hydrosalpinges versus non aspiration of hydrosalpinges, Outcome 6 Surgical complication rate.}

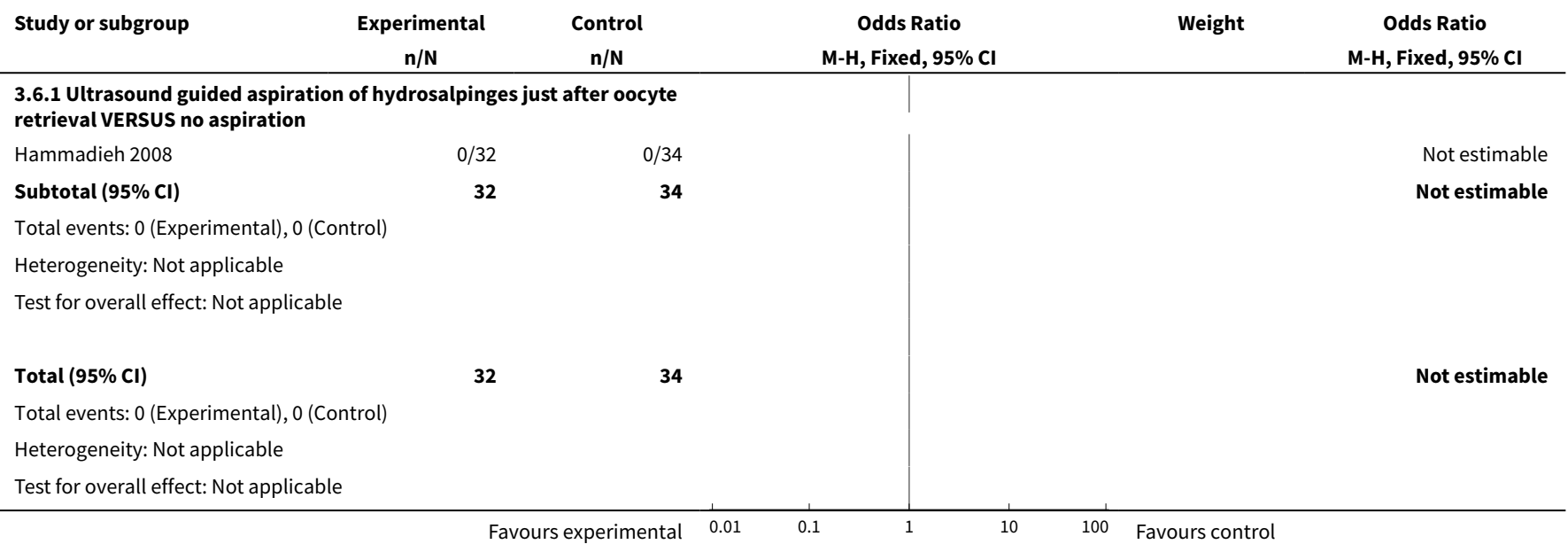


Comparison 4. Laparoscopic surgery on the fallopian tube (all types) VERSUS (any other) laparoscopic surgery on the fallopian tube

\begin{tabular}{|c|c|c|c|c|}
\hline Outcome or subgroup title & $\begin{array}{l}\text { No. of } \\
\text { studies }\end{array}$ & $\begin{array}{l}\text { No. of } \\
\text { partici- } \\
\text { pants }\end{array}$ & Statistical method & Effect size \\
\hline 1 Ongoing pregnancy rate & 1 & 100 & Odds Ratio (M-H, Fixed, 95\% Cl) & $1.65[0.74,3.71]$ \\
\hline $\begin{array}{l}\text { 1.1 Laparoscopic proximal tubal occlusion by } \\
\text { diathermy VERSUS laparoscopic salpingectomy }\end{array}$ & 1 & 100 & Odds Ratio (M-H, Fixed, 95\% Cl) & $1.65[0.74,3.71]$ \\
\hline 2 Clinical pregnancy rate & 2 & 238 & Odds Ratio (M-H, Fixed, 95\% Cl) & $1.28[0.76,2.14]$ \\
\hline $\begin{array}{l}\text { 2.1 Laparoscopic proximal tubal occlusion by } \\
\text { diathermy VERSUS laparoscopic salpingectomy }\end{array}$ & 1 & 100 & Odds Ratio (M-H, Fixed, 95\% Cl) & $1.63[0.74,3.59]$ \\
\hline $\begin{array}{l}\text { 2.2 Laparoscopic proximal tubal clamping } \\
\text { VERSUS laparoscopic salpingectomy }\end{array}$ & 1 & 138 & Odds Ratio (M-H, Fixed, 95\% Cl) & $1.06[0.53,2.12]$ \\
\hline 3 Pregnancy rate - any definition & 2 & 238 & Odds Ratio (M-H, Fixed, 95\% Cl) & $1.28[0.76,2.14]$ \\
\hline $\begin{array}{l}\text { 3.1 Laparoscopic proximal tubal occlusion by } \\
\text { diathermy VERSUS laparoscopic salpingectomy }\end{array}$ & 1 & 100 & Odds Ratio (M-H, Fixed, 95\% Cl) & $1.63[0.74,3.59]$ \\
\hline $\begin{array}{l}\text { 3.2 Laparoscopic proximal tubal clamping } \\
\text { VERSUS laparoscopic salpingectomy }\end{array}$ & 1 & 138 & Odds Ratio (M-H, Fixed, 95\% Cl) & $1.06[0.53,2.12]$ \\
\hline 4 Ectopic pregnancy rate & 1 & 100 & Odds Ratio (M-H, Fixed, 95\% Cl) & $3.06[0.12,76.95]$ \\
\hline $\begin{array}{l}\text { 4.1 Laparoscopic proximal tubal occlusion by } \\
\text { diathermy VERSUS laparoscopic salpingectomy }\end{array}$ & 1 & 100 & Odds Ratio (M-H, Fixed, 95\% Cl) & $3.06[0.12,76.95]$ \\
\hline 5 Miscarriage rate & 1 & 43 & Odds Ratio (M-H, Fixed, 95\% Cl) & $1.35[0.20,9.02]$ \\
\hline $\begin{array}{l}5.1 \text { Laparoscopic proximal tubal occlusion by } \\
\text { diathermy VERSUS laparoscopic salpingectomy }\end{array}$ & 1 & 43 & Odds Ratio (M-H, Fixed, 95\% Cl) & $1.35[0.20,9.02]$ \\
\hline
\end{tabular}

Analysis 4.1. Comparison 4 Laparoscopic surgery on the fallopian tube (all types) VERSUS (any other) laparoscopic surgery on the fallopian tube, Outcome 1 Ongoing pregnancy rate.

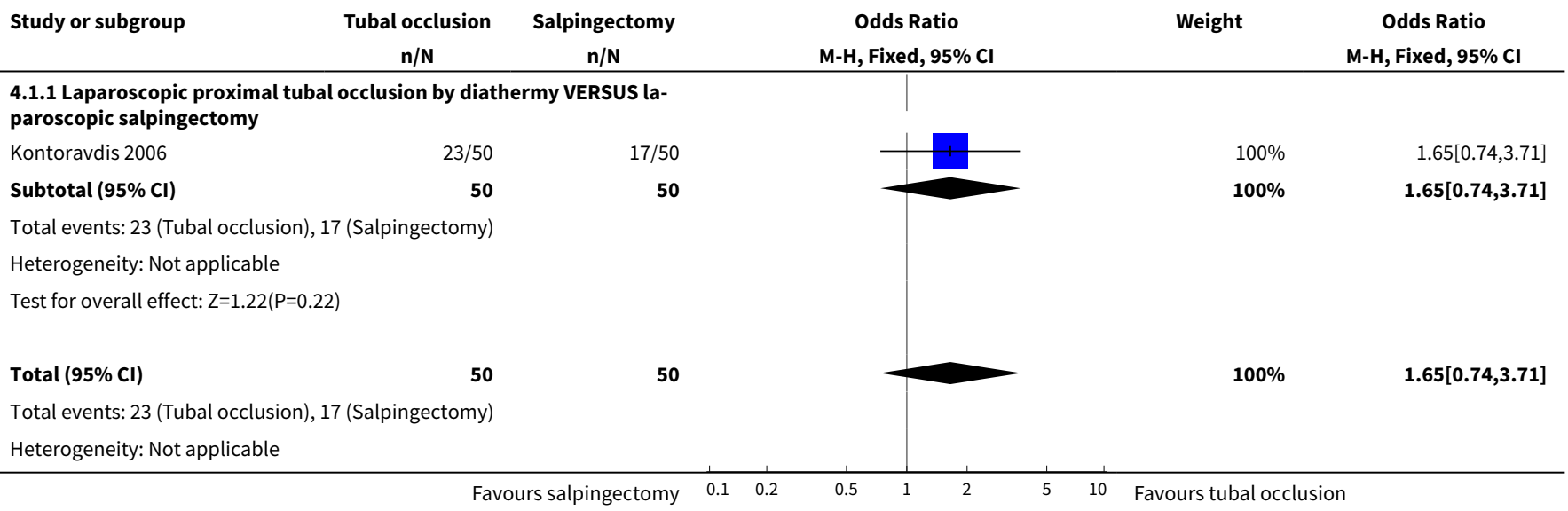




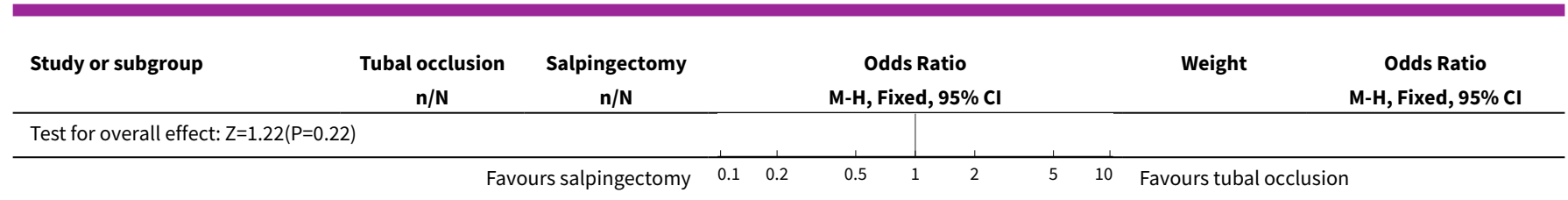

Analysis 4.2. Comparison 4 Laparoscopic surgery on the fallopian tube (all types) VERSUS (any other) laparoscopic surgery on the fallopian tube, Outcome 2 Clinical pregnancy rate.

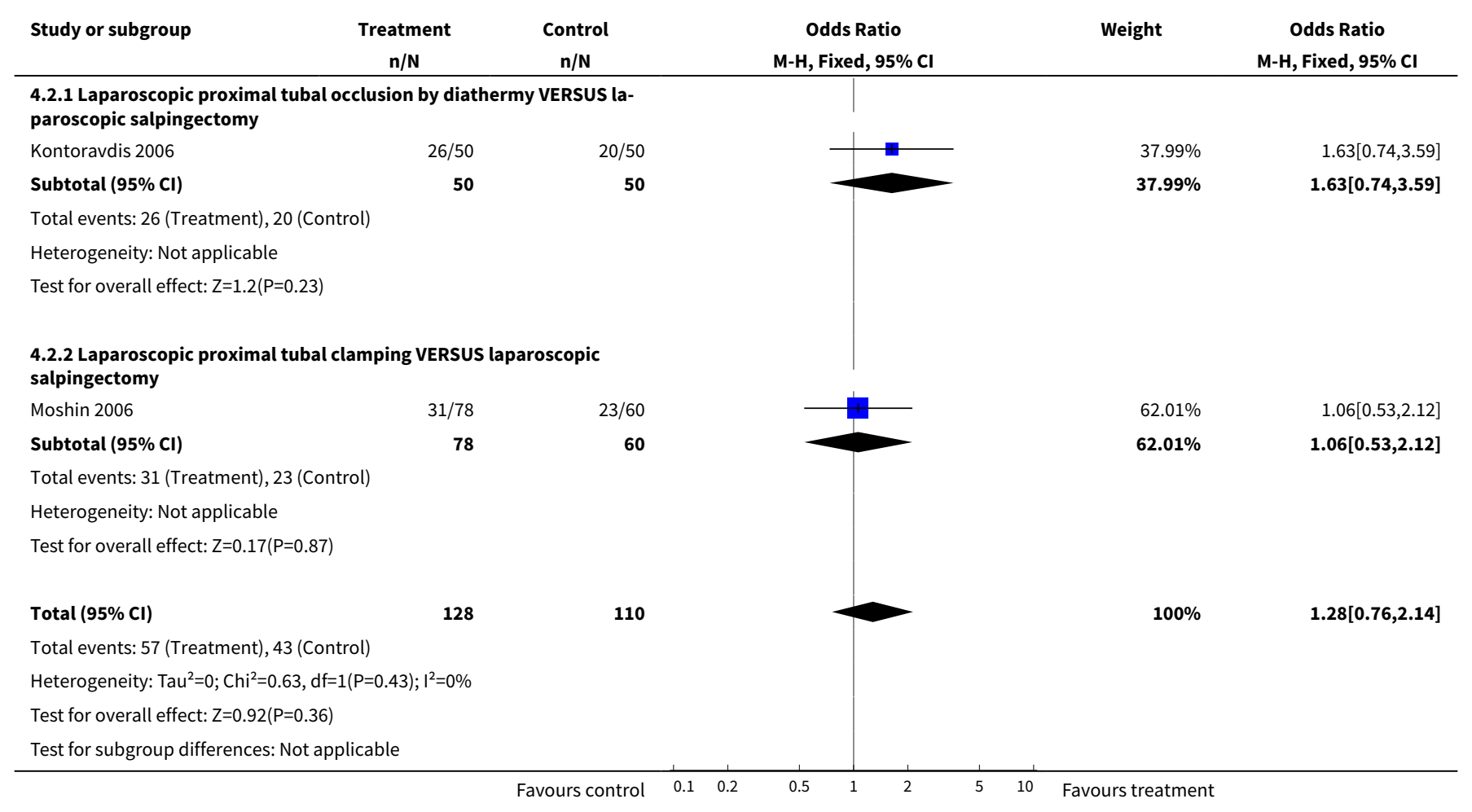

Analysis 4.3. Comparison 4 Laparoscopic surgery on the fallopian tube (all types) VERSUS (any other) laparoscopic surgery on the fallopian tube, Outcome 3 Pregnancy rate - any definition.

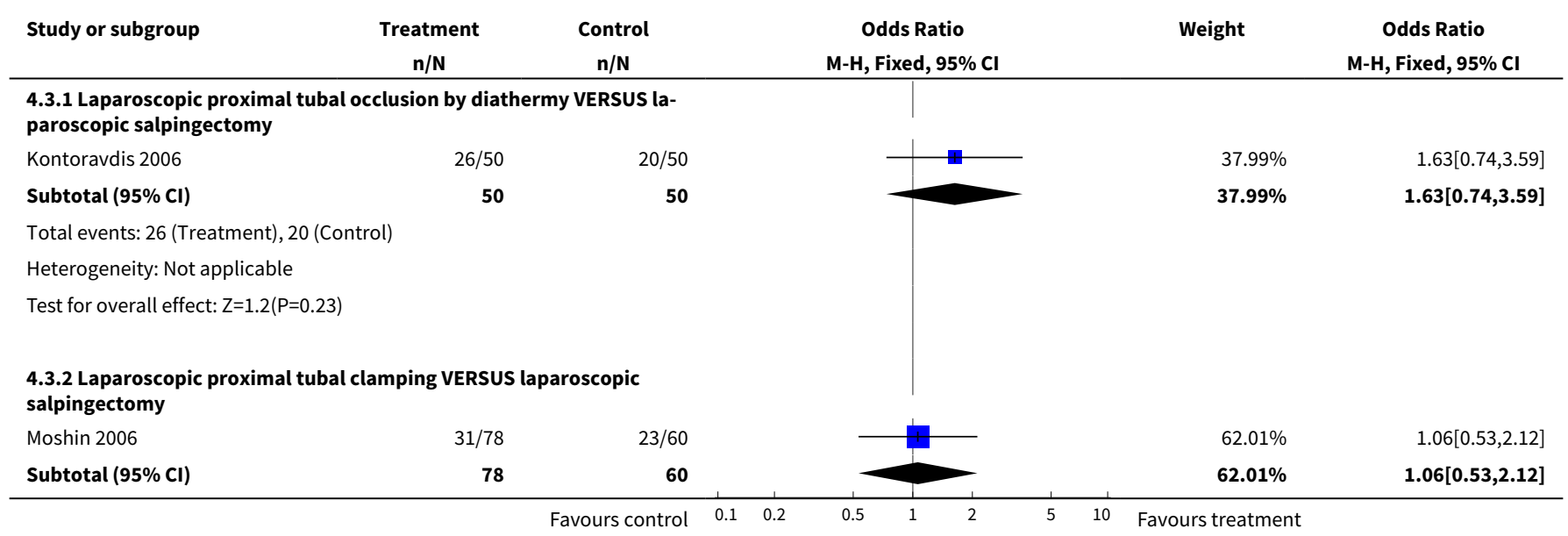




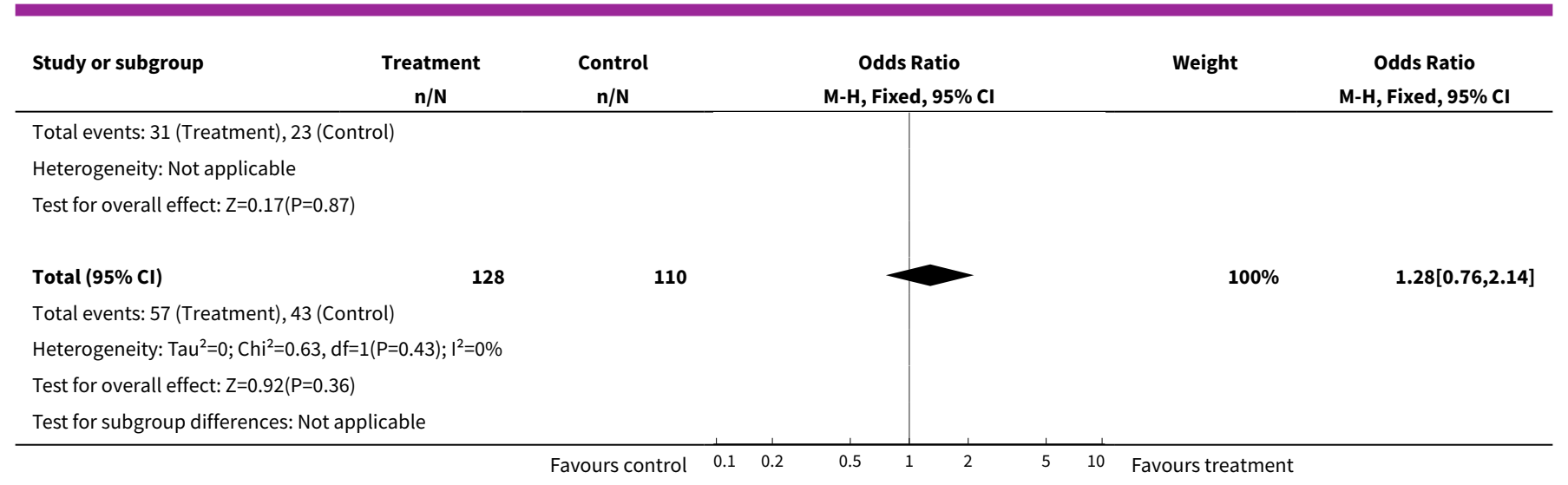

Analysis 4.4. Comparison 4 Laparoscopic surgery on the fallopian tube (all types) VERSUS (any other) laparoscopic surgery on the fallopian tube, Outcome 4 Ectopic pregnancy rate.

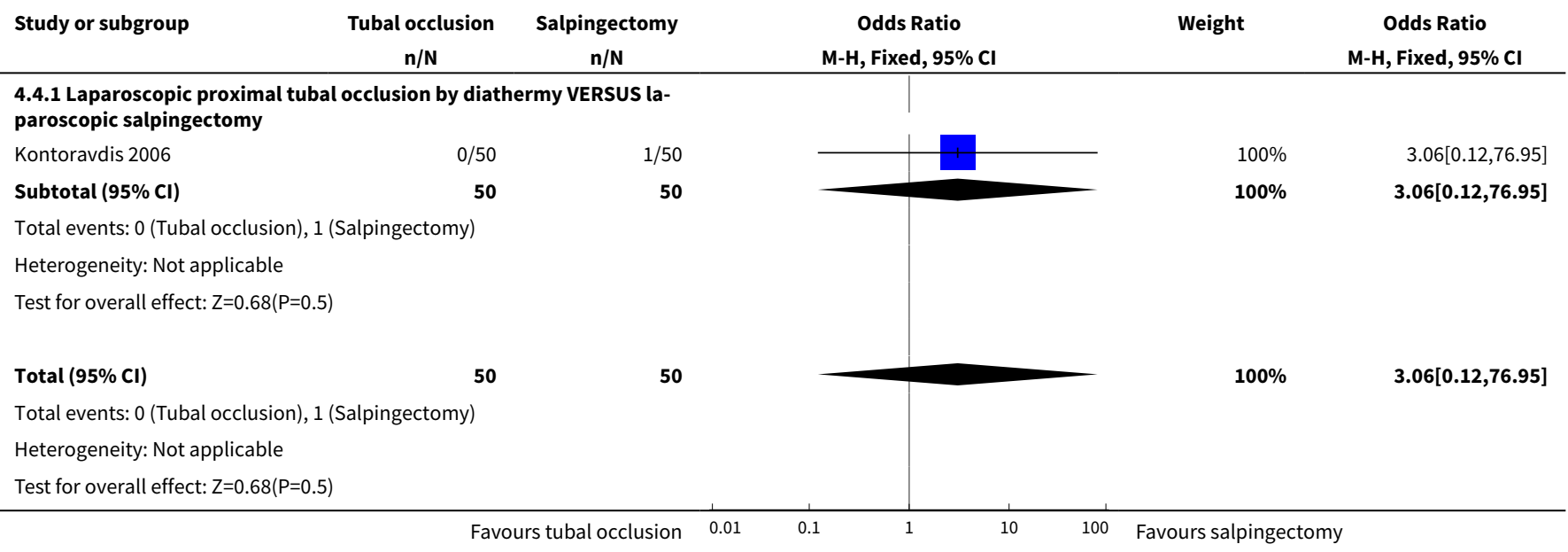

\section{Analysis 4.5. Comparison 4 Laparoscopic surgery on the fallopian tube (all types) VERSUS (any other) laparoscopic surgery on the fallopian tube, Outcome 5 Miscarriage rate.}

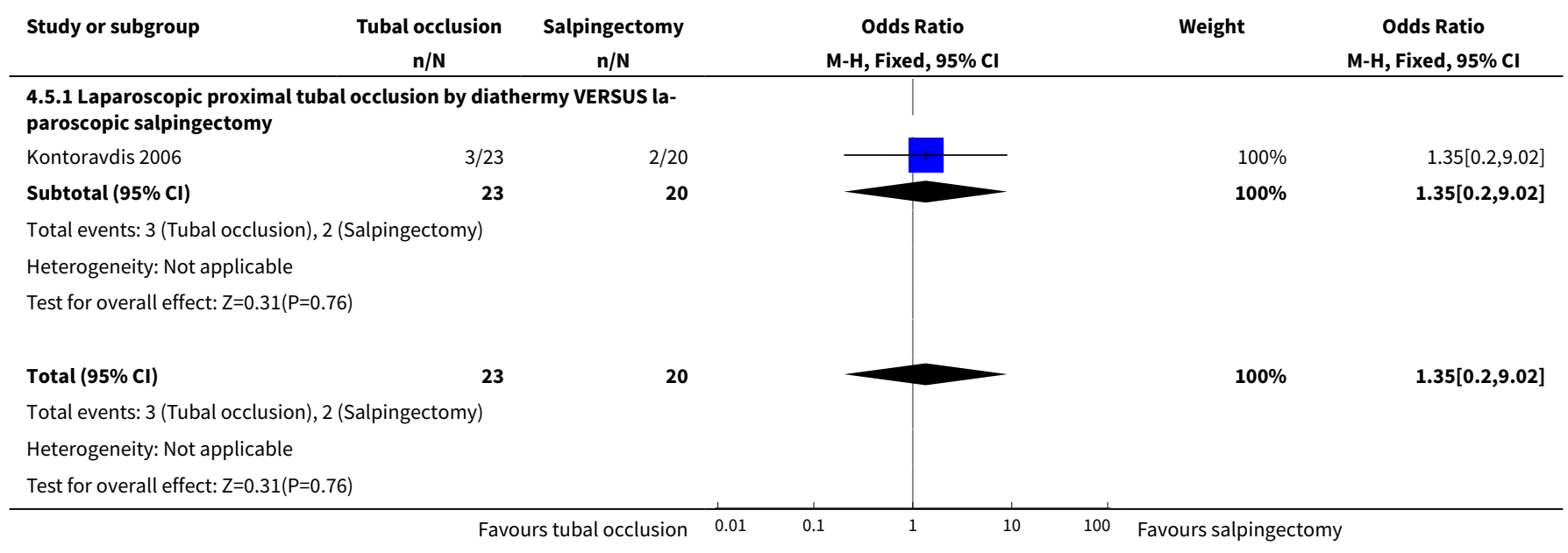


Comparison 5. Surgical treatment (all types) VERSUS no surgical treatment

\begin{tabular}{|c|c|c|c|c|}
\hline Outcome or subgroup title & $\begin{array}{l}\text { No. of } \\
\text { studies }\end{array}$ & $\begin{array}{l}\text { No. of } \\
\text { partici- } \\
\text { pants }\end{array}$ & Statistical method & Effect size \\
\hline $\begin{array}{l}1 \text { Salpingectomy (all methods) } \\
\text { VERSUS no surgical treatment }\end{array}$ & 4 & & Odds Ratio (M-H, Fixed, 95\% Cl) & Subtotals only \\
\hline 1.1 Ongoing pregnancy rate & 3 & 329 & Odds Ratio (M-H, Fixed, 95\% Cl) & $2.20[1.26,3.82]$ \\
\hline 1.2 Clinical pregnancy rate & 3 & 395 & Odds Ratio (M-H, Fixed, 95\% Cl) & $2.40[1.49,3.86]$ \\
\hline $\begin{array}{l}\text { 1.3 Pregnancy rate - any defin- } \\
\text { ition }\end{array}$ & 4 & 455 & Odds Ratio (M-H, Fixed, 95\% Cl) & $2.49[1.60,3.86]$ \\
\hline 1.4 Ectopic pregnancy rate & 3 & 329 & Odds Ratio (M-H, Fixed, 95\% Cl) & $0.64[0.15,2.75]$ \\
\hline 1.5 Miscarriage rate & 3 & 329 & Odds Ratio (M-H, Fixed, 95\% Cl) & $0.86[0.31,2.38]$ \\
\hline 1.6 Surgical complication rate & 1 & 204 & Odds Ratio (M-H, Fixed, 95\% Cl) & $3.86[0.18,81.52]$ \\
\hline $\begin{array}{l}2 \text { Tubal occlusion (all meth- } \\
\text { ods) VERSUS no surgical treat- } \\
\text { ment }\end{array}$ & 2 & & Odds Ratio (M-H, Fixed, 95\% Cl) & Subtotals only \\
\hline 2.1 Ongoing pregnancy rate & 1 & 65 & Odds Ratio (M-H, Fixed, 95\% Cl) & $7.21[0.87,59.57]$ \\
\hline 2.2 Clinical pregnancy rate & 2 & 209 & Odds Ratio (M-H, Fixed, 95\% Cl) & $4.66[2.17,10.01]$ \\
\hline 2.3 Ectopic pregnancy rate & 1 & 65 & Odds Ratio (M-H, Fixed, 95\% Cl) & $0.94[0.04,24.25]$ \\
\hline 2.4 Miscarriage rate & 1 & 65 & Odds Ratio (M-H, Fixed, 95\% Cl) & $0.89[0.09,9.28]$ \\
\hline $\begin{array}{l}3 \text { Aspiration of hydro salp- } \\
\text { ingeal fluid (all methods) } \\
\text { VERSUS no surgical treatment }\end{array}$ & 1 & & Odds Ratio (M-H, Fixed, 95\% Cl) & Subtotals only \\
\hline 3.1 Clinical pregnancy rate & 1 & 64 & Odds Ratio (M-H, Fixed, 95\% Cl) & $1.97[0.62,6.29]$ \\
\hline $\begin{array}{l}3.2 \text { Biochemical pregnancy } \\
\text { rate }\end{array}$ & 1 & 64 & Odds Ratio (M-H, Fixed, 95\% Cl) & $2.78[0.93,8.27]$ \\
\hline 3.3 Ectopic pregnancy rate & 1 & 64 & Odds Ratio (M-H, Fixed, 95\% Cl) & $0.0[0.0,0.0]$ \\
\hline 3.4 Miscarriage rate & 1 & 64 & Odds Ratio (M-H, Fixed, 95\% Cl) & $2.07[0.18,24.01]$ \\
\hline 3.5 Surgical complication rate & 1 & 64 & Odds Ratio (M-H, Fixed, 95\% Cl) & $0.0[0.0,0.0]$ \\
\hline
\end{tabular}


Analysis 5.1. Comparison 5 Surgical treatment (all types) VERSUS no surgical treatment, Outcome 1 Salpingectomy (all methods) VERSUS no surgical treatment.

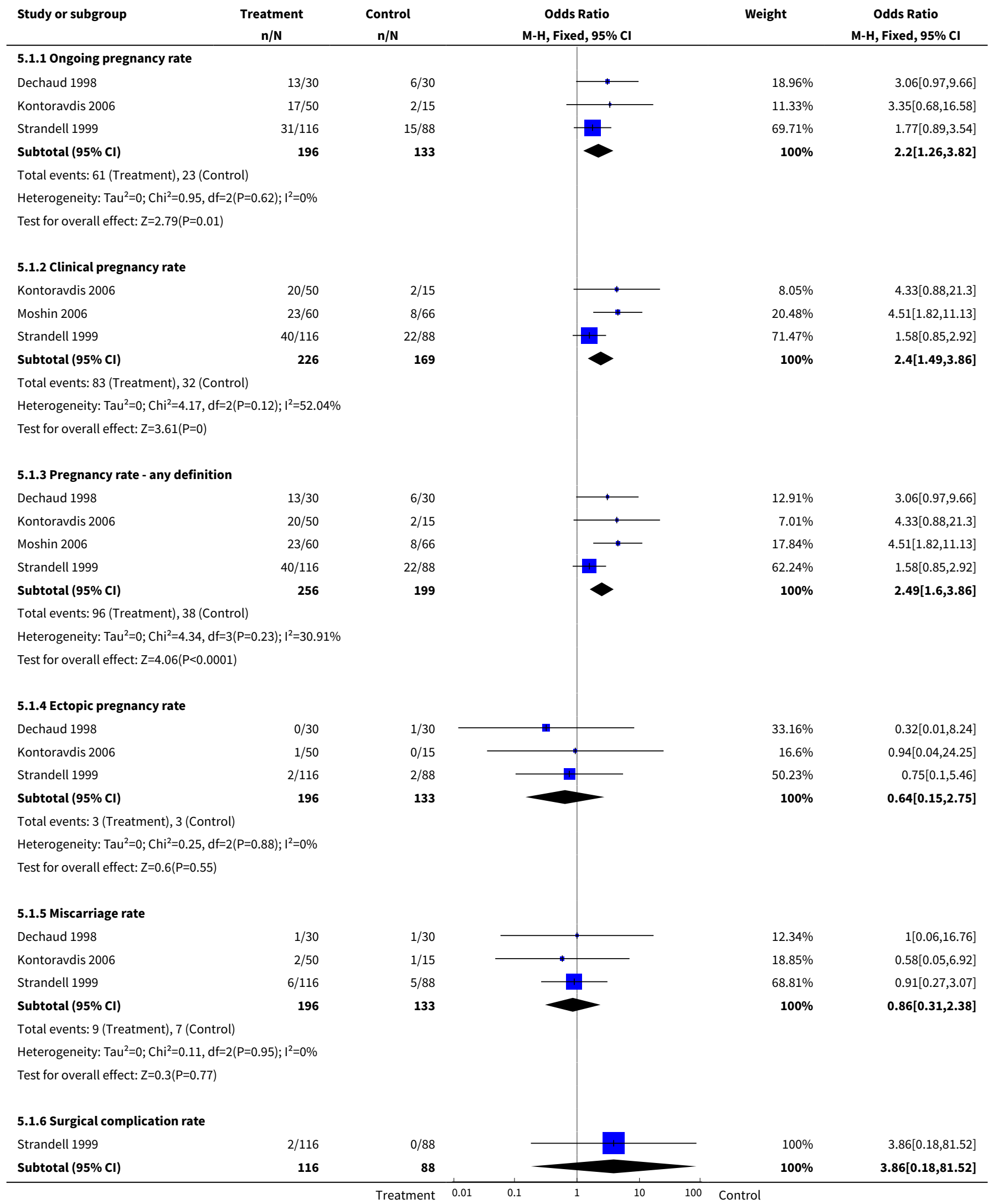




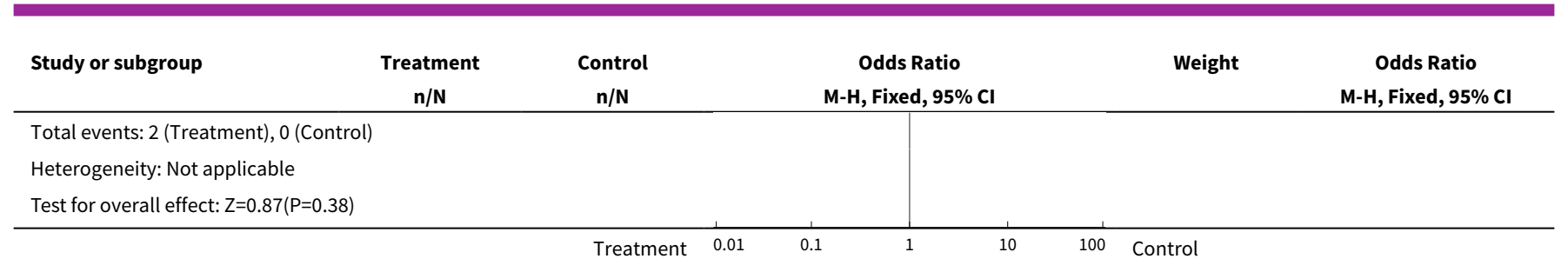

Analysis 5.2. Comparison 5 Surgical treatment (all types) VERSUS no surgical treatment, Outcome 2 Tubal occlusion (all methods) VERSUS no surgical treatment.

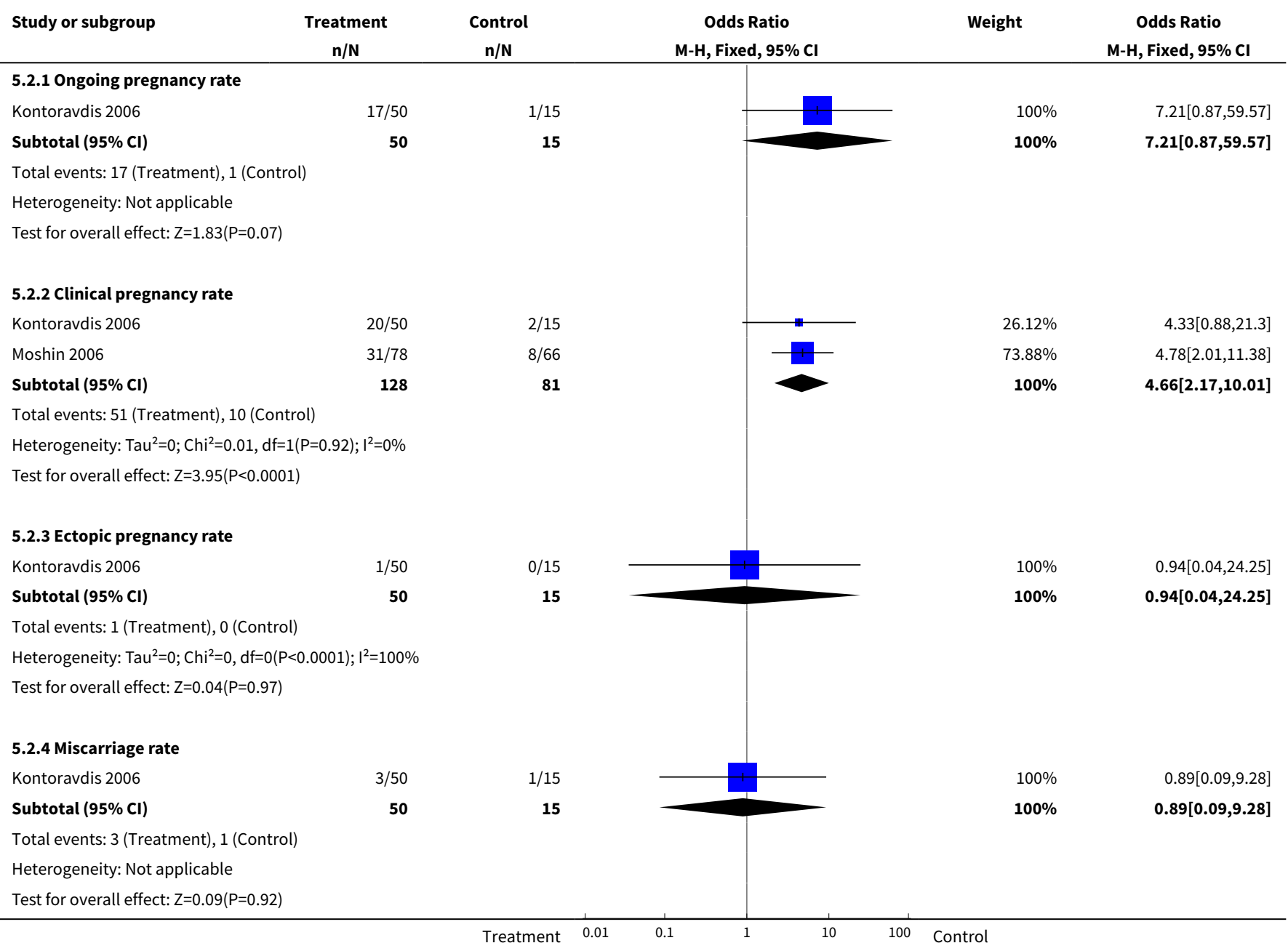

Analysis 5.3. Comparison 5 Surgical treatment (all types) VERSUS no surgical treatment, Outcome 3 Aspiration of hydro salpingeal fluid (all methods) VERSUS no surgical treatment.

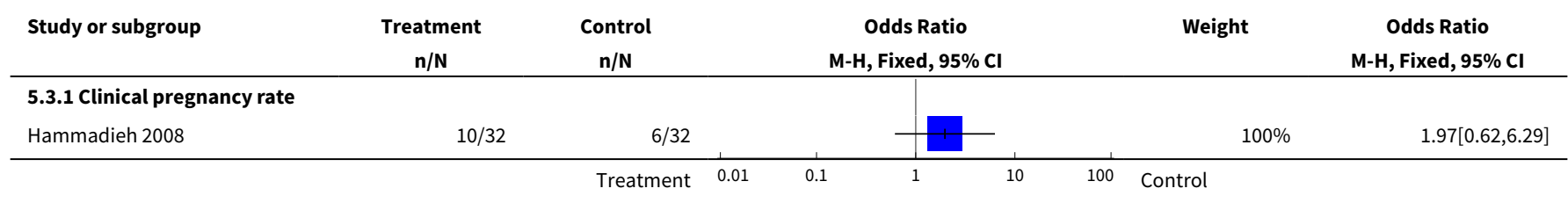




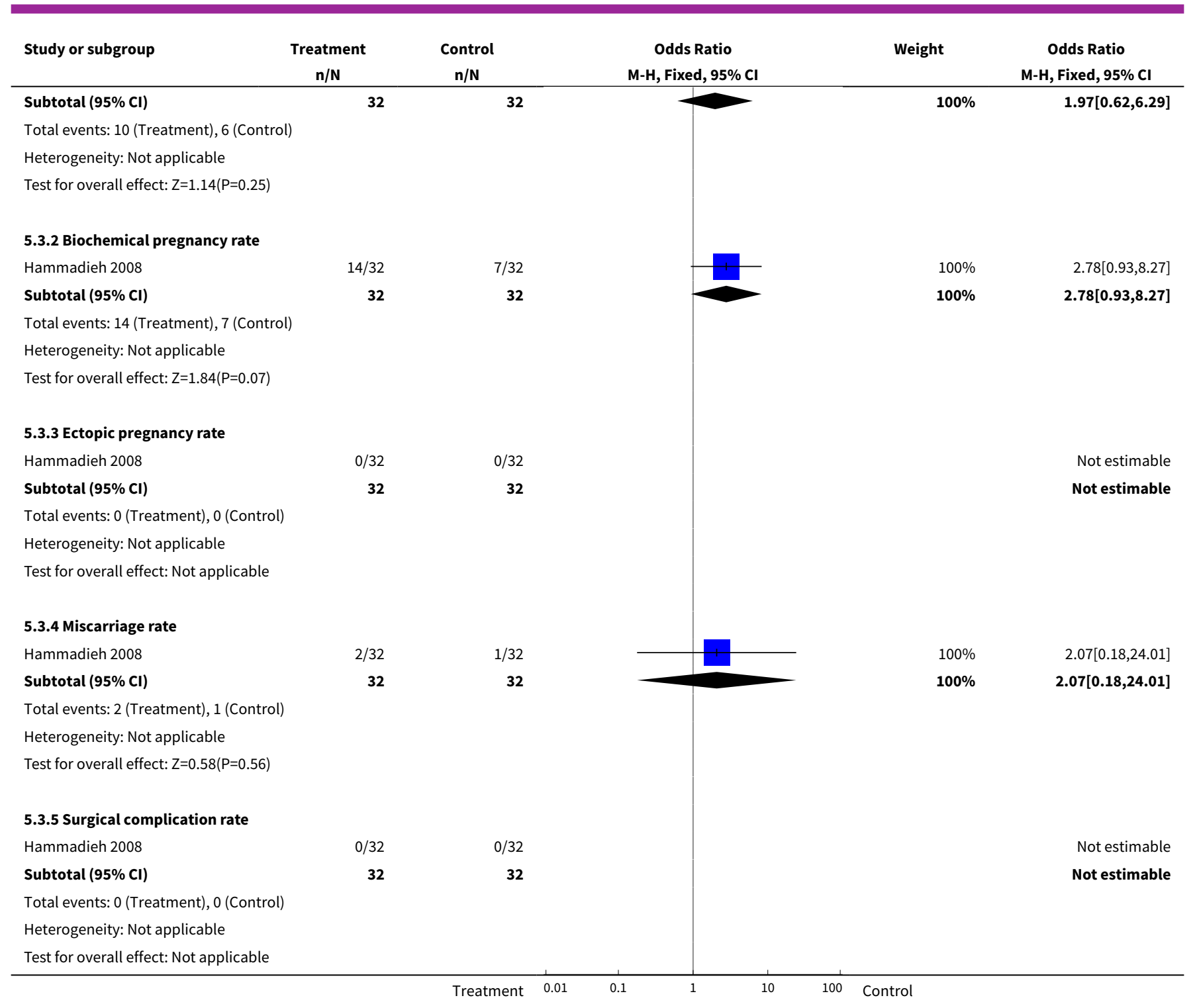

Comparison 6. Laparoscopic surgery on the fallopian tube (all types) VERSUS (any other) laparoscopic surgery on the fallopian tube

\begin{tabular}{lllll}
\hline Outcome or subgroup title & $\begin{array}{l}\text { No. of } \\
\text { studies }\end{array}$ & $\begin{array}{l}\text { No. of } \\
\text { partici- } \\
\text { pants }\end{array}$ & Statistical method & Effect size \\
\hline $\begin{array}{l}1 \text { Tubal occlusion (all methods) } \\
\text { VERSUS Salpingectomy (all meth- } \\
\text { ods) }\end{array}$ & 2 & & Odds Ratio (M-H, Fixed, 95\% Cl) & Subtotals only \\
\hline $\begin{array}{lllll}1.1 \text { Ongoing pregnancy rate } \\
1.2 \text { Clinical pregnancy rate }\end{array}$ & 1 & 100 & Odds Ratio (M-H, Fixed, 95\% Cl) & $1.65[0.74,3.71]$ \\
\hline 1.3 Ectopic pregnancy rate & 1 & 238 & Odds Ratio (M-H, Fixed, 95\% Cl) & $1.28[0.76,2.14]$ \\
\hline
\end{tabular}




\begin{tabular}{lllll}
\hline Outcome or subgroup title & $\begin{array}{l}\text { No. of } \\
\text { studies }\end{array}$ & $\begin{array}{l}\text { No. of } \\
\text { partici- } \\
\text { pants }\end{array}$ & Statistical method & Effect size \\
\hline 1.4 Miscarriage rate & 1 & 100 & Odds Ratio (M-H, Fixed, 95\% Cl) & $1.53[0.24,9.59]$ \\
\hline
\end{tabular}

Analysis 6.1. Comparison 6 Laparoscopic surgery on the fallopian tube (all types) VERSUS (any other) laparoscopic surgery on the fallopian tube, Outcome 1 Tubal occlusion (all methods) VERSUS Salpingectomy (all methods).

\begin{tabular}{|c|c|c|c|c|c|}
\hline Study or subgroup & $\begin{array}{c}\text { Tubal occlusion } \\
n / N\end{array}$ & $\begin{array}{c}\text { Salpingectomy } \\
n / N\end{array}$ & $\begin{array}{c}\text { Odds Ratio } \\
\text { M-H, Fixed, } 95 \% \mathrm{Cl}\end{array}$ & Weight & $\begin{array}{c}\text { Odds Ratio } \\
\text { M-H, Fixed, } 95 \% \mathrm{Cl}\end{array}$ \\
\hline \multicolumn{6}{|c|}{ 6.1.1 Ongoing pregnancy rate } \\
\hline Kontoravdis 2006 & $23 / 50$ & $17 / 50$ & & $100 \%$ & $1.65[0.74,3.71]$ \\
\hline Subtotal $(95 \% \mathrm{Cl})$ & 50 & 50 & & $100 \%$ & $1.65[0.74,3.71]$ \\
\hline \multicolumn{6}{|c|}{ Total events: 23 (Tubal occlusion), 17 (Salpingectomy) } \\
\hline \multicolumn{6}{|c|}{ Heterogeneity: Not applicable } \\
\hline \multicolumn{6}{|c|}{ Test for overall effect: $Z=1.22(P=0.22)$} \\
\hline \multicolumn{6}{|c|}{ 6.1.2 Clinical pregnancy rate } \\
\hline Kontoravdis 2006 & $26 / 50$ & $20 / 50$ & 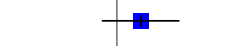 & $37.99 \%$ & $1.63[0.74,3.59]$ \\
\hline Moshin 2006 & $31 / 78$ & $23 / 60$ & & $62.01 \%$ & $1.06[0.53,2.12]$ \\
\hline Subtotal $(95 \% \mathrm{Cl})$ & 128 & 110 & & $100 \%$ & $1.28[0.76,2.14]$ \\
\hline \multicolumn{6}{|c|}{ Total events: 57 (Tubal occlusion), 43 (Salpingectomy) } \\
\hline \multicolumn{6}{|c|}{ Heterogeneity: Tau $^{2}=0 ; \mathrm{Chi}^{2}=0.63, \mathrm{df}=1(\mathrm{P}=0.43) ;\left.\right|^{2}=0 \%$} \\
\hline \multicolumn{6}{|c|}{ Test for overall effect: $\mathrm{Z}=0.92(\mathrm{P}=0.36)$} \\
\hline \multicolumn{6}{|c|}{ 6.1.3 Ectopic pregnancy rate } \\
\hline Kontoravdis 2006 & $0 / 50$ & $1 / 50$ & & $100 \%$ & $0.33[0.01,8.21]$ \\
\hline Subtotal $(95 \% \mathrm{Cl})$ & 50 & 50 & & $100 \%$ & $0.33[0.01,8.21]$ \\
\hline \multicolumn{6}{|c|}{ Total events: 0 (Tubal occlusion), 1 (Salpingectomy) } \\
\hline \multicolumn{6}{|c|}{ Heterogeneity: Not applicable } \\
\hline \multicolumn{6}{|c|}{ Test for overall effect: $\mathrm{Z}=0.68(\mathrm{P}=0.5)$} \\
\hline \multicolumn{6}{|c|}{ 6.1.4 Miscarriage rate } \\
\hline Kontoravdis 2006 & $3 / 50$ & $2 / 50$ & & $100 \%$ & $1.53[0.24,9.59]$ \\
\hline Subtotal $(95 \% \mathrm{Cl})$ & 50 & 50 & & $100 \%$ & $1.53[0.24,9.59]$ \\
\hline \multicolumn{6}{|c|}{ Total events: 3 (Tubal occlusion), 2 (Salpingectomy) } \\
\hline \multicolumn{6}{|c|}{ Heterogeneity: Not applicable } \\
\hline Test for overall effec & & & & & \\
\hline
\end{tabular}

\section{APPENDICES}

\section{Appendix 1. Search strategy}

\section{Database: Cochrane Menstrual Disorders and Subfertility Group (MDSG) trials register}

Keywords CONTAINS "IVF" or "ICSI" or "in-vitro fertilisation " or "in-vitro fertilisation procedure" or "in-vitro fertilisation procedure failure" or "in-vitro fertilisation techniques" or "in vitro fertilization" or "intracytoplasmic sperm injection" or "intracytoplasmic sperm injection techniques" or Title CONTAINS "IVF" or "ICSI" or "in-vitro fertilisation " or "in-vitro fertilisation procedure" or "in-vitro fertilisation 
procedure failure" or "in-vitro fertilisation techniques" or "in vitro fertilization" or "intracytoplasmic sperm injection" or "intracytoplasmic sperm injection techniques"

AND

Keywords CONTAINS "tubal anastomosis"or "tubal disorders" or "tubal factor" or "tubal occlusion" or "tubal patency" or "tubal reconstruction" or "tubal subfertility" or "tuboplasty" or "tube drainage" or "Fallopian tube obstruction"or "Fallopian Tube Fixation" or "falloscopy" or "salpingectomy" or "Salpingolysis" or "^Salpingostomy-" or"salpingotomy" or "hydrosalpingies" or "Hydrosalpinx" or "hydrosalpinges" or "hydrotubation" or "Aspiration" or Title CONTAINS "tubal anastomosis"or "tubal disorders" or "tubal factor" or "tubal occlusion" or "tubal patency" or "tubal reconstruction" or "tubal subfertility" or "tuboplasty" or "tube drainage" or "Fallopian tube obstruction"or "Fallopian Tube Fixation" or "falloscopy" or "salpingectomy" or "Salpingolysis" or "*Salpingostomy-" or"salpingotomy" or "hydrosalpingies" or "Hydrosalpinx" or "hydrosalpinges" or "hydrotubation" or "Aspiration"

\section{Database: Cochrane Central Register of Controlled Trials}

1 (in vitro fertilisation or in vitro fertilization).tw. (1148)

2 (ivf or icsi).tw. (1793)

3 intracytoplasmic sperm injections.tw. (0)

4 exp reproductive techniques, assisted/or exp embryo transfer/ or exp fertilization in vitro/ or exp sperm injections, intracytoplasmic/ or exp zygote intrafallopian transfer/ (1740)

5 (ART or embryo transfer or et).tw. (4591)

6 or/1-5 (6699)

7 exp Fallopian Tube Diseases/ (94)

8 Fallopian Tube Disease\$.tw. (0)

9 salping\$.tw. (204)

10 (fallopian adj3 disease\$).tw. (0)

11 (tub\$ adj5 adhesion\$).tw. (33)

12 (tub\$ adj3 occlusion\$).tw. (94)

13 (tub\$ adj5 block\$).tw. (121)

14 hydrosalpin\$.tw. (29)

15 (surg\$ adj5 tub\$).tw. (307)

16 (aspirat\$ adj5 hydrosalpin\$).tw. (2)

17 essure\$.tw. (1)

18 (clip\$ adj5 hydrosalpin\$).tw. (1)

19 hysteroscop\$.tw. (326)

20 or/7-19 (1063)

216 and 20 (67)

22 limit 21 to $y r=" 2004-2008$ " (15)

23 from 22 keep 1-15 (15)

\section{Database: Ovid MEDLINE(R)}

1 (in vitro fertilisation or in vitro fertilization).tw. (12679)

2 (ivf or icsi).tw. (13008)

3 intracytoplasmic sperm injections.tw. (15)

4 exp reproductive techniques, assisted/ or exp embryo transfer/ or exp fertilization in vitro/ or exp sperm injections, intracytoplasmic/ or exp zygote intrafallopian transfer/ (41350)

5 (ART or embryo transfer or et).tw. (137241)

6 or/1-5 (174789)

7 exp Fallopian Tube Diseases/ (5463)

8 Fallopian Tube Disease\$.tw. (11)

9 salping\$.tw. (5123)

10 (fallopian adj3 disease\$).tw. (37)

11 (tub\$ adj5 adhesion\$).tw. (669)

12 (tub\$ adj3 occlusion\$).tw. (967)

13 (tub\$ adj5 block\$).tw. (2032)

14 hydrosalpin\$.tw. (532)

15 (surg\$ adj5 tub\$).tw. (6164)

16 (aspirat\$ adj5 hydrosalpin\$).tw. (13)

17 essure\$.tw. (56)

18 (clip\$ adj5 hydrosalpin\$).tw. (4)

19 hysteroscop\$.tw. (3330)

20 or/7-19 (21124)

216 and 20 (1119)

22 randomised controlled trial.pt. (263468)

Surgical treatment for tubal disease in women due to undergo in vitro fertilisation (Review)

Copyright (c) 2010 The Cochrane Collaboration. Published by John Wiley \& Sons, Ltd. 
23 controlled clinical trial.pt. (79901)

24 randomized.ab. (172034)

25 placebo.ab. (109112)

26 cross-over studies/ (22777)

27 (crossover or cross-over or cross over).tw. (41720)

28 clinical trials as topic.sh. (142719)

29 randomly.ab. (124831)

30 trial.ti. (76577)

31 or/22-30 (623968)

32 humans.sh. (10628562)

$33 \quad 31$ and 32 (564390)

3433 and 21 (68)

35 limit 34 to $y r=" 2004-2008 "(21)$

36 from 35 keep 1-21 (21)

\section{Database: EMBASE}

1 exp embryo transfer/ or exp fertilization in vitro/ or exp intracytoplasmic sperm injection/ (24650)

2 (in vitro fertilisation or in vitro fertilization).tw. (11070)

3 (ivf or icsi or et or embryo transfer).tw. (143156)

4 intracytoplasmic sperm injection.tw. (3040)

5 or/1-4 (155969)

6 (tub\$ adj3 disease\$).tw. (4190)

7 (fallopian adj5 disease).tw. (42)

8 (tub\$ adj5 adhesion\$).tw. (573)

9 (tub\$ adj5 occlusion\$).tw. (886)

10 (tub\$ adj5 block\$).tw. (1619)

11 (fallopian adj3 adhesion\$).tw. (12)

12 (fallopian\$ adj3 block\$).tw. (30)

13 (fallopian adj3 adhesion\$).tw. (12)

14 hydrosalpin\$.tw. (505)

15 (surg\$ adj5 tub\$).tw. (3324)

16 (surg\$ adj5 fallopian\$).tw. (121)

17 salping\$.tw. (4367)

18 (aspirat\$ adj5 hydrosalpin\$).tw. (12)

19 (clip\$ adj5 hydrosalpin\$).tw. (1)

20 essure\$.tw. (64)

21 exp uterine tube disease/ or exp hydrosalpinx/ or exp salpingitis/ or exp tuboovarian abscess/ or exp uterine tube abscess/ or exp uterine tube occlusion/ (4002)

22 or/6-21 (16567)

2322 and $5(1137)$

24 Controlled study/ or randomised controlled trial/ (2734301)

25 double blind procedure/ (70094)

26 single blind procedure/ (7722)

27 crossover procedure/ (20517)

28 drug comparison/ (81252)

29 placebo/ (116586)

30 random\$.ti,ab,hw,tn,mf. (415876)

31 latin square.ti,ab,hw,tn, mf. (1107)

32 crossover.ti,ab,hw,tn,mf. (35368)

33 cross-over.ti,ab,hw,tn,mf. (11959)

34 placebo\$.ti,ab,hw,tn,mf. (167040)

35 ((doubl\$ or singl\$ or tripl\$ or trebl\$) adj5 (blind\$ or mask\$)).ti,ab,hw,tn, mf. (115237)

36 (comparative adj5 trial\$).ti,ab,hw,tn,mf. (15269)

37 (clinical adj5 trial\$).ti,ab,hw,tn,mf. (576643)

38 or/24-37 (3243356)

39 nonhuman/ (3097648)

40 animal/ not (human/ and animal/) (14472)

41 or/39-40 (3101342)

4238 not $41(1915335)$

$43 \quad 42$ and 23 (297)

44 limit 43 to $y r=" 2006$ - 2008" (61) 
45 from 44 keep 1-61 (61)

\section{Database: CINAHL - Cumulative Index to Nursing \& Allied Health}

1 (in vitro fertilisation or in vitro fertilization).tw. (376)

2 (ivf or icsi).tw. (316)

3 intracytoplasmic sperm injections.tw. (0)

4 exp reproductive techniques, assisted/ or exp embryo transfer/ or exp fertilization in vitro/ or exp

sperm injections, intracytoplasmic/ or exp zygote intrafallopian transfer/ (1002)

5 (ART or embryo transfer or et).tw. (45441)

6 or/1-5 (46463)

7 exp Fallopian Tube Diseases/ (152)

8 Fallopian Tube Disease\$.tw. (1)

9 salping\$.tw. (134)

10 (fallopian adj3 disease\$).tw. (1)

11 (tub\$ adj5 adhesion\$).tw. (9)

12 (tub\$ adj3 occlusion\$).tw. (63)

13 (tub\$ adj5 block\$).tw. (53)

14 hydrosalpin\$.tw. (13)

15 (surg\$ adj5 tub\$).tw. (184)

16 (aspirat\$ adj5 hydrosalpin\$).tw. (0)

17 essure\$.tw. (25)

18 (clip\$ adj5 hydrosalpin\$).tw. (0)

19 hysteroscop\$.tw. (172)

20 or/7-19 (736)

216 and 20 (37)

22 exp clinical trials/ (61758)

23 Clinical trial.pt. (32434)

24 (clinic\$ adj trial\$1).tw. (14019)

25 ((singl\$ or doubl\$ or trebl\$ or tripl\$) adj (blind\$3 or mask\$3)).tw. (8276)

26 Randomi?ed control\$ trial\$.tw. (12024)

27 Random assignment/ (18434)

28 Random\$ allocat\$.tw. (1272)

29 Placebo\$.tw. (11470)

30 Placebos/ (4474)

31 Quantitative studies/ (4044)

32 Allocat\$ random\$.tw. (74)

33 or/22-32 (85043)

3433 and 21 (4)

35 from 34 keep 1-4 (4)

\section{Appendix 2. Items of data extraction}

The following characteristics were assessed:

(1) Trial characteristics

(a) Study design:

(i) Randomised controlled trial

(ii) Patient recruitement.

(iii) Patient sampling

(iv) Patient in- and exclusion criteria

(v) Duration of follow-up

(vi) Type of follow-up

(c) Size of study:

(i) Number of women recruited

(ii) Number of women randomised

(iii) Number of women excluded

(iv) Number of women withdrawn and lost to follow-up

(v) Number of women analysed

(d) Study setting:

(i) Single-centre or multicentre

(ii) Location 
(iii) Timing and duration

(e) Criteria for surgical treatment prior to IVF:

(i) Tubal disease

(ii) Hydrosalpinx

(iii) Either of the above plus previous failed IVF

(2) Characteristics of the study participants

(a) Baseline characteristics

(i) Age

(ii) Primary or secondary infertility

(iii) Duration of infertility

(iv) Investigative work-up - baseline follicle-stimulating hormone (FSH), semen analysis, diagnosis of tubal pathology, confirmatory test of ovulation

(v) Other contributory causes to infertility than tubal disease

(vi) Previous treatments - IVF and other treatments

(b) Treatment characteristics

(i) IVF protocol

(ii) Time from surgery to IVF

(iii) Proportion undergoing intracytoplasmic sperm injection (ICSI)

(iv) Number of oocytes retrieved at IVF

(iii) Fertilisation rate

(iv) Number of embryos transferred

(3) Interventions

(a) Timing of surgical intervention

(b) Nature of surgical intervention

(c) Absence of other interventions in treatment and control group

(4) Outcomes

(a) Primary

(i) Live birth rate

(b) Secondary

(i) Ongoing pregnancy rate

(ii) Viable pregnancy rate

(iii) Clinical pregnancy rate

(iv) Biochemical pregnancy rate

(v) Ectopic pregnancy rate

(vi) Miscarriage rate

(vii) Multiple pregnancy rate

(viii) Surgical complication rate

(ix) Ovarian response to IVF

\section{Appendix 3. Assessment of risk of bias}

Risk of the following bias were assessed, according to the following terms:

(1) Studysize bias - was a power calculation performed and adhered to?

(2) Selection bias - was allocation concealment was not performed by any of the following methods: central computer randomisation, on site assignment, or sealed opaque envelopes?

(3) Detection bias - were both the physician performing IVF and the outcome assessor blinded? And was the length of follow-up was adequate to detect the stated outcome measure(s)? Blinding of patient blinding was not applied as a quality criterion; as this sham tubal surgical intervention is not ethical and less relevant as patients cannot influence the occurrence or the detection of pregnancy.

(4) Attrition bias - was loss to follow-up of patients accounted for and dealt with an intention-to-treat analysis?

(5) Selective reporting bias -were there no suggestions of selective reporting?

(7) Funding bias - Was there no source of funding of the trial, or was this stated?

All quality items were scored with yes (suggesting the absence or a low risk of bias), no or unclear (suggesting a risks of bias of the certain item).

WHAT'S NEW 


\begin{tabular}{|c|c|c|}
\hline Date & Event & Description \\
\hline 25 May 2008 & $\begin{array}{l}\text { New citation required but conclusions } \\
\text { have not changed }\end{array}$ & $\begin{array}{l}\text { This review was updated in August 2008. New authors have } \\
\text { joined the team: SVV, AS and BWJM. As compared to 2004; study } \\
\text { identification and selection was performed by a second review- } \\
\text { er based upon a slightly adjusted search. Three new trials were } \\
\text { included: Kontoravdis 2006; Moshin 2006;and Hammadieh } 2008 \text {. } \\
\text { Therefore this review now includes studies reporting the ef- } \\
\text { fect of ultrasound guided aspiration of hydrosalpinges and the } \\
\text { efficacy of tubal occlusion. A previously included trial was ex- } \\
\text { cluded: Goldstein } 1998 \text {. One ongoing study was found: Darwish } \\
\text { 2005,2006, 2007; this preliminary study assessed the feasibility } \\
\text { of hysteroscopic tubal occlusion with electrocautery. Follow-up } \\
\text { time was not long enough to assess results of pregnancy. Data } \\
\text { extraction and data analysis of the three newly included studies } \\
\text { was completed by the second reviewer (SVV) in consensus with } \\
\text { the first reviewer. Outcomes were extracted according to stricter } \\
\text { definitions of Live birth, ongoing pregnancy, viable-clinical or } \\
\text { biochemical pregnancy. Results were imported in Revman } 5 \text { and } \\
\text { analysis was undertaken. The Cochrane manuscript was rewrit- } \\
\text { ten and edited by the same panel and two newly added authors: } \\
\text { B.W. Mol and A. Strandell. }\end{array}$ \\
\hline
\end{tabular}

\section{H I S T O R Y}

Protocol first published: Issue 2, 2000

Review first published: Issue 3, 2001

\begin{tabular}{lll}
\hline Date & Event & Description \\
\hline 29 April 2008 & $\begin{array}{l}\text { New citation required and conclusions } \\
\text { have changed }\end{array}$ & Substantive amendment \\
\hline
\end{tabular}

\section{CONTRIBUTIONSOF AUTHORS}

Neil Johnson conceptualised the protocol and the review primarily, and carried out the search, selection of trials and risk of bias assessment as a first reviewer and first author in the first version of the review and as a second reviewer in the current update.

Sabine van Voorst conducted the current update by performing the search and selection of trials, risk of bias assessment as a first reviewer and author. Martin Sowter proofread the primary protocol, was available to resolve discrepancies for differences of opinion between the other authors and added content expertise to the discussion and conclusions during the first and updated version of this review. Ben Willem Mol and Annika Strandell proofread the updated review and added content expertise to the discussion and conclusion.

\section{DECLARATIONSOF INTEREST}

Two of the authors (MS and AS) have performed clinical studies assessing the interventions studied in this review (Sowter 1997; Strandell 1999), the latter being one of the included RCTs in this review.

Neil Johnson works as a gynaecologist at Auckland City Hospital (a public hospital) in the National Women's Minimal Access Surgery and Endometriosis Service. NJ is also a private gynaecologist with groups called Endometriosis Auckland and Repromed Auckland. Within the last 3 years NJ has received financial support to attend conferences or to arrange research meetings from the following companies: Organon, Serono, Schering and Device Technologies.

Sabine van Voorst at the time was a medical student of the faculty of Health, Medicine and Life Sciences of the University of Maastricht, the Netherlands. She is now a resident in Obstetrics and Gynaecology at the Reinier de Graaf Gasthuis in Delft, the Netherlands. She has no financial conflict of interest. 
Annika Strandell is a gynaecologist at Kungälv Hospital, associate professor at the University of Gothenburg and employed at the regional center for Health Technology Assessment in Göteborg, Sweden. She was the principle investigator and co-ordinator of the Scandinavian trial on salpingectomy for hydrosalpinges prior to IVF. She has no financial conflict of interest.

\section{SOURCES OF SUPPORT}

\section{Internal sources}

- University of Auckland, School of Medicine, Auckland, New Zealand.

\section{External sources}

- No sources of support supplied

\section{DIFFERENCES BETWEEN PROTOCOLANDREVIEW}

The current updated review differs from the original protocol in primary outcomes and risk of bias assessment. Definitions of fertility outcomes are applied stricter as of the 2008 update, reflecting current thoughts on fertility outcome (Dias 2006; Clarke 2008). Current risk of bias assessment reflects the current opinion in assessing quality of studies (Higgins 2008).

\section{N DEX TERMS}

\section{Medical Subject Headings (MeSH)}

${ }^{\star}$ Fertilization in Vitro; Fallopian Tube Diseases [ ${ }^{\star}$ surgery]; Fallopian Tubes [*surgery]; Pregnancy Outcome; Randomized Controlled Trials as Topic

\section{MeSH check words}

Female; Humans; Pregnancy 